LA-8830-MS

\title{
MASTER
}

Nucleonic Analysis of the

ETF Neutral-Beam-Injector-Duct and Vacuum-Pumping-Duct Shields 


\title{
LA-8830-MS
}

UC-20d

Issued: May 1981

\section{Nucleonic Analysis of the \\ ETF Neutral-Beam-Injector-Duct and \\ Vacuum-Pumping-Duct Shields}

\author{
W. T. Urban \\ T. J. Seed \\ Donald J. Dudziak
}

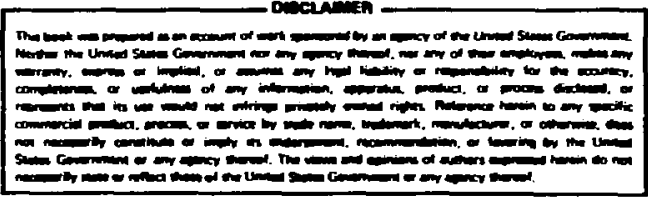




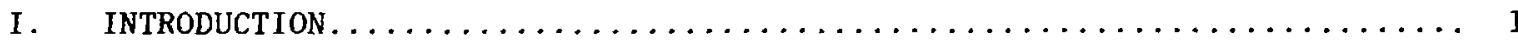

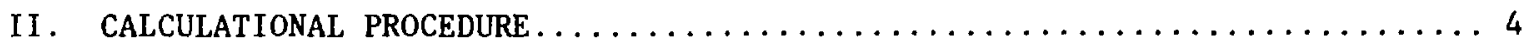

II I. MONTE CARLO MODELS AND CALCULATIONS $\ldots \ldots \ldots \ldots \ldots \ldots \ldots \ldots \ldots \ldots \ldots \ldots$

A. Torus Calculations $\ldots \ldots \ldots \ldots \ldots \ldots \ldots \ldots \ldots \ldots \ldots \ldots \ldots \ldots \ldots \ldots$

B. NBI-Duct Wall Surface Source Calculations ................. 16

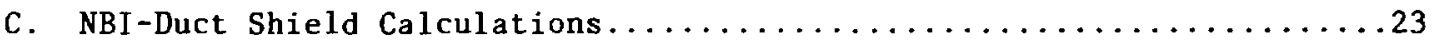

D. VP-Duct Wall Surface Source Calculations..................25

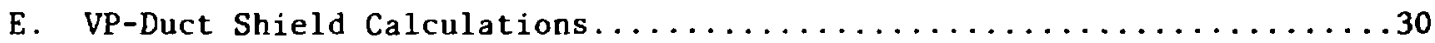

IV. TWO-DIMENSIONAL DISCRETE ORDINATES (TRIDENT-CTR) CALCULATIONS ........32

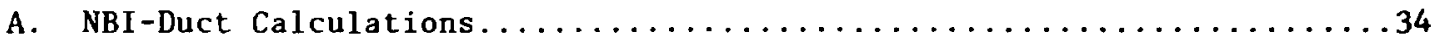

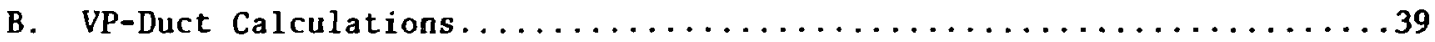

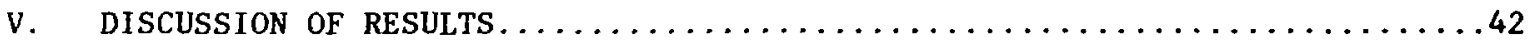

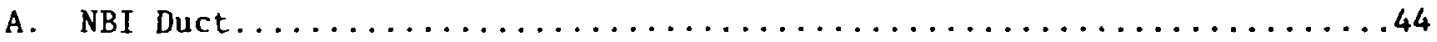

1. MCNP Results...........................44

2. TRIDENT-CTR Results.......................... 50

3. Comparison of MCNP and TRIDENT-CTR................. 59

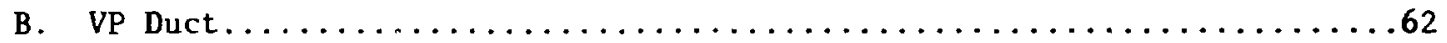

1. MCNP Results..............................62

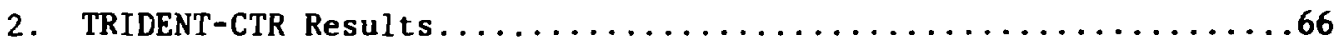

3. Comparison of MCNP and TRIDENT-CTR Results.............71

C. Comments on Accuracy.......................... $\ldots \ldots$

VI. CONCLUSTONS $\ldots \ldots \ldots \ldots \ldots \ldots \ldots \ldots \ldots \ldots \ldots \ldots \ldots \ldots \ldots \ldots \ldots \ldots$

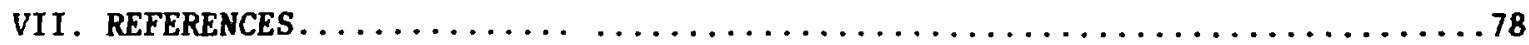


NUCLEONIC ANALYSIS OF THE ETF NEUTRAL-BEAM-INJECTOR-DUCT

AND VACUUM-PUMPING-DUCT SHIELDS

by

W. T. Urban

T. J. Seed

Donald J. Dudziak

\begin{abstract}
A nucleonic analysis of the Engineering Test Facility neutral-beam-injector-duct and vacuum-pumping-duct shields has been made using a hybrid Monte Carlo/discrete-ordinates method. This method used Monte Carlo to determine internal and external boundary surface sources for subsequent discrete-ordinates calculations of the neutron and gamma-ray transport through the shields. Confidence was provided in both the $h^{\text {.h }}$. the results obtained through a comparison with three-dimensional Monte Carlo results. Also determined in the analysis were the energy and angular distributions of neutrons and gamma rays entering the neutral-beam-injector duct from the toroidal plasma chamber, as well as exiting the duct into the neutral-beaminjector chamber. In addition, the energy and angular distributions of neutrons entering the vacuum-pumping chamber were determined.
\end{abstract}

\title{
I. INTRODUCTION
}

The Engineering Test Facility (ETF) conceptual shielding for the neutra1beam-injector (NBI) and the vacuum-pumping (VP) ducts has been analyzed through the marriage of three-dimensional (3-D) Monte Carlo calculations with two-dimensional (2-D) discrete-ordinates calculations. Figure 1 illustrates schematically 

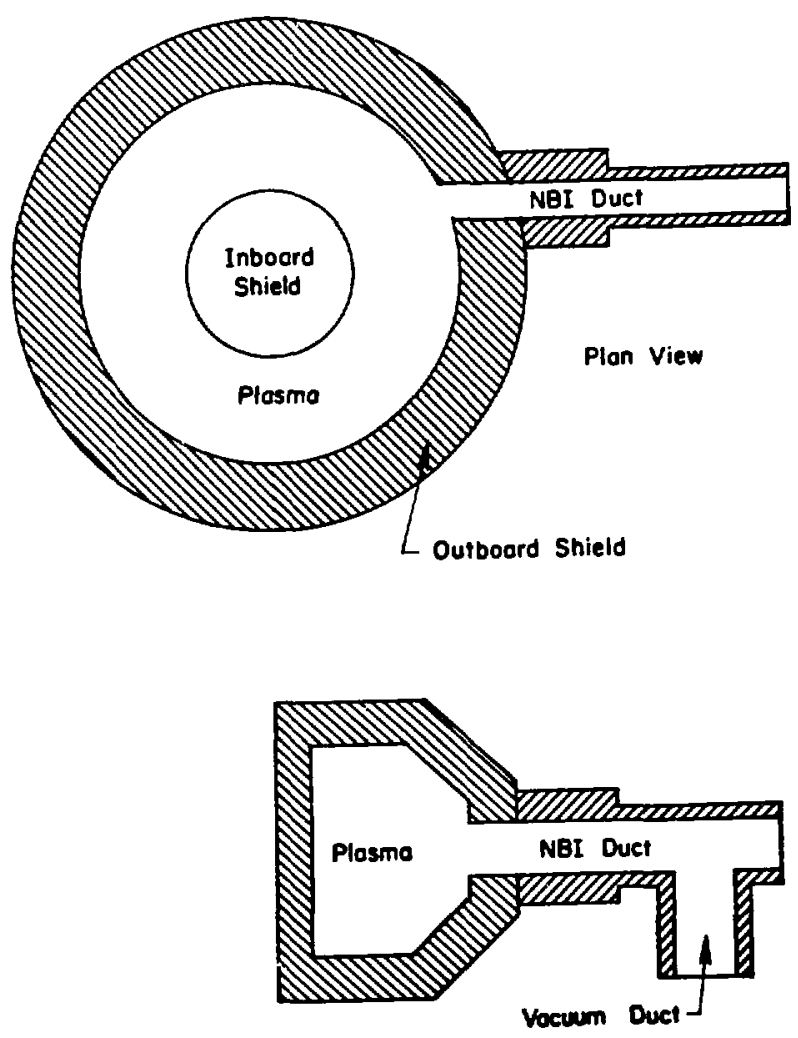

Elevation

Fig. 1. Schematic of the ETF geometry considered for the MCNP Monte Carlo calculations (not to scale).

the geometrical relationships of these ducts to the ETF torus and to one another. Presented herein is a description of the method used and the results obtained in the analysis of the NBI and VP ducts and their respective shielding.

A primary objective of this effort was to provide data with which the NBiand VP-duct shields could be evaluated in terms of the ETF design criteria. Even though the shield designs considered were only conceptual, the results of this analysis provide hard information upon which subsequent shield designs can be evaluated. Furthermore, a secondary benefit has been the determination of the radiation flow, both neutron and gamma ray, into the NBI and VP chambers. This information may well be of greater importance than the analysis of the duct shields, as it can be used to evaluate radiation effects in these chambers. In particular, it can provide the starting point for assessing the implications of these radiation effects and how they impact on the viability of the NBI configuration itself. 
Evaluation of the NBI and VP ducts and shielding requires first that an accurate knowledge of the neutrons and gamma rays entering the NBI duct be available. For this reason the geometry considered must include the toroidal plasma chamber and its associated inboard and outboard shields, as well as the two jucts and their shielding. Furthermore, it is recognized that the shield thicknesses, compositions, and sizes of the ducts are conceptual at this stage of the design and subject to change. The parameters used in this analysis reppresent the best available at the initiation of this effort, at which time they were frozen.

The analysis of large ducts and their associated shielding is consistently a problem. $^{1}$ Although deterministic methods such as discrete ordinates can easily provide the desired pointwise responses at deep penetrations in a shield, they are hampered by streaming effects associated with neutron transport in a vacuum, and their geometry limitation. On the other hand, Monte Carlo methods can readily handle $3-\mathrm{D}$ geometries and streaming down ducts but cannot reasonably provide pointwise responses over an entire shield. The approach taken here has been to marry the two methods in such a manner as to take advantage of the strong points of each method.

Conceptually, the application of a hybrid Monte Carlo/discrete-ordinates method is straightforward. Simply stated, Monte Carlo was used to determine spatial, angular, and energy - dependent source distributions of neutrons and gamma rays incident on the duct walls and appropriate external boundaries. The incident flow of radiation was then transformed into surface sources for use in 2-D discrete-ordinates calculations to obtain spatially dependent fluxes throughout the shields.

Three-dimensional calculations were performed using the continuous-energy Monte Carlo code MCNP, ${ }^{2}$ and 2-D calculations were performed using the triangularmesh discrete-ordinates code TRIDENT-CTR. ${ }^{3}$ Both codes are available to the fusion community through either the Radiation Shielding Information Center (RSIC) or the National Magnetic Fusion Energy Computer Center (NMFCC).

An attempt was specifically made to use only computer codes and nuclear data available to the entire fusion nucleonics community by RSIC or the NMFCC. Thus, the calculational method should be reproducible and generally available to shield designers. The only exception to achieving this objective was in the processor code required to link the MCNP and TRIDENT-CTR calculations by a surface source. 
It has been an objective in this work to extract as much useful information from the calculacions as possible. Thus, some of the data presented may appear extraneous relative to the primary objectives stated at the beginning of this section. However, this information has been included because few calculations of this complex a configuration are performed with the rigor used in this work, and such data may prove useful to fusion reactor shield designers for "back-ofthe-envelope" or scaling calculations. It was an objective to obtain Monte Carlo results with relative errors of less than $10 \%$. However, also reported are some results that have errors greater than $10 \%$, with the understanding that the larger their error the less statistically reliable are the results. This reduction in reliability results because the variance or the variance increases and, therefore, a small calculated error is possible even when the answer is far from converged. Such information is provided in lieu of anything better, bui should be used with extreme caution. Finally, reference to TRIDENT-CTR results in this report is equivalent to hybrid MCNP/TRIDENT-CTR results because all TRIDENTCTR calculations employ surface sources determined by MCNP.

Organization of this report is as follows. Section II contains an overview of the ETF configuration and the calculational procedure. Sections III and IV, respectively, contain descriptions of the models and calculations for MCNP and TRIDENT-CTR. Section III presents some intermediate calculational results, e g. MCNP torus results. However, Sec. $V$ is where the majority of the results are presented, discussed, and compared. Section $V$ also contains a briaf aiscussion of the accuracy of the results. Sections VI and VII contain conclusions and references, respectively.

\section{CALCULATIONAL PRCCEDURE}

The configuration of Fig. 1 was broken into three distinct geometrical segments to facilitate acquisition of the required information and to minimize the duplication of calculational effort. Segmentation of the problem was accomplished, from a calculational vievpoint, through the use of trapping surfaces in the MCNP calculations. When a particle crosses a trapping surface, the spatial coordinates, direction cosines, energy, weight, and time are written to a file. The Monte Carlo calculation can then be continued with the "trapped" particle information being used to define a secondary source plane. Trapping 
surfaces were located at the torus outboard shield inner radius/NBI-duct mouth interface, and at the NBI-duct/VP-duct mouth interface. This procedure not only allows the problem to be broken into more tractably sized geometries but also allows one to go back and rerun one part of the problem that is slightly perturbed without redoing the entire calculation starting from the torus. For example, a slightly different VP-duct shield could be analyzed starting with the trapping surface source at the NBI-duct/VP-duct mouth interface.

Segmentation of the configuration is required for the $(r, z)$-geometry TRIDENT-CTR calculations. The NBI duct is considered as one problem and the VP duct as the other. The TRIDENT-CTR calculations are independent of the way in which the problem is modeled with MCNP as long as suitable surface sources are obtained.

Because of the hybrid MCNP/TRIDENT-CTR approach used and the segmentation of the problem, a number of calculational steps were undertaken in this analysis. In order to provide an overview for the more detailed discussions that follow, these steps are listed below.

1. An MCNP slculation was made using only the torus geometry, including the inboard and outboard shields as well as the duct penetration through the outboard shield. From this calculation the energy- and angular-dependent neutron and gamma-ray flows, both entering the NBI duct and incident on the outboard shield first wall adjacent to the NBI duct, were obtained.

2. Using the results from step 1 to define a surface source at the NBI duct mouth, an MCNP calculation was performed to determine the spatially dependent energy and angular distribution of :eutrons and gamna rays incident on the duct walls. Because no transyort in the duct shielding was considered, this calculation was essentially one of ray tracing.

3. The neutron and gamma-ray flow incident on the torus outboard shield from step 1 and that incident on the duct walls from step 2, were used to generate boundary and internal surface sources for an $(r, z)$ geometry TRIDENT-CTR calculation, from which the neutron and gamma-ray flux distributions throughout the NBI shield were obtained.

4. Using th. Elow of neutrons and gamma rays incident on the torus outboard shield and entering the NBI duct from step 1, MCNP calculations 
were made to obtain the neutron flux in the duct and also selected responses in the NBI shielding for comparison with TRIDENT-CTR results from step 3. This calculation also provides, by a trapping surface, the energy- and angular-dependent neutron and gamma-ray flow entering the VP duct and incident on the NBI-duct vall adjacent to the NBIduct/VP-duct interface.

5. Results from step 4 were used to define a surface source at the VP-duct mouth that was incorporated into an MCNP calculation to determine the spatially dependent energy and angular distribution of neutrons and gamma rays incident on the VP-duct walls. This calculation was essentially one of ray tracing as no transport was allowed in the VP-duct shielding.

6. The neutron and gamma-ray flow incident on the NBI duct wall adjacent to the NBI-duct/VP-duct interface from step 4, and that on the VP-duct walls from step 5, were used to generate boundary and internal surface sources for an $(r, z)$-geometry, TRIDENT-CTR calculation to obtain the neutron and gamma-ray flux distribution throughout the VP-duct shield.

7. Using the flow of neutrons and gamma rays incident on the NBI-duct wall adjacent to the NBI-duct/VP-duct interface and entering the VP duct frum step 4, MCNP calculations were made to obtain the neutron flux in the duct and also selected responses in the VP-duct shielding for comparison with the TRIDENT-CTR results from step 6.

\section{MONTE CARLO MODELS AND CALCULATIONS}

Monte Carlo calculations were made using the three distinct geometrical segments of the overall problem as described at the beginning of section II. The purpose of these calculations was two-fold: (1) to obtain data from which surface sources for use with TRIDENT-CTR could be constructed, and (2) to allow an intercomparison of Monte Carlo and discrete-ordinates results at sclected locations in the ducts and their shields. A spin-off of these calculations was that detailed information was obtained relating to the radiation entering, traversing, and exiting each duct.

Because of the way in which the problem was approached, five different Monte Carlo models were required, one of which was solely for the purpose of 
benchmarking the TRIDENT-CTR results. These consist of one torus model and two models each for the NBI and VP ducts. Calculational continuity between the geometrical segments was maintained through the use of trapping surfaces at the interfaces between the segments. The sequence of these calculations and the use of trapping surfaces was described in Section II.

The MCNP Monte Carlo calculations used cross sections from the MCNP Recommended Monte Carlo Cross-Section (RMCCS) Library. ${ }^{2,4}$ Editing of the results made use of energy breakpoints consistent with a standard Los Alamos 42 energy-group structure that contains 30 neutron and 12 gamma-ray energy groups. ${ }^{5}$ This energy-group structure is presented in Tabie I.

Materials used in the MCNP calculations were carbon armor, stainless steel, and a homogeneous mixture of stainless steel and borated water. The homogeneous mixture consisted of 70 vol\% stainless steel and 30 vol\% borated water $(0.4$ at.\% ${ }^{10} \mathrm{~B}$ ). Isotojic compositions of these three materials are contained in Table II.

\section{A. Torus Calculations}

The torus model contained both the inboard and outboard shields, and an effort was made to minimize geometrical approximations of either of these shields or the plasma geometry. Figures 2 and 3 are elevation and plan views, respectively, of the torus MCNP model. The outboard shield consisted of $0.3 \mathrm{~m}$ of stainless steel followed by $0.9 \mathrm{~m}$ of the homogeneor:s mixture of stainless steel and borated water. The inboard shield consisted of $0.02 \mathrm{~m}$ of carbon armor, $0.205 \mathrm{~m}$ of stainless steel and $0.615 \mathrm{~m}$ of the homogereous mixture of stainless steel and borated water.

The plasma was represented as a uniformly distributed source of 2.25-pJ (14-MeV) neutrons which are produced with an isotropic angular distribution. This source was D-shaped in cross section with its axis of revolution congruent with the toroidal axis. Normalization of the source was such as to represent a volumetric source strength of $1.38 \times 10^{18}$ plasma neutrons $/ \mathrm{m}^{3} \mathrm{~s}$. This source strength corresponds to a plasma volume of $290 \mathrm{~m}^{3}$ and a power of $1130 \mathrm{MW}$ (at $17.6 \mathrm{MeV} /$ reaction). This normalization results in an incident neutron-energy flow rate on the outboard shield due to neutrons with energies between 13.5 and $15.0 \mathrm{MeV}$ of $\sim 2.4 \mathrm{MW} / \mathrm{m}^{2}$. 
TABLE I

30/12 ENERGY-GROUP STRUCTURE

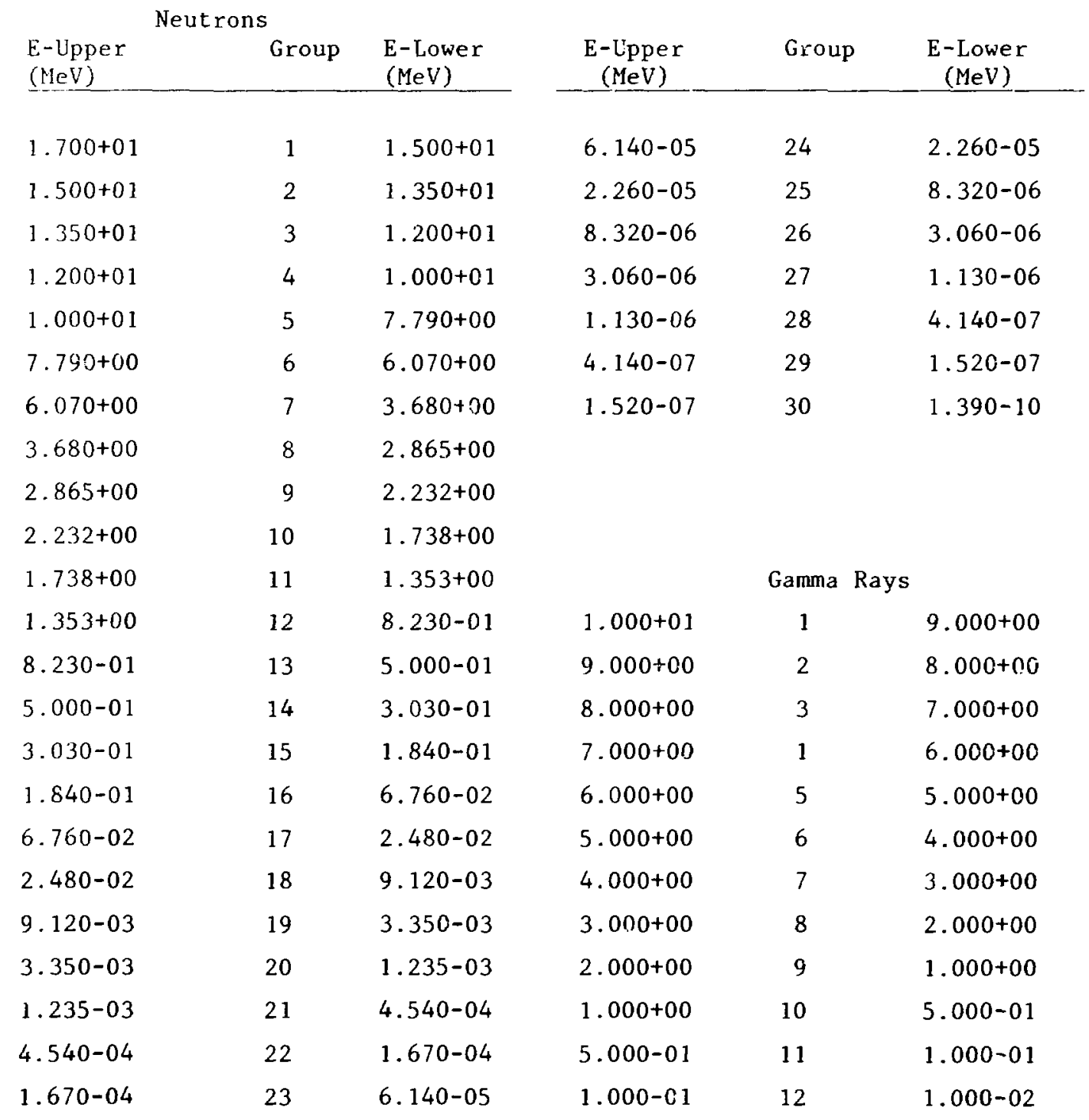


TABLE II

ATOM DENSITIES OF MATERIALS

Element
$\mathrm{Ni}$
$\mathrm{Cr}$
$\mathrm{Fe}$
$\mathrm{Mn}$
$\mathrm{Mo}$

Atoms $/ \mathrm{m}^{3}$

Stainless Steel
$1.15+28^{\mathrm{a}}$
$1.67+28$
$5.44+28$
$1.75+27$
$1.51+27$

Stainless Sceel-Borated Water Mixture ${ }^{b}$

$\mathrm{Ni}$
$\mathrm{Cr}$
$\mathrm{Fe}$
$\mathrm{Mn}$
$\mathrm{Mo}$
$\mathrm{O}$
$\mathrm{B}-10$
$\mathrm{H}$

Carbon Armor

c
$8.050+27$
$1.169+28$
$3.808+28$
$1.225+27$
$1.057+27$
$1.005+28$
$1.200+26$
$2.010+28$

$8.030+28$

$a_{1.15}+28=1.15 \times 10^{28}$.

$\mathrm{b}_{70}$ vol\% stainless steel and 30 vol\% borated $\left(0.4\right.$ atom/o $\left.{ }^{10} \mathrm{~B}\right)$ water. 


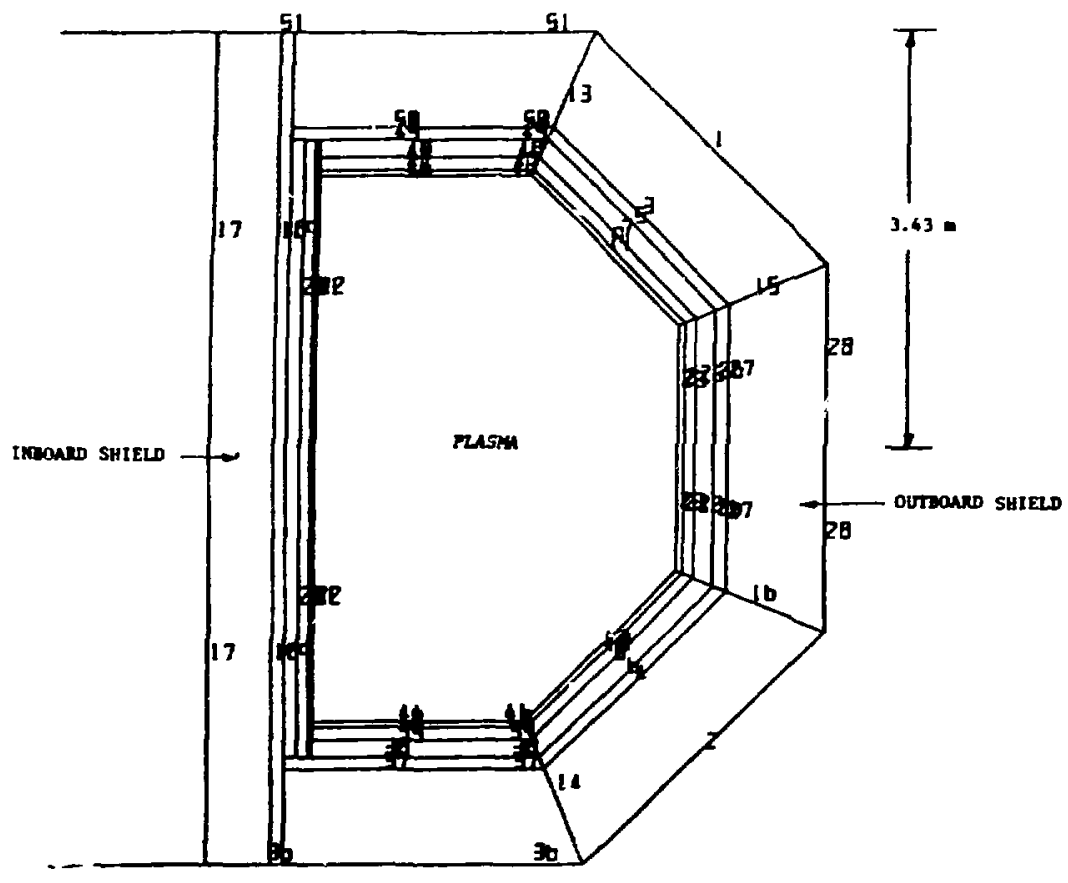

Fig. 2. Elevation cross section through the torus at a toroidal location not including NBT duct; MCNP model (numbers on figure are surface numbess from MCNP plotting routine).

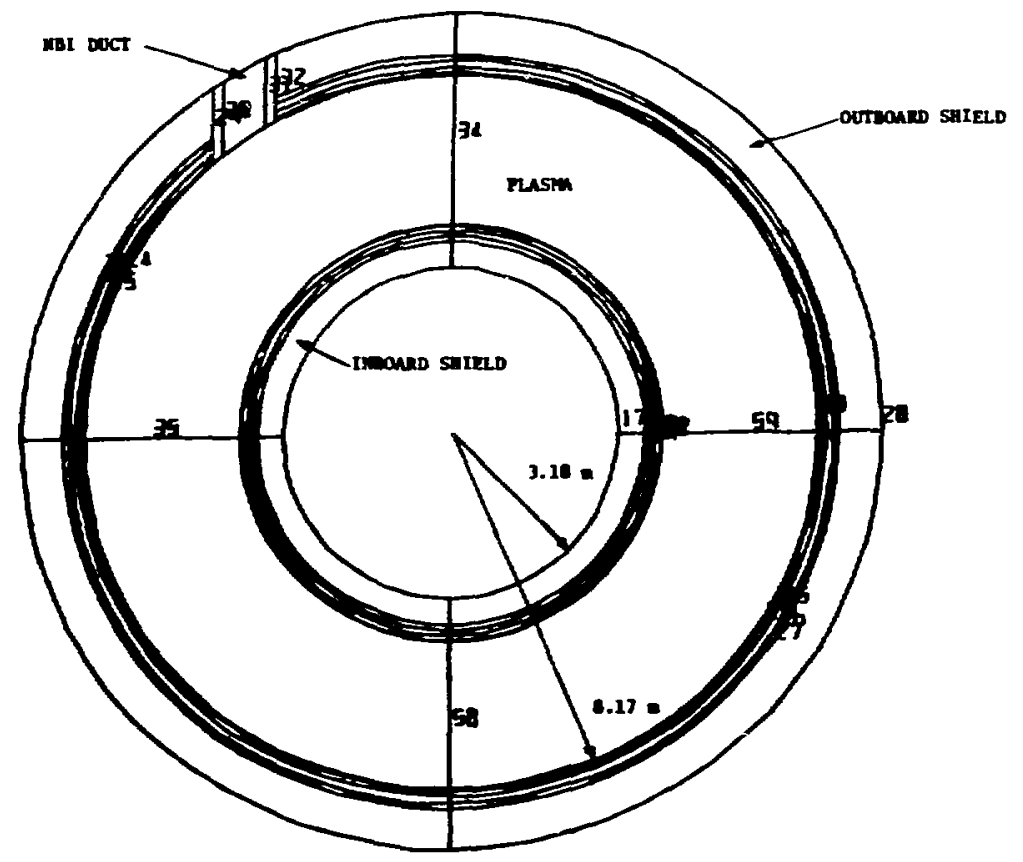

Fig. 3. Plan view at the torus midplane; MCNP model. 
A rectangular trapping surface with sides approximately $0.5 \mathrm{~m}$ greater than the actual NBI-duct opening and whose center was congruent with the duct center was used at the torus outboard shield inner radius/NBI-duct mouth interface. Use of the larger trapping surface allows for including lip penetration contributions to neutron and gamma-ray results in subsequent NBI-duct calculations. The efficiency of the trapping surface was increased by trapping all particles crossing the outboard shield inner radius in a positive (outboard) direction over the axial extent of the trapping surface. These particle crossings were rotated, at a constant axial height, around the torus to the trapping-surface location where they were uniformly distributed. Furthermore, the direction cosines were rotated to preserve the particle direction relative to the outboard shield inner radius, and the particle weights were adjusted to reflect this variance reduction procedure.

MCNP was run in the coupled neutron/gamma-ray mode. A total of 120000 source neutrons were started, which resulted in 122360 trapped neutrons and 19565 trapped gamma rays. These trapped neutrons and gamma rays provide the basis for continuing the calculation into the NBI duct. The results of this intermediate calculation (i.e., the torus calculation) are presented below.

Here, as well as throughout this report, Monte Carlo results are often reported in terms of neutron or gamma-ray flow. Flow is simply defined to be the number of particles per unit area per unit time incident on (i.e., crossing) a surface in either a positive or negative direction with respect to the surface in a given arbitrary angular bin.

The energy distributions of the neutron and gamma-ray flow across the trapping surface in a positive direction (i.e., from the plasma chamber into the ouboard shield or NBI duct) are presented in Figs. 4 and 5, respectively. Approximately $25 \%$ of the neutrons have energies between 13.5 and $15.0 \mathrm{MeV}$ and approximately $69 \%$ have energies below $1.353 \mathrm{MeV}$. Approximately $67 \%$ of all gamma rays have energies below $1.0 \mathrm{MeV}$. Neutron spectral data have fractional errors (i.e., relative standard deviations) ranging from approximately 0.8 to $7.5 \%$. The value of the normalized total neutron flow is $4.14 \times 10^{14}$ neutrons $/ \mathrm{cm}^{2} \mathrm{~s}$ and its fractional error is $0.5 \%$. Gamma-ray spectral data errors vary from 2 to $14 \%$ and the normalized total gamma-ray flow is $1.05 \times 10^{14}$ gamma rays $/ \mathrm{cm}^{2} \mathrm{~s}$ with an error of $1.0 \%$. Fractional errors, in terms of a percentage, quated above and throughout this report are at the $68 \%$ confidence level. 


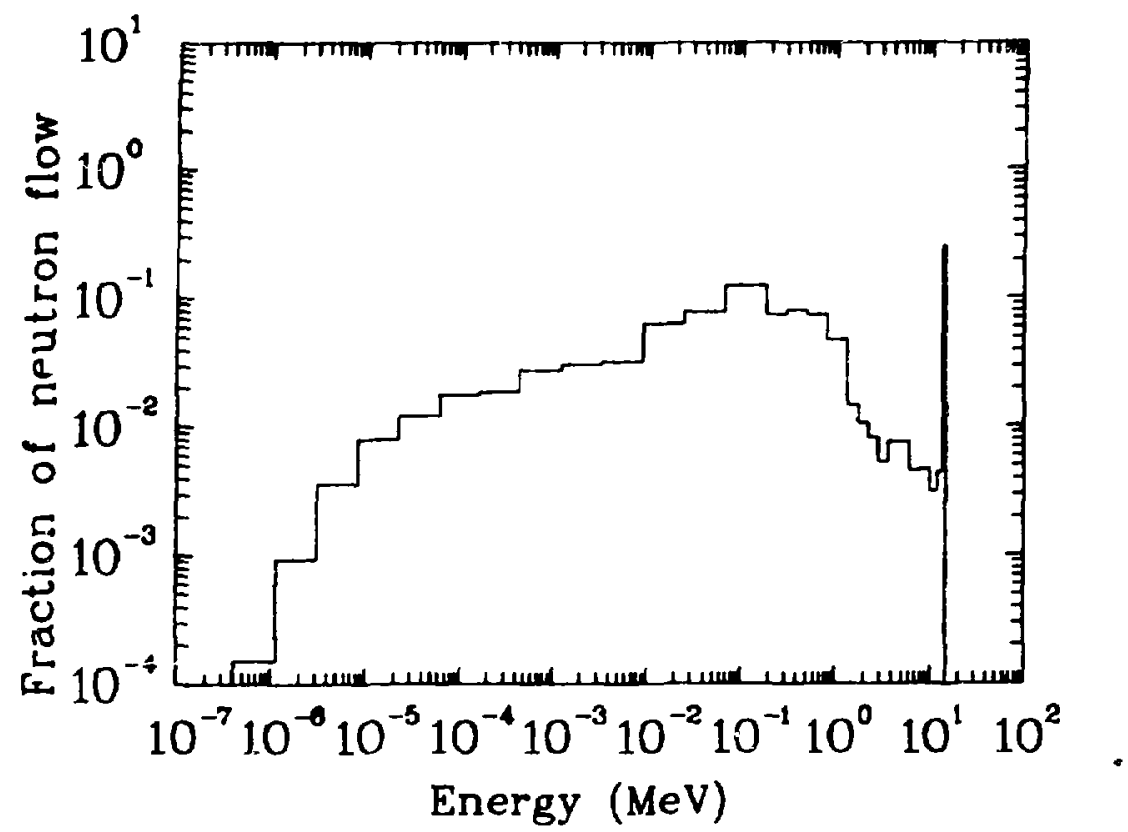

Fig. 4. Neutron flow in the outboard direction at the torus outboard shield, at and around the NBI duct mouth. Neutron flow normalized to one neutron. (MCNP results.)

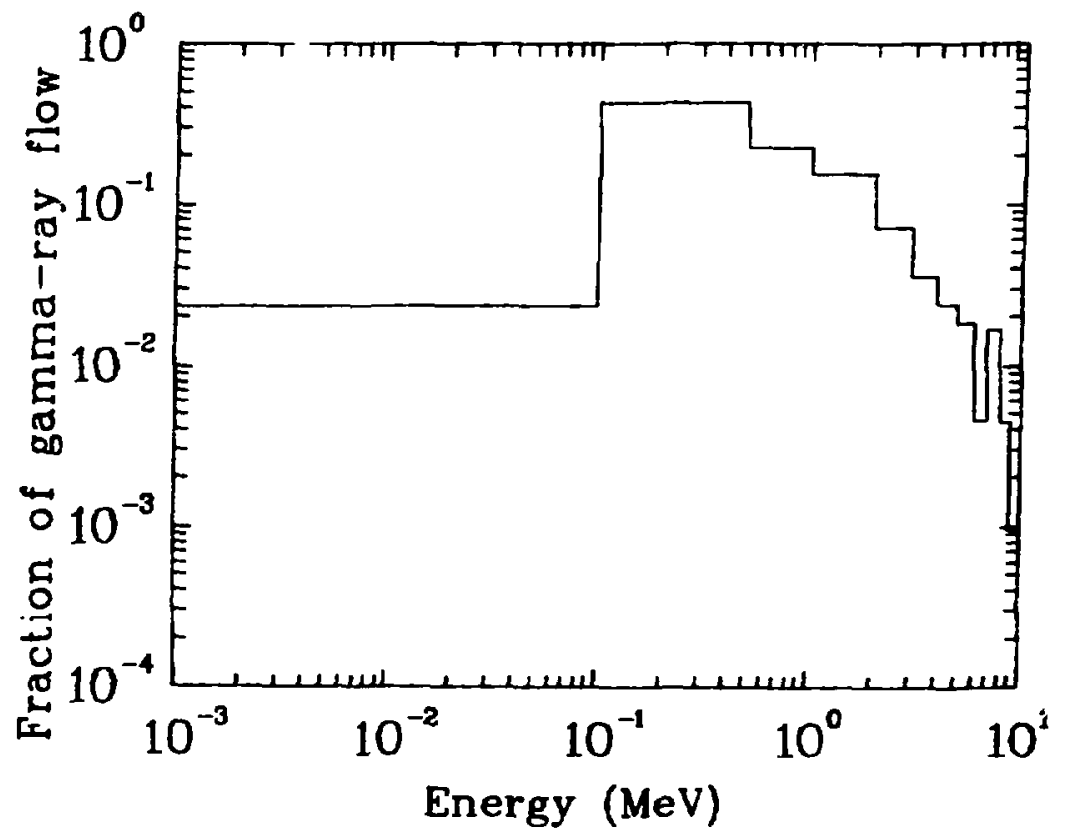

Fig. 5. Gamma-ray flow, in the outboard direction, across the torus outboard shield first wall, at and around the NBI duct mouth. Flow normalized to one gama ray. (MCNP results.) 
The angular distribution of the neutron flow across the trapping surface in the positive direction is shown in Fig. 6 for two energy groups; less than $13.5 \mathrm{MeV}$ and greater than $13.5 \mathrm{MeV}$. Each curve has been normalized to one incident neutron in order to more readily allow comparison of the two histograms. The abscissa, labeled cosine(theta), represents the cosine of the angle that the particle track makes with the outward normal to the trapping surface. Examination of the angular distribution on a more detailed energy structure revealed that the angular distribution of energy groups below $13.5 \mathrm{MeV}$ could be well approximated by the average over-all groups below $13.5 \mathrm{MeV}$. Furthermore, it was found that the angular distribution for neutrons below $13.5 \mathrm{MeV}$ was a good approximation of the gamma-ray angular distribution shown in Fig. 7 . This latter result is not surprising in that it is the low-energy neutrons that give rise to the gamma rays through interactions in the inboard and outboard shields. Figure 8 presents the ratio of these two angular distributions, i.e., gamma - ray to neutron (less than $13.5 \mathrm{MeV}$ ). At all values of cosine (theta) the ratio is within $10 \%$ of unity. The angular distributions of

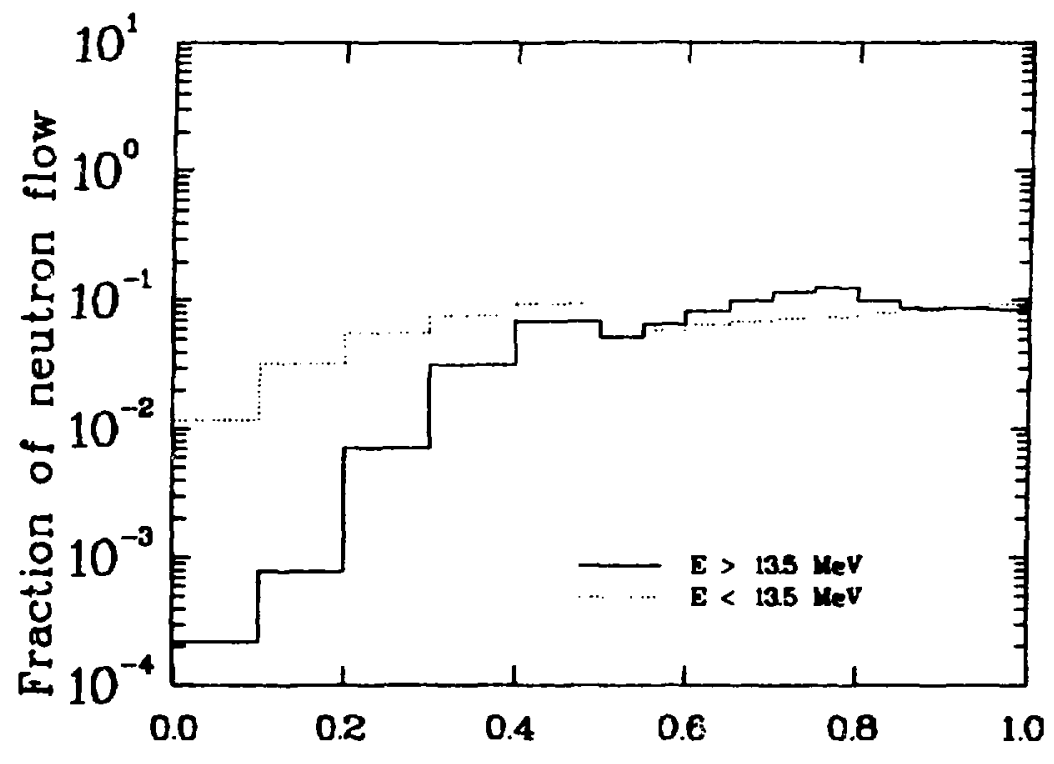

\section{Cosine(theta)}

Fig. 6. Angular distribution of neutrons at the outboard shield first wall. Theta is the angle between the normal to the trapping surface and the neutron direction. Each curve is normalized to one neutron. (MCNP results.) 


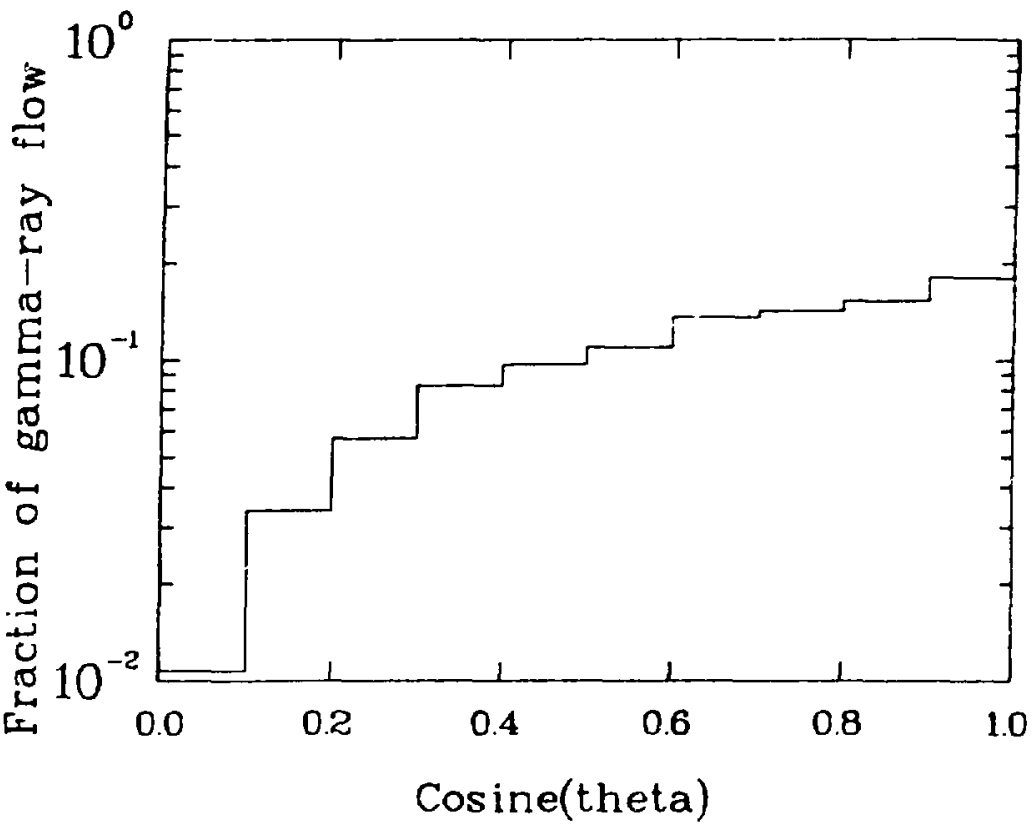

Fig. 7. Angular distribution of ganma rays crossing, in an outboard direction, the torus outboard shield first wall at and around the NBI duct mouth. Normalized to one gamma ray. (MCNP results)

Figs. 6 and 7 are the spatial averages over the trapping surface as upon close examination, no significant spatial variation of the angular distributions over the trapping surface was found.

Although it is the radiation flow that is important in determining the secondary source to be used in continuing the calculation, flux is a more commonly reported quantity. Figures 9 and 10 present, for information, the absolute neutron and gamma-ray flux spectrum at the trapping surface. The energy-integrated neutron and gamma-ray flux values are $1.55 \times 10^{15}$ neutrons $/ \mathrm{cm}^{2} \mathrm{~s}$ and $4.63 \times 10^{14}$ gamma rays $/ \mathrm{cm}^{2} \mathrm{~s}$, respectively. The fraction of the neutron flux in the energy range 13.5 to $15.0 \mathrm{MeV}$ is approximately 0.11 and all but about $1 \%$ of Lhese neutrons are at an energy of $14 \mathrm{MeV}$; i.e., the plasma source neutron starting energy. The total neutron and gamma-ray fluxes are approximately a factor of four times larger than the corresponding neutron and gamma-ray flows. Recall 


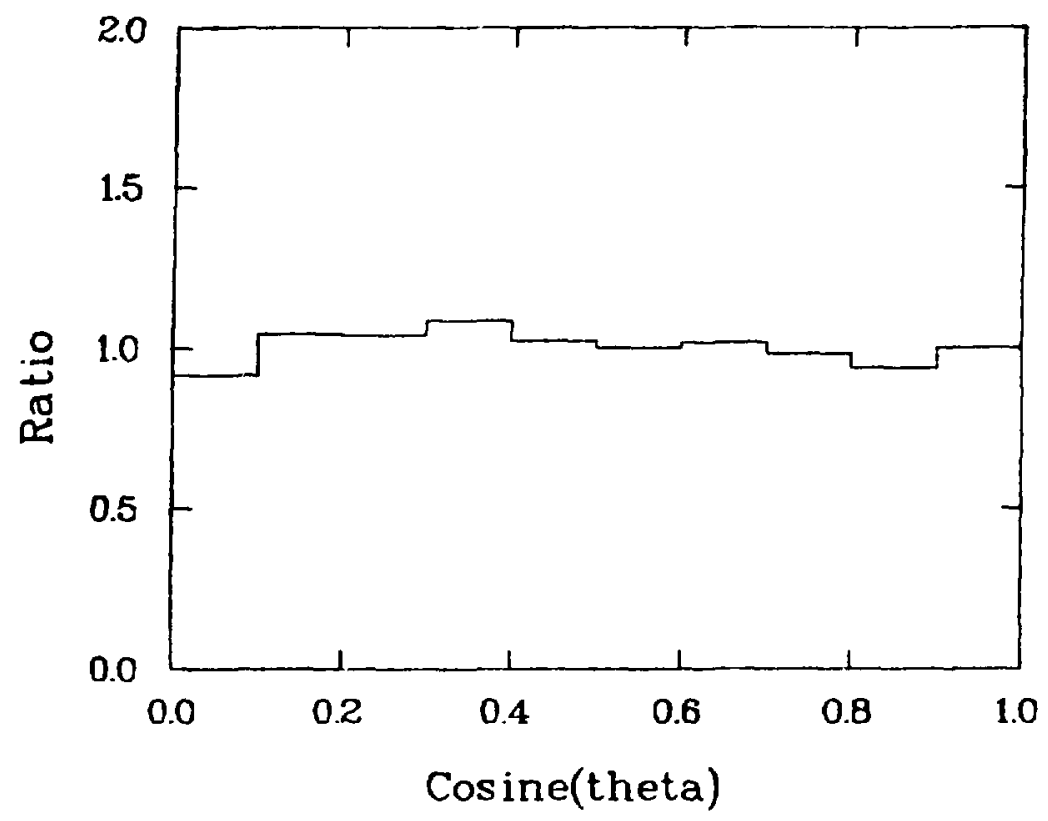

Fig. 8. Ratio of gamma ray to neutron ( $<<13.5 \mathrm{MeV}$ ) angular distributions at the torus outboard shield first wall. (MCNP results.)

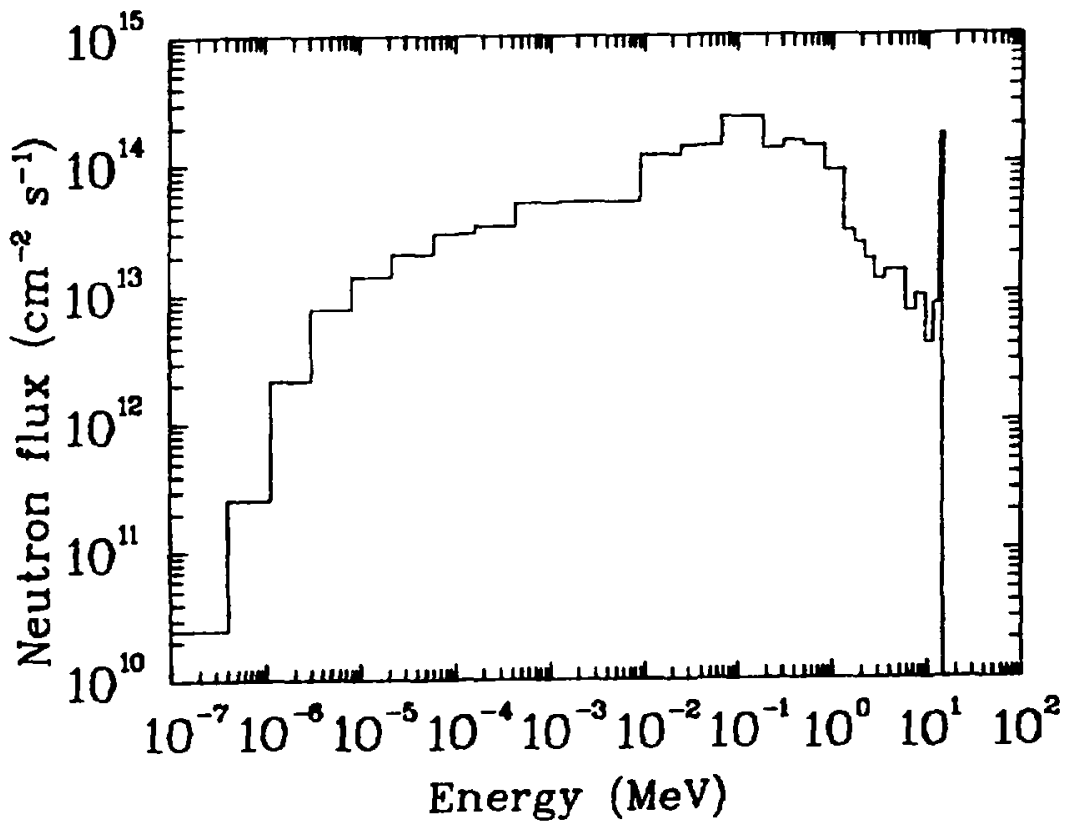

Fig. 9. Neutron flux spectrum at the torus outboard shield first wall, at and around the NBI-duct mouth. (MCNP results.) 


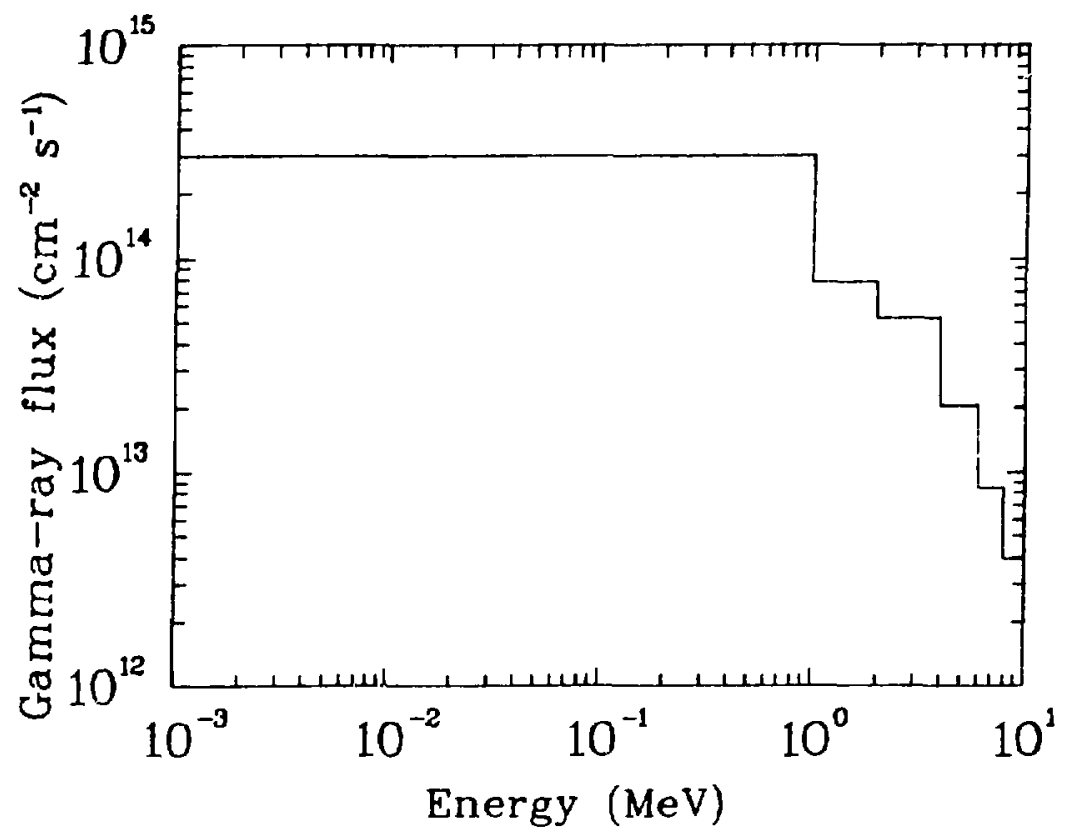

Fig. 10. Gamma-ray flux spectrum at the torus outboard shield first wall, at and around the NBI-duct mouth. (MCNP results.)

that the flow only accounts for particles going in the outboard direction, whereas the flux considers particles going in both the inboard and outboard directions across the surface of interest and weights each particle by the secant of its angle with respect to the surface normal.

\section{B. NBI-Duct Wall Surface Source Calculations}

The NBI-duct wall surface source for use with TRIDENT-CTR was generated from radiation flow incident on the NBI-duct walls. The geometry used to calculate the incident angular distribution consists of a rectangular duct $(0.8$ by $1.2 \mathrm{~m}$ by $6.65 \mathrm{~m}$ in length) with the source plane at one end. This geometry is illustrated in Fig. 11. It is important to note that the surface source is not perpendicular to the duct centerline and that it is really a cylindrical surface segment resulting from the duct intersection with the torus outboard shield inner radius (see also Fig. 3 ). This NBI model contains neither the duct shield nor the torus outboard shield, so once particles crossed the duct wall, their histories were terminated. 


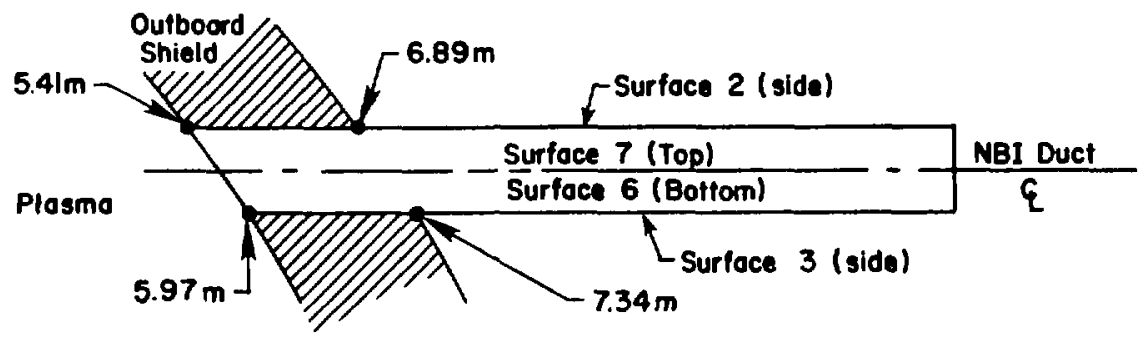

Plan View

Fig. 11. Schematic of NBI duct illustrating surface numbers of the duct walls and extent of outboard shield (not to scale).

Although the calculation is simply one of ray tracing, a considerable number of edits are required to obtain the spatially dependent angular distribution of particles crossing the walls of the duct. Also, variance reduction techniques such as splitting are not easily applicable in this problem. Hence, a large number of source particles are required to obtain reasonable statistics in the spatially dependent angular bins.

To achieve statistically reasonable results using the trapped particles from the torus trapping surface would require long machine runs with the torus geometry. However, the energy and angular distribution of neutrons and gamma rays entering the NBI duct were known as a result of the torus calculation described previously. Therefore, what was done was to construct semi analytic angular distributions based on the torus trapping surface results and to use these angular distributions for the NBI calculations. The distributions were assumed to be azimuthally symmetric with respect to the normal to the trapping surface source. Furthermore, the source was assumed to be spatially uniform, which is consistent with the results of the torus calculation. 
Two MCNP calculations were then performed using these semianalytic sources. Note that the calculations were energy independent as all that is needed from the calculation is the spatially dependent angular distribution of particles incident on the walls of the duct. Also recall that the angular distribution for neutrons with energies less than $13.5 \mathrm{MeV}$ is applicable to the gamma rays as discussed previously. In each case 250000 particles were started. As the particles crossed the duct walls they were tallied according to the wall crossed, spatial segment, and angular bin. Fifteen angular bins were considered and were chosen to represent an $S_{10}$ quadrature for TRIDENT-CTR.

Following each calculation, the data for the four walls were averaged to obtain a single spatially dependent (along the NBI-duct axis) angular distribution. This distribution, together with the appropriate neutron and gamma-ray energy distributions from the torus run, provided the basis for the NBI-duct wall surface source for TRIDENT-CTR.

There follows a presentation of a number of figures depicting the incident flow of particles on the duct walls. The surface numbers referred to in these figures are defined in Fig. 11. Furthermore, it should be noted that those histograms labeled "less than $13.5 \mathrm{MeV}^{\prime}$ are also representative of the gamma-ray distribution. The ordinate in all cases is relative with the exception of the ratio plots or where otherwise noted. When referring to these histograms, the extent of the outboard shield along the duct can be found by referring back to Fig. 11. Finally, the fractional errors for the spatial segment data plotted in Figs. 12 through 16 vary from less than $0.5 \%$ near the source to $7 \%$ for the segment furthest from the source.

Although not of direct use in the surface source generation for TRIDENTCTR, it is of interest to examine the incident spatial distribution of particles on each individual duct wall. Figures 12 and 13 contain the relative flow of particles incident on each of the four walls as a function of distance along the duct axis for the greater than, and less than, 13.5-MeV neutron source angular distributions, respectively. Figure 14 presents the four-wall average spatial distributions for these two sources.

Because the TRIDENT - CTR NBI duct calculation is a 2 - D calculation, the inner duct wall is represented as a cylinder whose axis is congruent with the rectangular NBI-duct axis. Consequently, there is azimuthal symmetry 


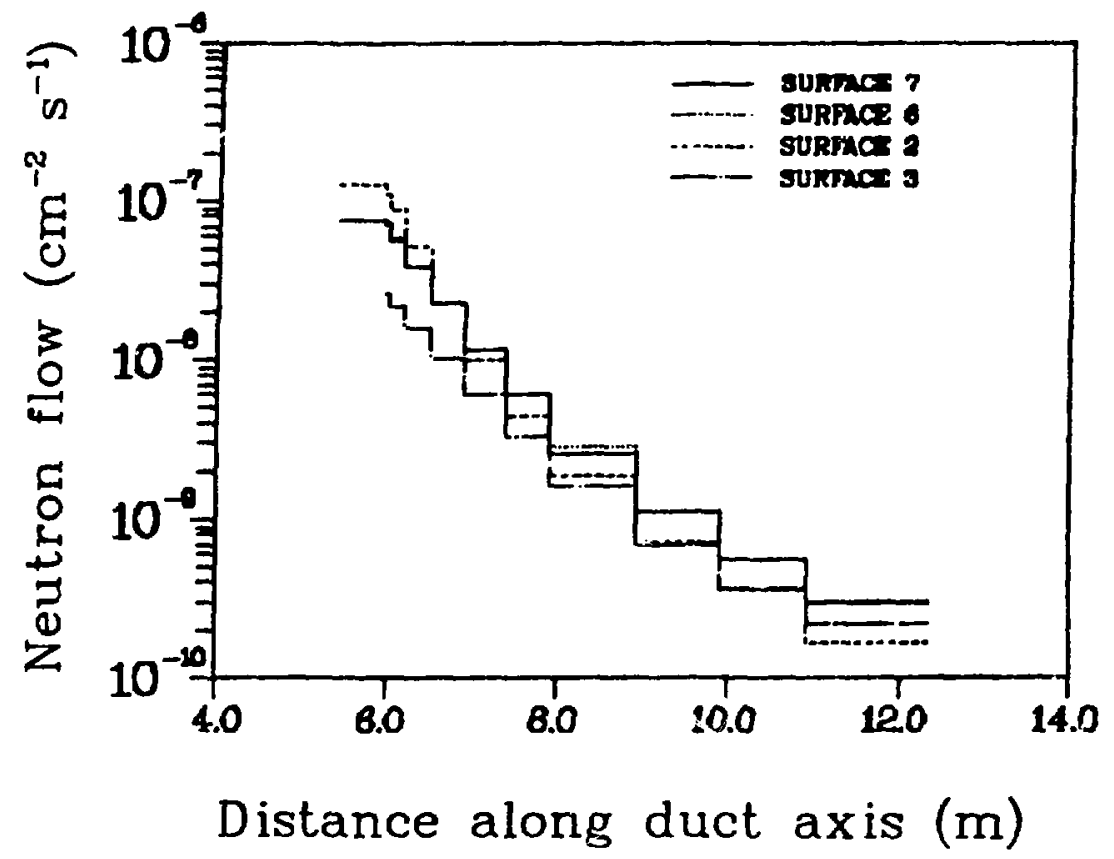

Fig. 12. Neutron flow incident on the NBI duct walls as determined by MCNP using the semianalytic source angular distribution for neutron energies greater than $13.5 \mathrm{MeV}$.

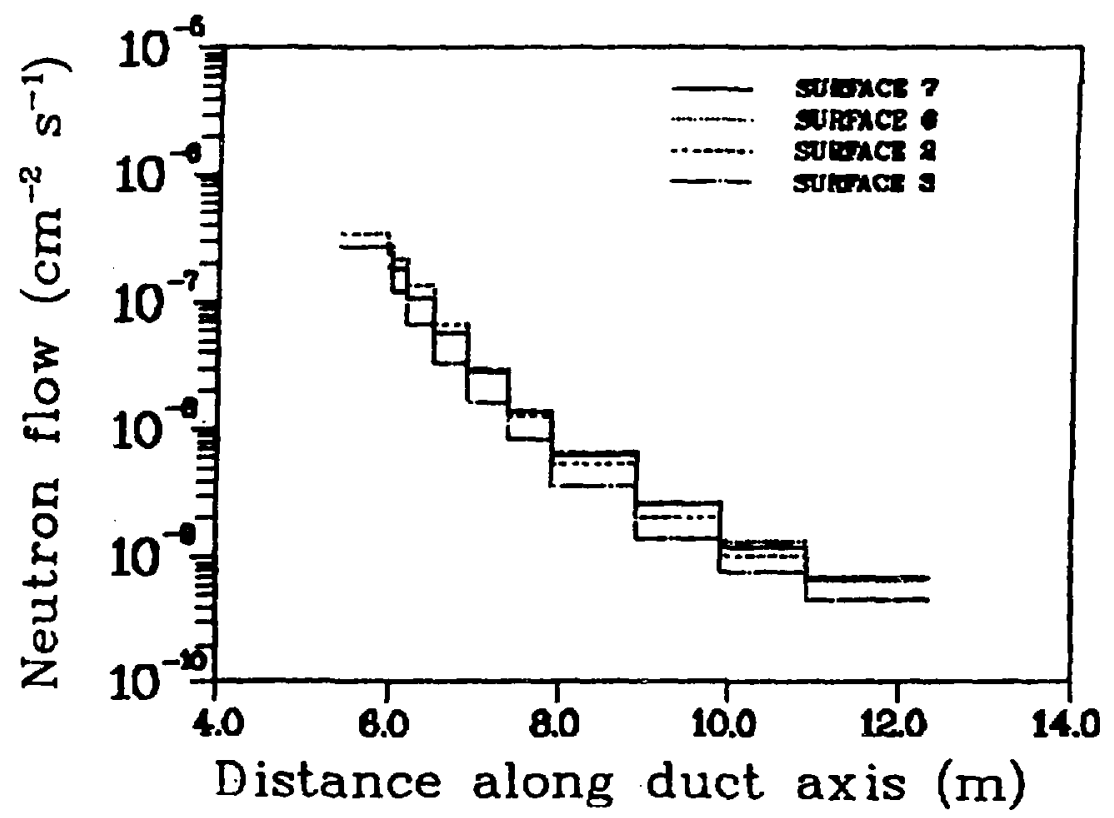

Fig. 13. Neutron flow incident on the NBI duct walls as determined by MCNP using the semi-analytic source angular distribution for neutron energies less than $13.5 \mathrm{MeV}$ (and for gamma rays). 


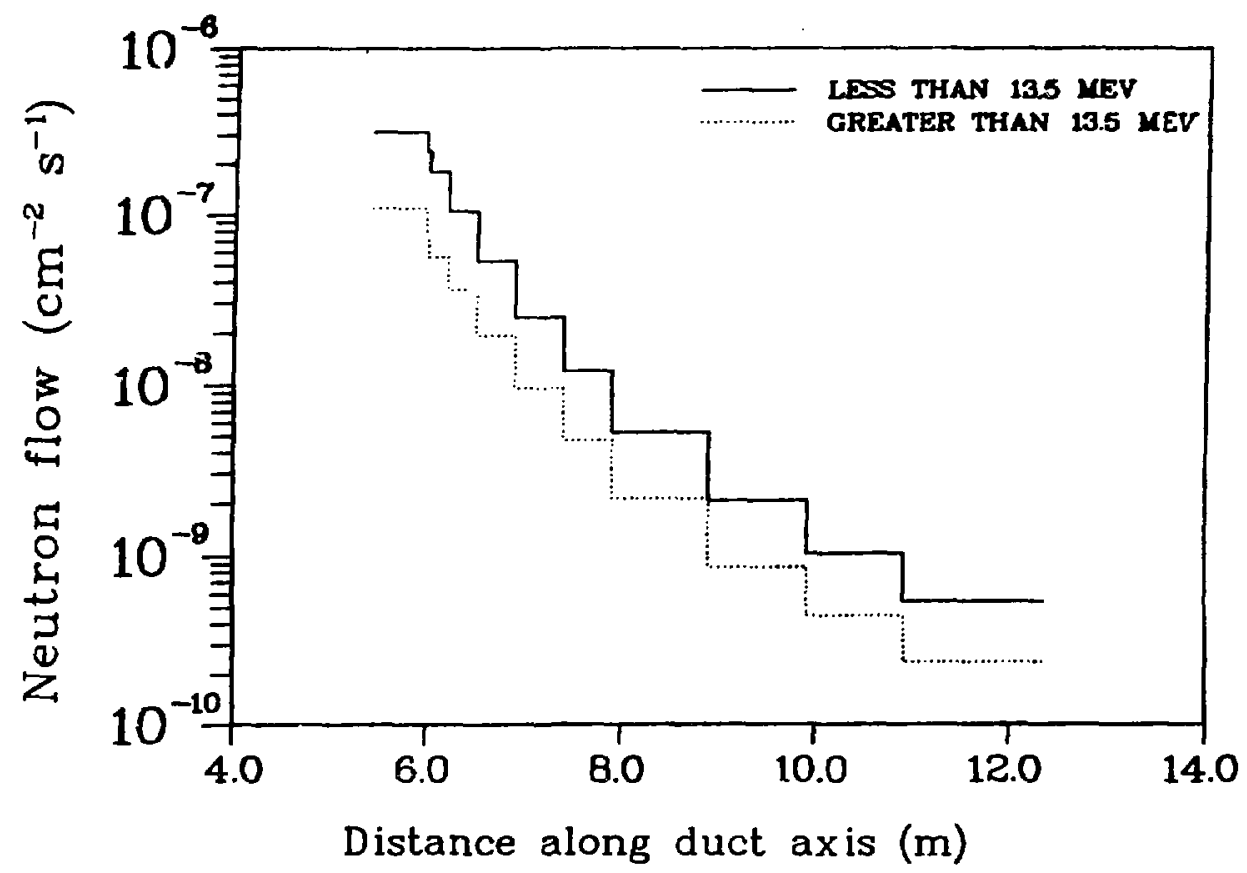

Fig. 14. Average neutron flow incident on the four NBI-duct walls as determined by MCNP using the semianalytic source angular distributions for neutrons less than, and greater than, $13.5 \mathrm{MeV}$.

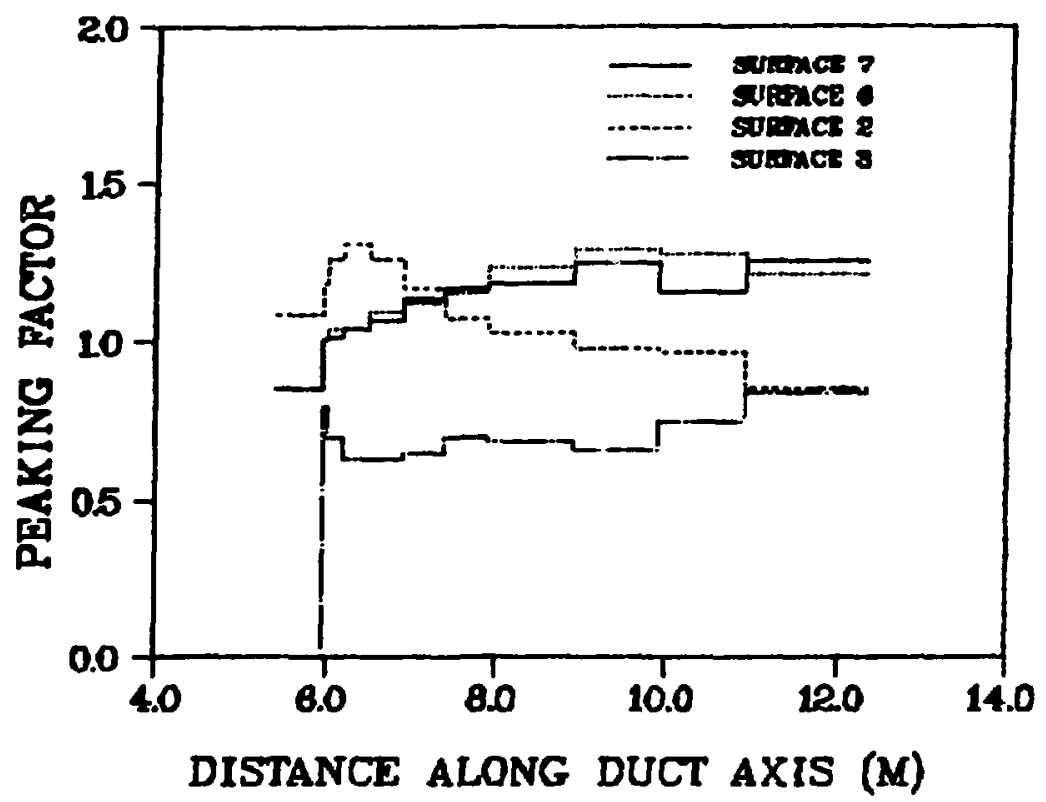

Fig. 15. Peaking factor versus distance along the NBI duct as determined from the MCNP calculations using the semianalytic source angular distribution for neutrons less than $13.5 \mathrm{MeV}$ (and for gamma rays). 


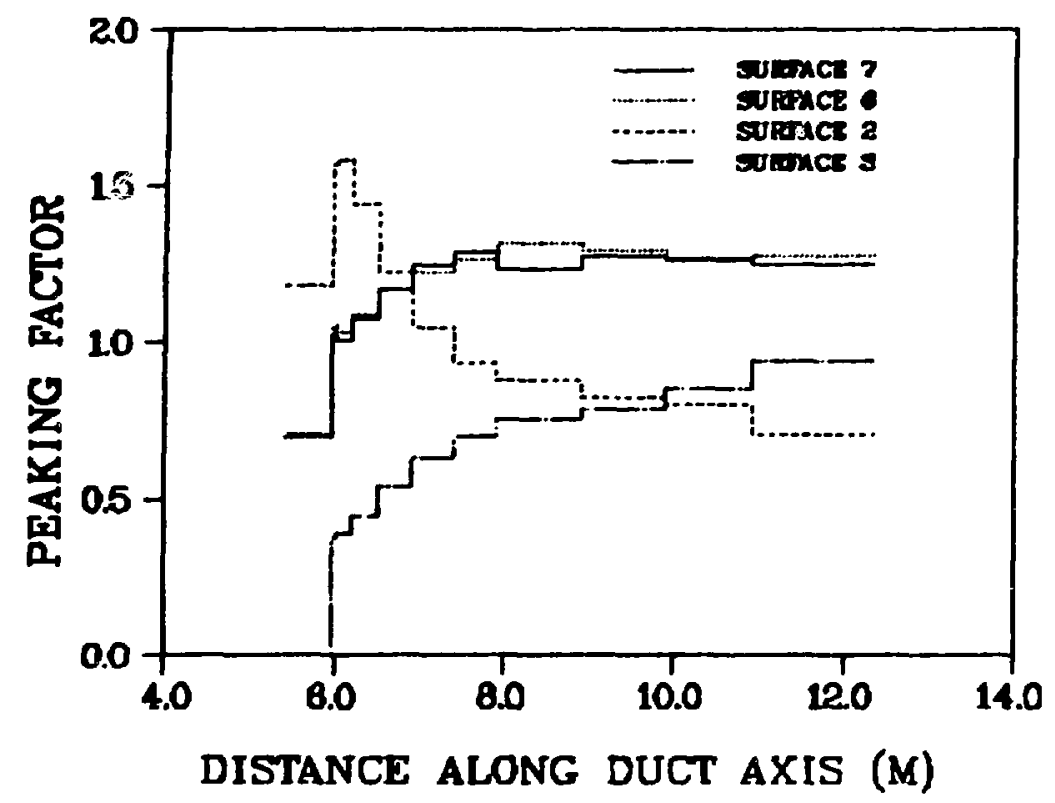

Fig. 16. Peaking factor versus distance along the NBI duct as determined from the MCNP calculations using the semianalytic source angular distribution for neutron energies greater than $13.5 \mathrm{MeV}$.

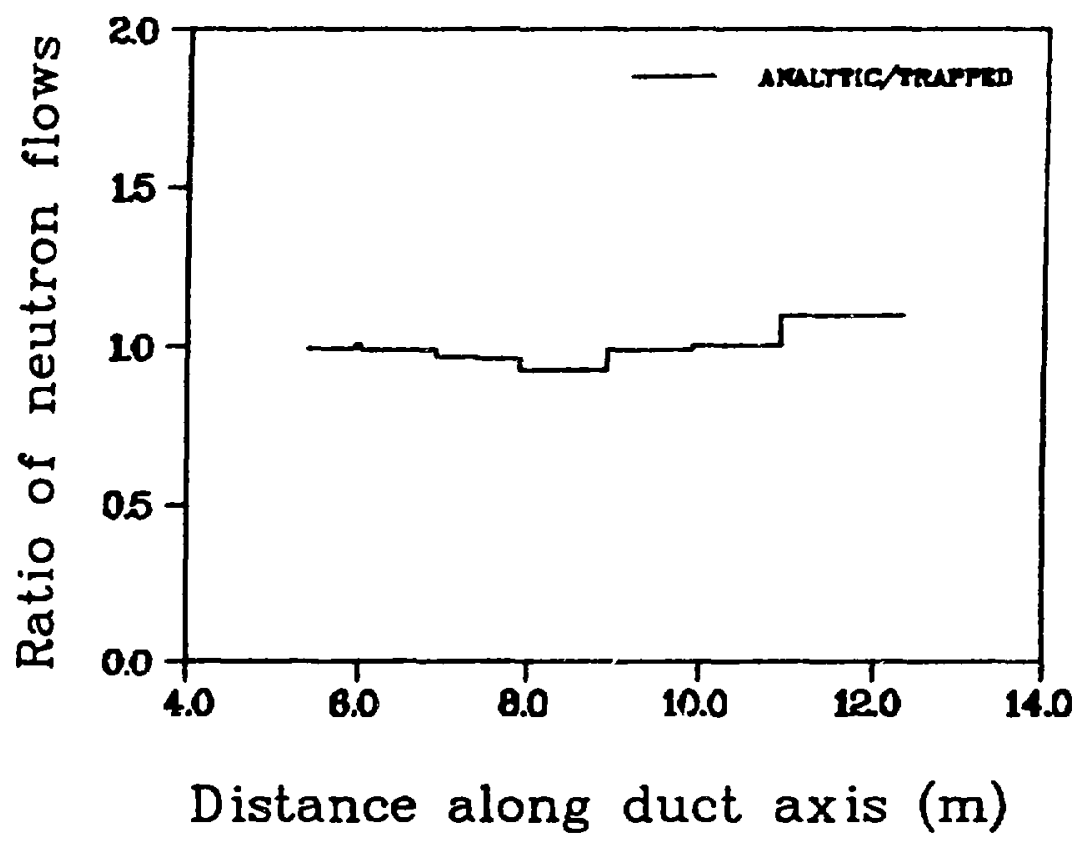

Fig. 17. Semianalytic source angular distribution to trapped source four-wall average neutron flow versus distance along the duct axis for neutron energies less than $13.5 \mathrm{MeV}$. 
about the $z$-axis both in geometry and in the source. Therefore, it is of interest to the shield designer to know the variation of the incident neutron magnitude on each of the four duct walls relative to the average value used in determining the surface source. This information is provided in Figs. 15 and 16 wherein the peaking factor (i.e., the ratio of the incident number of particles $/ \mathrm{cm}^{2}$ for each wall to the average number of particles $/ \mathrm{cm}^{2}$ is plotted versus distance along the duct axis for the two source angular distributions. These two figures again illustrate that the relative number of particles incident on each of the duct walls is different and that their relationships to one another also change with distance from the duct routh. When examining these figures one should keep in mind the orientation of the source plane relative to the duct axis and the extent of the outboard shield as previously discussed; i.e., refer to Fig. 11 .

In the calculations just described, the source angular distribution in each case was assumed to be azimuthally symmetric about the normal to the source plane. This assumption is, of course, an approximation to reality, but the effect of this approximation is shown below to be minimal. This is because the angular distributions for the four duct walls are averaged tr obtain the spatially dependent angular distribution for use in determining the surface source for TRIDENT-CTR. An estimate of the error introduced by this approximation can be obtained by comparing the four-wall average spatial distributions of Fig. 14 with similar four-wall average data obtained using the actual trapped neutrons from the torus calculation. Figure 17 presents a ratio of these quantities, the semianalytic and trapped source calculated results, for the case of neutron energies less than $13.5 \mathrm{MeV}$. The agreement is quite gooc, with the ratio having a value within $\sim 6 \%$ of 1.0 for all spatial segments but the one furthest from the duct mouth (i.e., the source plane), where the ratio is 1.12. Figure 18 provides the same ratio for neutrons above $13.5 \mathrm{MeV}$. Except for the last 4 segments the ratio is within $\sim 12 \%$ of 1.0 . In the last 4 segments, i.e., beyond $7.9 \mathrm{~m}$, the four-wall average data obtained using the trapped neutrons as a source have fractional errors greater than $10 \%$ and get progressively larger, thereby making the ratio for this region of the histogram statistically less reliable.

Peaking factors were also computed for each of the four walls using the trapped neutron source. As would be expected from the foregoing discussion, beyond several meters down the duct the peaking factor data become statistically unreliable and all that can be concluded is that the surface-by-surface peaking 


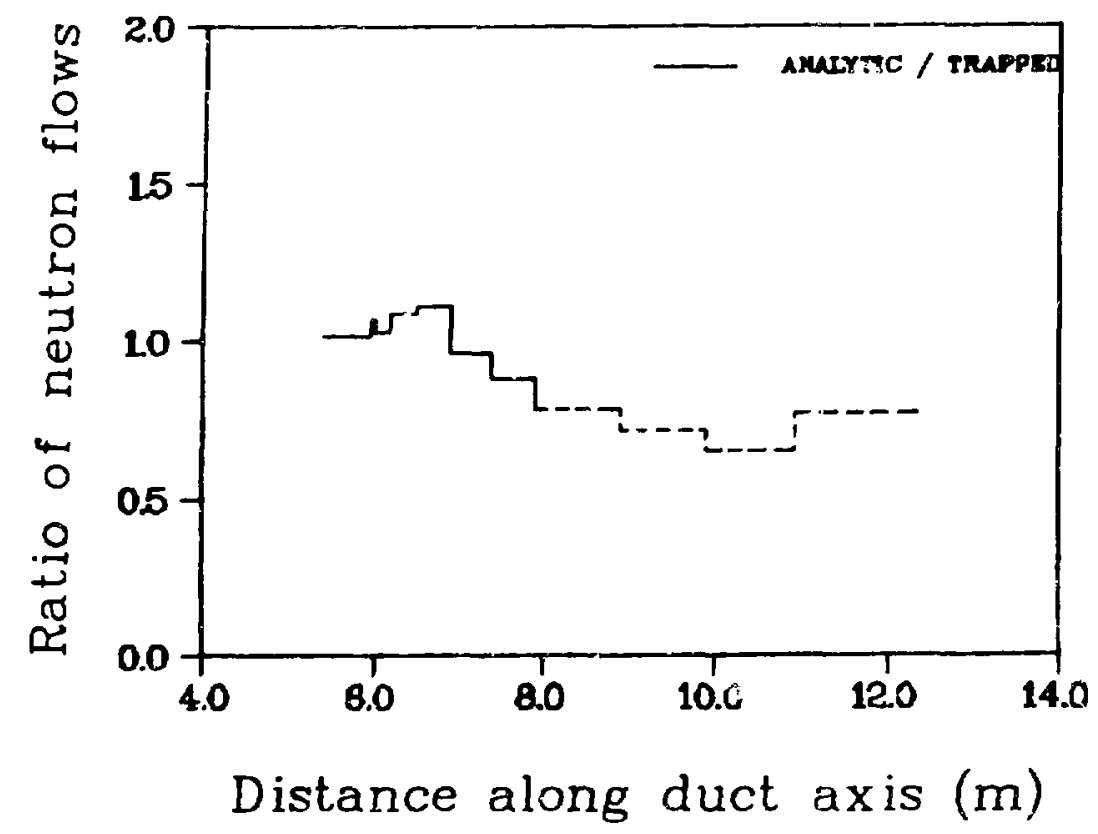

Fig. 18. Semianalytic source angular distribution to trapped source four-wall average neutron flow versus distance along the duct axis for neutron energies greater than $13.5 \mathrm{MeV}$ (values at distances greater tha.i $7.9 \mathrm{~m}$ statistically less reliable).

factors exhibit very similar behavior at distances close to the source as that observed for the peaking factors derived from the calculations that used the semianalytic source.

\section{NBI-Duct Shield Calculations}

The purpose of the MCNP NBI-duct shield calculation was to allow an intercomparison of the MCNP results with the TRIDENT-CTR results in the duct shield, and to obtain data from : shich a source could be constructed for use in the VPduct calculations. Hence, in this calculation the transport of radiation in the shield was allowed. To obtain the data required for continuing the MCNP calculations into the VP duct, a trapping surface was located at and around the NBI-duct/VP-duct interface. 
The MCNP model for this calculation is shown in Fig. 19. The 0.5-m-thick shield consists of $0.125 \mathrm{~m}$ of stainless steel followed by $0.375 \mathrm{~m}$ of a homogeneous mixture of stainless steel and borated water. The 0.8 -m-thick shield consists of $0.2 \mathrm{~m}$ of stainless steel and $0.6 \mathrm{~m}$ of a homogeneous mixture of stainless steel and borated water (see Table II). The stainless steel adjacent to the plasma is $0.3-\mathrm{m}$ thick. This andel approximates the conceptual NBI shield wherein the material thicknesses of the model are the arithmetic average of the corresponding tapers. The effect of this approximation is expected to be minimized because (1) over most of the duct length all that is needed is the albedo from the shield and (2) at those locations in the shield where MCNP/ TRIDENT-CTR comparisons are to be made, the two shield thicknesses are almost identical.
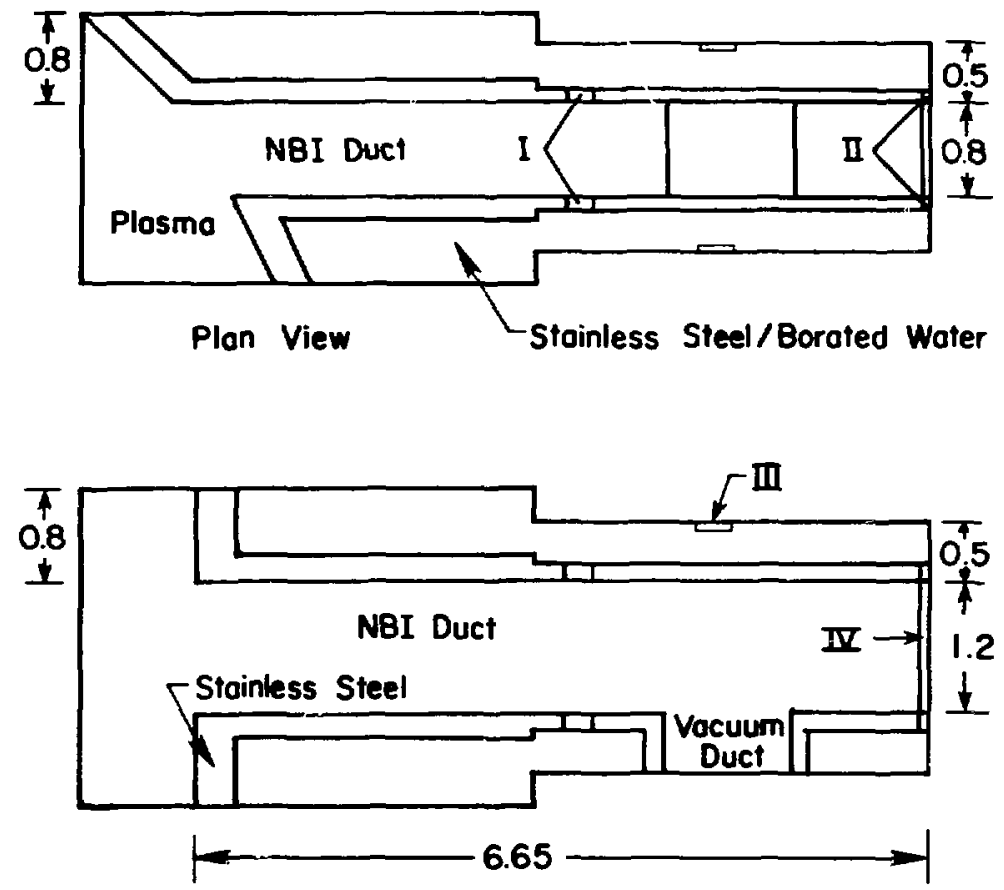

Elevation View

Fig. 19. Plan and elevation views of the NBI duct and shielding MCNP model. The Roman numerals identify those regions used for MCNP/TRIDENT-CTR comparisons. (Dimensions in metres.) 
Two different calculations were made using this model; one was a coupled neutron/gamma-ray calculation and the other was a gama-ray calculation. Two calculations were necessary because MCNP does not readily allow a source consisting of both neutrons and gamma rays. For both calculations, a secondary source plane at and around the torus outboard shield first-wall/NBI-duct interface was used. The source consisted of either the trapped neutrons or the trapped ganma rays from the torus MCNP calculations. Splitting and Russian Roulette coupled with particle cut-offs constituted the primary variance reductiun technique used in the calculations.

Neutron and gamma-ray fluxes were edited at selected locations for comparision with. TRIDENT-CTR. Total neutron and gamma-ray heating values were also obtained. These results were normalized to represent an incident neutron flow rate on the torus outboard shield first wall due to neutrons with entrgies between 13.5 and $15.0 \mathrm{MeV}$ of $\sim 2.4 \mathrm{MW} / \mathrm{m}^{2}$. Neutrons and gamma rays crossing the trapping surface at and around the NBI-duct/VP-duct interface were saved; i.e., their spatial coordinates, direction cosines, energy, weight, and time were written to a file. These data then provide the basis for a secondary source plane at the VP-duct mouth.

\section{VP-Duct Wall Surface Source Calculations}

The VP-duct wall surface source for use with TRIDENT-CTR was generated from the results of MCNP calculations, which determined the angular distribution of radiation flow incident on the VP-duct walls. These calculations are very similar to those described in Section III.B for the NBI duct.

The geometric model for this calculation consists of a rectangular duct $(0.8$ by $1.2 \mathrm{~m}$ by $3.3 \mathrm{~m}$ in length) with the source plane at the end. This model is illustrated in Fig. 20. The model does not contain the VP-duct shield, so once particles crossed the duct wall, their histories were terminated.

The neutron and gamma-ray sources used consisted of the trapped particles entering the vacuum duct at the NBI-duct/VP-duct interface as obtained from the NBI-duct calculations described in Section III.C. This is a departure from the procedure used in the NBI calculations of Section III.B wherein semianalytic source angular distributions, based on the source angular distributions of the trapped particles, were used. The appizch taken for this VP-duct calculation was judged to be adequate based on the lessons learned 


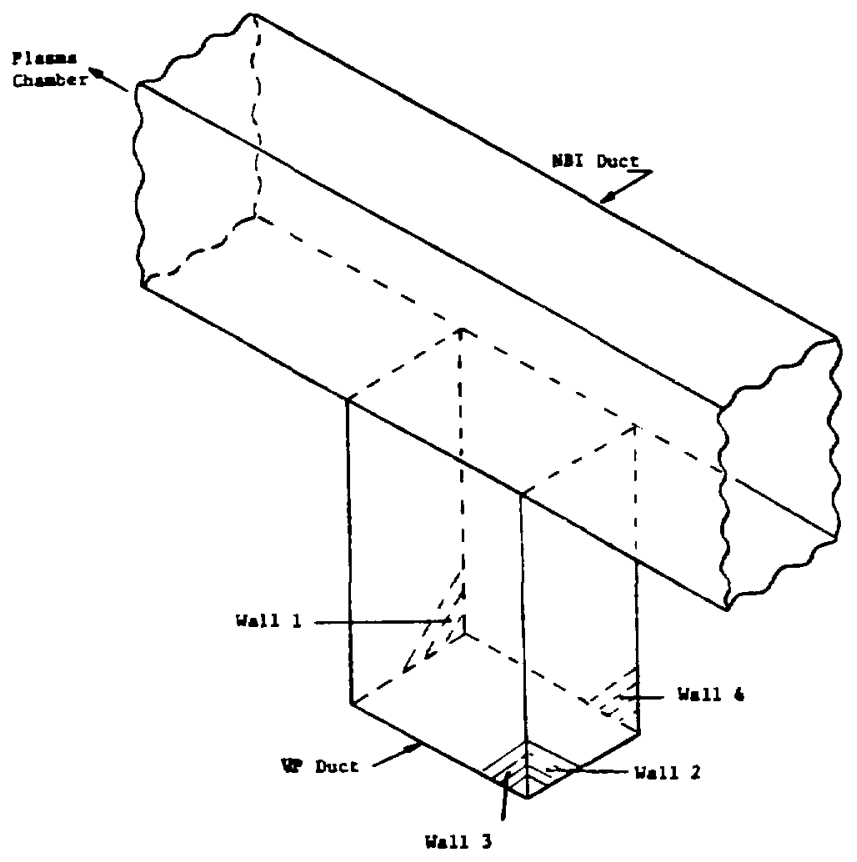

Fig. 20. VP-duct MCNP model used to obtain TRIDENT-CTR sources. NBI duct not included in the model but shown to provide a frame of reference.

from the NBI calculations and because the neutrons entering the VP duct had a much softer spectrum and the duct was much shorter in length. It was assumed that the angular distribution of gamma rays incident on the duct walls was the same as that for the neutrons with energies less than $13.5 \mathrm{MeV}$. This assumption was also made for the NBI-duct calculations (see Section III. B) based upon a comparison of the neutron and garma-ray source angular distribution data. The neutron calculation tallied the neutrons as they crossed the duct wall according to the wall crossed, spatial segment, energy bin and angular bin. One energy bin above $13.5 \mathrm{MeV}$ and one below $13.5 \mathrm{MeV}$ were considered. Fifteen angular bins were considered and were chosen to represent an $s_{10}$ quadrature for TRIDENT-CTR .

Energy, angular and spatially dependent distributions of neutrons incident on each of the four duct walls were thus provided for use in generating the required surface sources for TRIDENT-CTR. However, it was decided to take a conservative approach with TRIDENT-CTR and make the calculation only for the "hot" wall (i.e., surface 2 in Fig. 20) as discussed in Section IV.B. 
The results of this intermediate MCNP calculation are presented in Figs. 21 through 24. The relative flow of neutrons incident on surface 2 for two energy groups (i.e., above $13.5 \mathrm{MeV}$ and below $15.0 \mathrm{MeV}$ ) is shown as a function of distance from the duct mouth in Fig. 21. Tt energy distribution of these neutrons averaged over the entire surface of wall 2 is shown in Fig. 22 . This spectrum is normalized to 1.0 incident neutron. These two figures clearly show that only $\approx 13 \%$ of the total neutrons incident on wall 2 have energies above 13.5 MeV and that this incidence of high-energy neutrons is restricted to the first $0.6 \mathrm{~m}$ of this wall (relative to the VP-duct mouth). Furthermore, only $\sim 3 \%$ of the neutrons incident on this wall have energies between 1.353 and $13.5 \mathrm{MeV}$, $79 \%$ have energies between $6.14 \times 10^{-5}$ and $1.353 \mathrm{MeV}$ and the remaining $5 \%$ are below $61.4 \mathrm{eV}$. Because neutron penetration through a shield is so strongly energy dependent, the information in these two figures quickly provides a clue as to the area on the outside of the VP-duct shield that will have the highest radiation levels. This is discussed further in section IV.B. The spatial data in Fig. 21 all have errors less than $10 \%$ except for the next

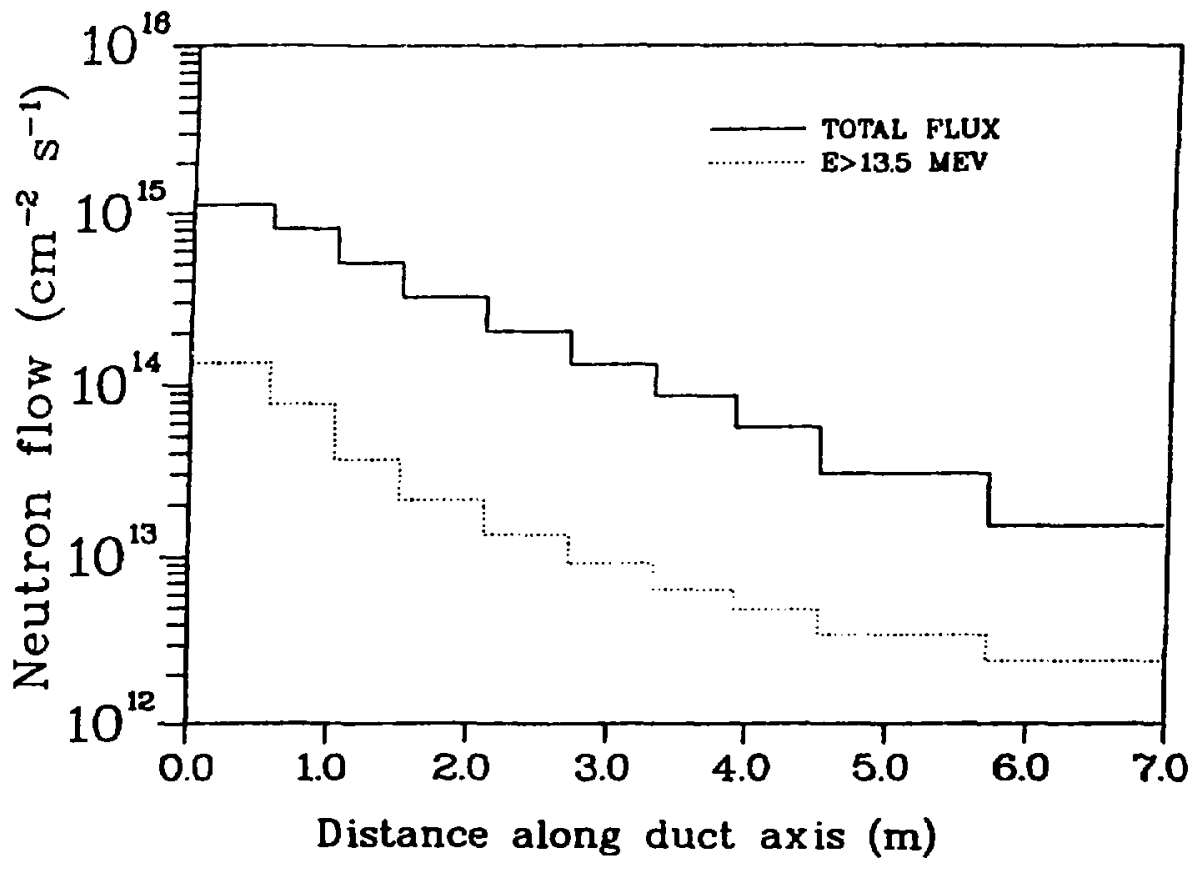

Fig. 21. Neutron flow incident on vacuum duct wall 2 versus distance from the duct mouth; i.e., the NBI-duct/vacuum-duct interface. See Fig. 20 for wall indentification. (MCNP results.) 


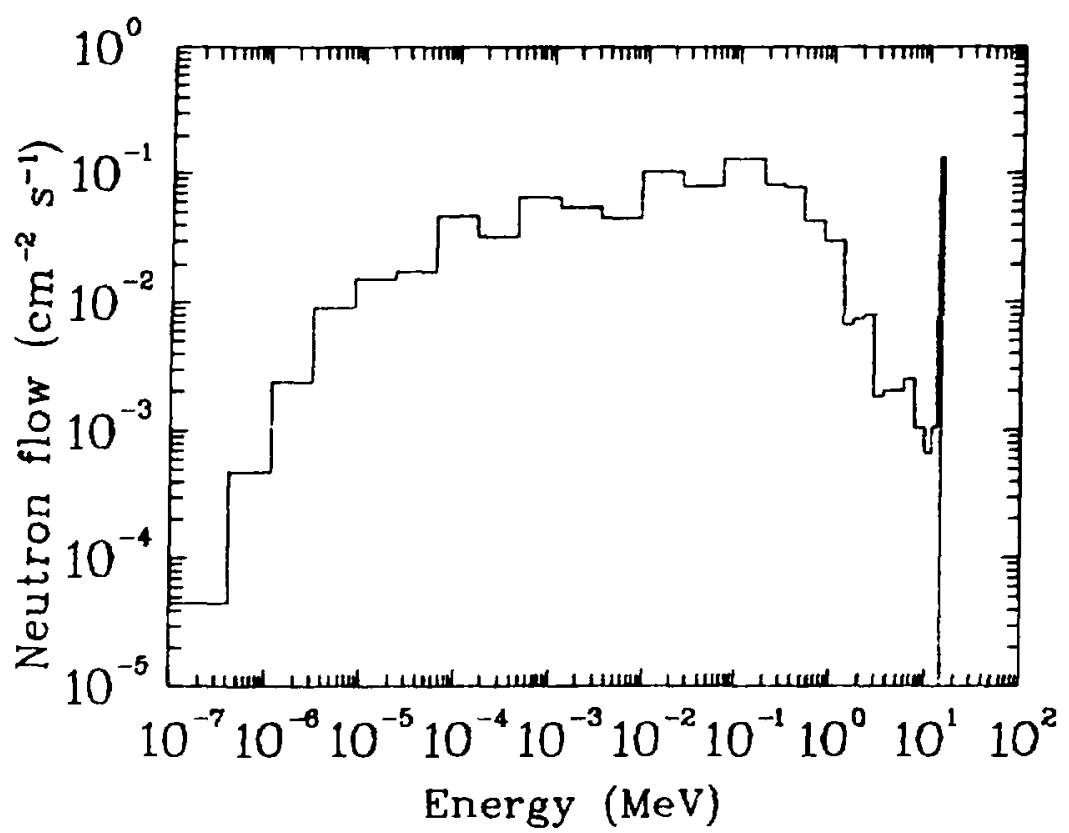

Fig. 22. Energy spectrum of the initial incident flow of neutrons from the NBI duct on VP-duct wall 2. Normaiized to 1.0 incident neutron. See Fig. 20 for VP duct wall indentification. (MCNP results.)

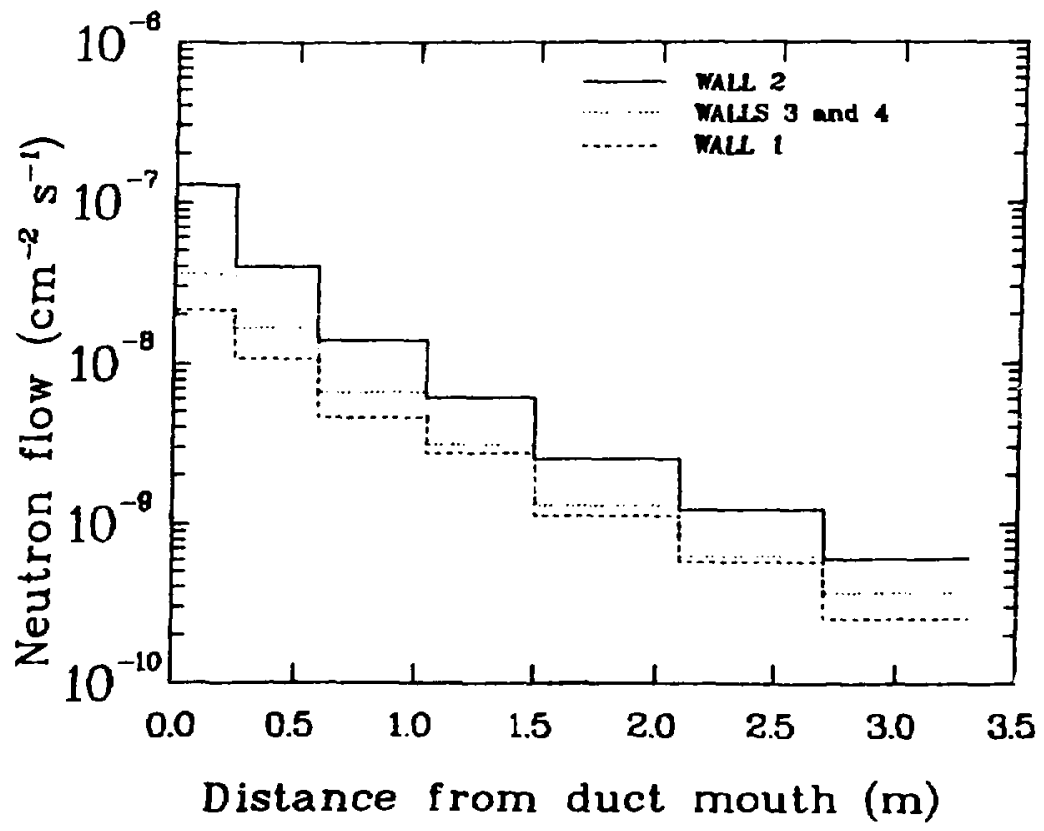

Fig. 23. Comparison of the relative incident flow of neutrons from the NBI duct on the VP-duct walls. See Fig. 20 for duct wall identification. (MCNP results.) 


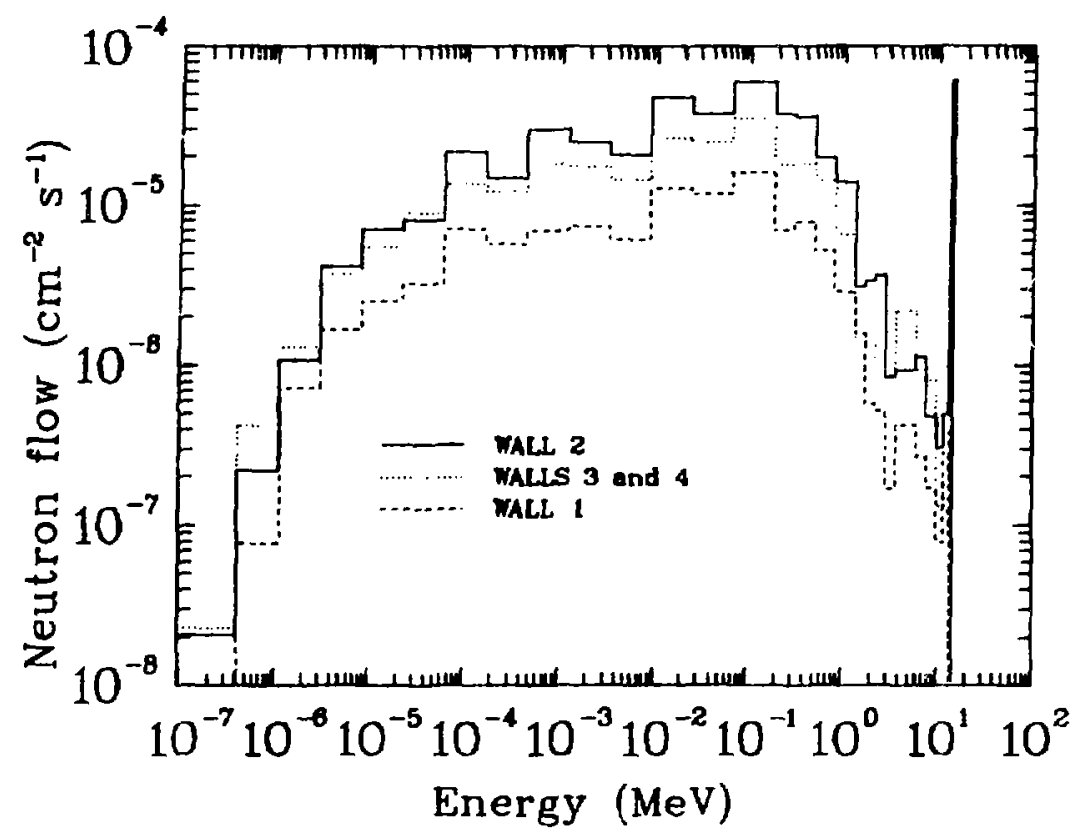

Fig. 24. Comparison of the relative energy spectra of initial incident flow of NBI neutrons on the VP-duct walls. See Fig. 20 for VP duct wall identification. (MCNP results.)

to the last segment $(11 \%)$ and the last segment $(15 \%)$. With the exception of the energy bins below $1.0 \mathrm{eV}$ and between 1.353 and $13.5 \mathrm{MeV}$, the spectrum groupwise data have errors of less than $8 \%$ and the error associated with the total spectrum is $1 \%$.

The spatially dependent neutron flows incident on each of the VP-duct walls are compared in Fig. 23, whereas the energy spectra over each of the walls are compared in Fig. 24. Because the data for walls 3 and 4 are statistically indistinguishable, their data are represented by a single curve, which is the average of the wall 3 and 4 data. These figures illustrate graphically that not only are more neutrons incident on wall 2 but also that they have a harder spectrum as compared to the other three walls. The fractions of the total number of neutrons that enter the duct and are initially incident on walls $1,2,3$, and 4 are $0.101,0.424,0.232$, and 0.226 , respective1y. The remaining fraction, i.e. 0.017 , escape from the duct into the VP chamber without 
crossing any of the duct walls. It is the information in Figs. 23 and 24 that led to the choice of wall 2 for the TRIDENT-CTR calculation. (See Section V.B.) The errors associated with the wall 1 and the wall 3-4 average are similar to those previously given for wall 2.

\section{E. VP-Duci Shield Calculations}

The VP-duct shield calculations differed from those described in Section III.D primarily in that neutron and gamma-ray transport in the shield was allowed. Also, the edits of this calculation were designed to provide a means of comparison with the TRIDENT-CTR VP-duct calculation.

The MCNP model for this calculation is shown in Fig. 25. As an aid to relating this model to the NBI duct and the torus, Figs. 19 and 20 are helpful. The 0.4-m-thick VP-duct shield consists of $0.1 \mathrm{~m}$ of stainless steel followed by $0.3 \mathrm{~m}$ of a homogeneous mixture of stainless steel and borated water. The stainless steel adjacent to the NBI duct is $0.125 \mathrm{~m}$ thick. Isotropic compositions of these materials are presented in Table II.

Two different calculations were made using this model; one was a coupled neutron/gamma-ray calculation and the other was a gamma-ray calculation. Two calculations were necessary for the reason explained in Section III.C. A secondary source plane at and around the NBI-duct/VP-duct interface was used in each calculation. The source consisted of either the neutrons or the gamma rays that resulted from the use of the NBI-duct/VP-duct interface trapping plane in the NBI-duct shield calculations described in Section III.C. Except for that portion of the neutron surface source that extends beyond the VP-duct cross-sectional area, the source is the same as that described in Section III.D. The extended source is used here to allow inclusion of the lip penetration contribution to the VP-duct results. Figures 21 through 24 provide an insight as to the spatial and energy dependence of neutrons incident on each of the four duct walls resulting from their first flight from the secondary source plane located at the duct mouth.

The MCNP calculation relied on splitting and Russian Roulette for variance reduction. Except at locations deep within the shield the number of trapped neutrons and gamma rays was sufficient to attain adequate statistics for the edit quantities of interest. The source file was used a second time, but the MCNP run was started with a different pseudo-random number, to reduce the relative errors of those regions deep within the shield. 


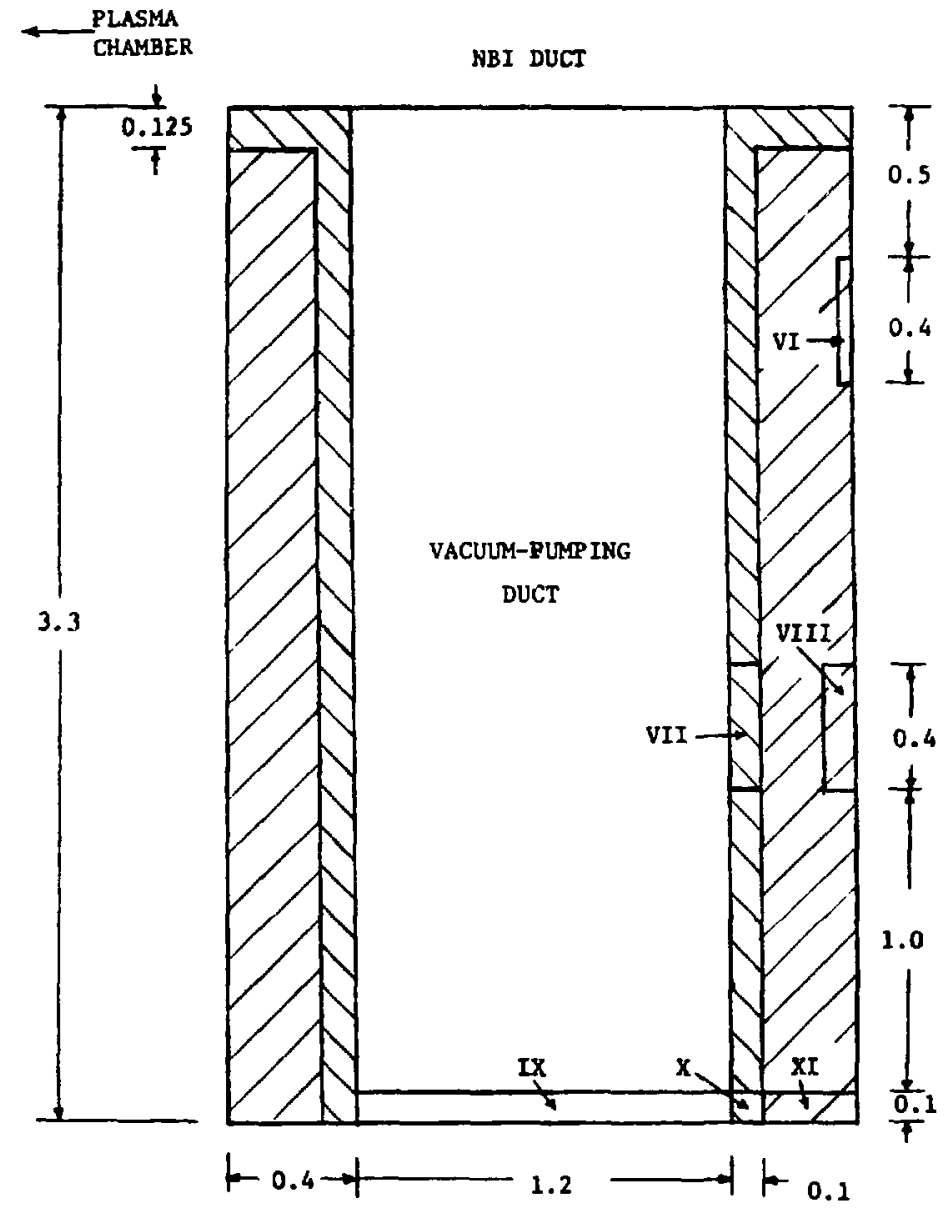

D Stainles, steel

7 stalnless steel/Borated Water

Fig. 25. Vacuum-pumping duct MCNP geometric model. Roman numerals identify those regions used for MCNP/TRIDENT-CTR comparisons. (Dimensions in metres.) 
Neutron and gamma-ray fluxes were edited along the VP duct and at those locations indicated by Roman numerals in Fig. 25. Total neutron and gamma-ray heating rates were also edited at those same locations in the shield. Results were normalized to represent an incident neutron flow rate on the torus outboard shield rirst wall because of neutrons with energies between 13.5 and $15.0 \mathrm{MeV}$ of of $\sim 2.4 \mathrm{MW} / \mathrm{m}^{2}$.

\section{TWO-DIMENSIONAL DISCRETE ORDINATES (TRIDENT-CTR) CALCULATIONS}

Two-dimensional calculations for both the NBI-duct shield and the VP-duct shield were performed to generate spatially detailed neutron and gamma-ray fluxes. These fluxes are required for the calculation of spatially dependent dose rates, heating rates, activation and other parameters of interest to the designers. TRIDENT-CTR is a two-dimensional, $(x, y)$ or $(r, z)$ geometry, multigroup neutral-particle transport code developed at Los Alamos for toroidal calculations. The use of triangular finite elements gives it the geometric flexibility to cope with the nonorthogonal shapes of many toroidal and nontoroidal designs of current interest in the Fusion Reactor community. This code was specifically used for the NBI-duct shield to allow accurate modeling of the shield tapers and the shutter shield; see Fig. 26. It was also required for both ducts because of its internal boundary source capabilities.

in order to pose the original problems in a form amenable to solution by TRIDENT-CTR [i.e., an $(r, z)$ geometry form], the rectangular cross section of both ducts was approximated by a circular cross section of the same area, the radius of which is $0.553 \mathrm{~m}$. The width of both duct shields was set to the design specifications. Both problems were modeled with the z-axis running down the center of the ducts and the $r$-axis traversing the duct shielding. The models used are shown in Figs. 26 and 27 , and the details particular to each duct problem are discussed in the following Sections; i.e., IV.A and IV.B.

The nuclear cross-section sets used for both the NBI-duct and VP-duct shield analyses were derived from the standard Los Alamos 30/12-group coupled neutron/gamma-ray MATXS 1ibrary, ${ }^{6}$ ising the TRANSX code ${ }^{7}$ on the NMFECC computers . $\mathrm{A}_{3}$ scattering order was used, with the cross sections transport corrected using the Bell, Hansen, and Sandmeier methodology. ${ }^{8}$ The energy group structure of the cross-section set is given in Table $I$. 

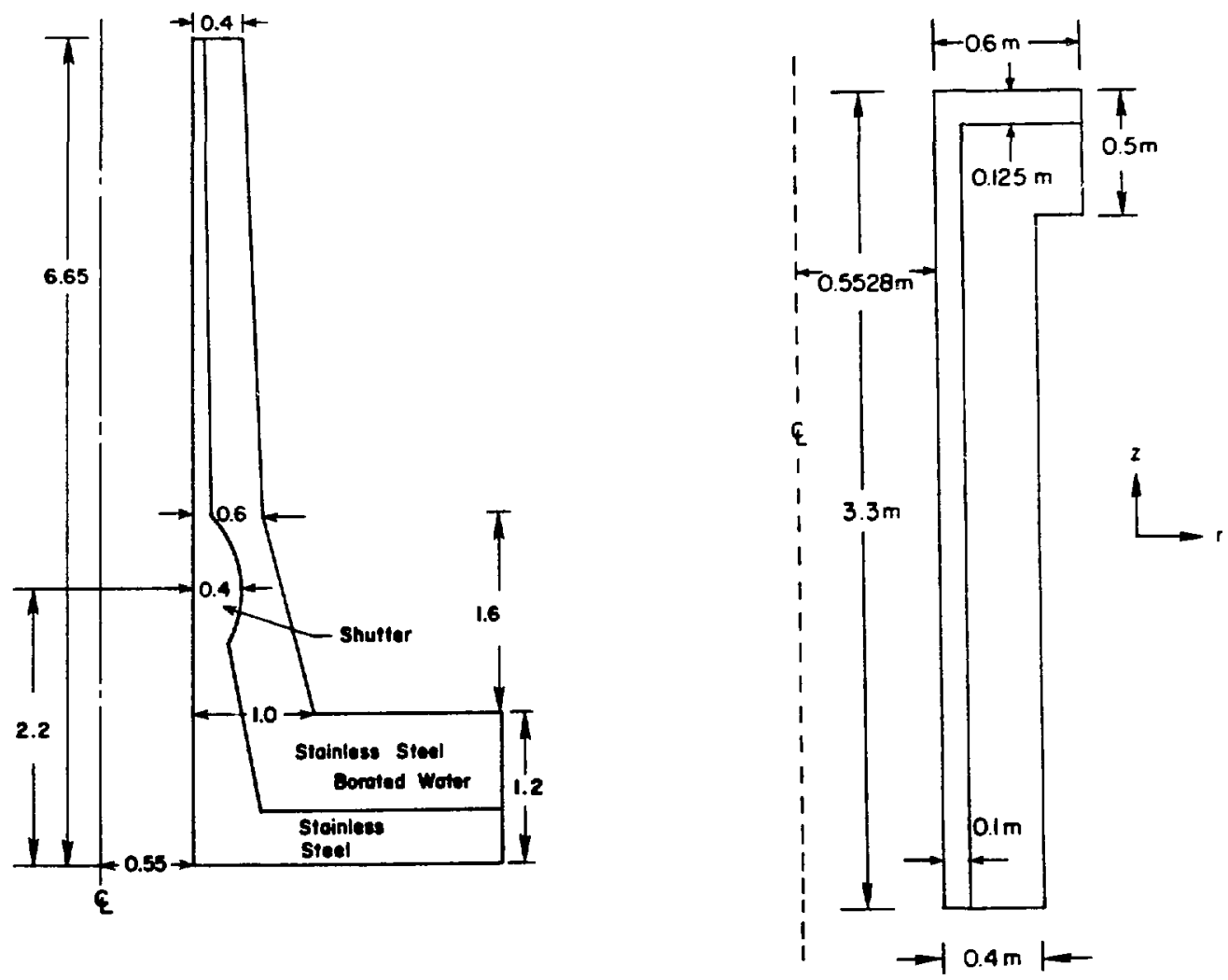

Fig. 26. NBI-duct model for TRIDENTCTR calculations. (DimenFig. 27. VP-duct model for TRIDENT-CTR sions in metres.)

The key input data to TRIDENT-CTR for both duct problems were the boundary (or surface) sources generated by MCNP. For the two duct problems several different space-angle distributions were provided with appropriate energy group weightings. The source was given as histogram distributions both angularly and spatially. The angular histogram was picked to match the highest order quadrature set used. Group-dependent quadrature sets were used in both problems, with an $S_{10} E Q N$ set being the highest order. ${ }^{9}$ This meant that spatial interpolations and angular interpolations in groups of a lower order quadrature 
had to be made. The spatial interpolations were fairly simple, and were made by assuming a linear variation of the source (for a given rle) between the centroids of the spatial histograms. The angular interpolations were quite a bit more complex and were made by overlaying the $\mathrm{S}_{10}$ quadrature structure on the other quadrature sets used. This was accomplished by associating an area on the unit sphere with each direction for each quadrature set. Then the intersecting areas of the different quadrature sets were used to make the interpolations. The actual mechanism used to perform this process was to calculate and use interpolation matrices such that

$$
\operatorname{BSN}(M)=\sum_{M M=1}^{15} \operatorname{SIN}(M, M M) * \operatorname{BS} 10(M M)
$$

where $\quad B S N=$ desired boundary source fo: direction $M$ of quadrature set $N$,

$$
\begin{aligned}
& \text { SIN = interpolation matrix for quadrature set } N \text {, } \\
& \text { BS10 = MCNP computed boundary source for } S_{10} .
\end{aligned}
$$

These interpolation matrices are given in Table III as a guide for future efforts of this nature.

The spatial and angular interpolations were performed in a preprocessor program that produced a binary source file compatible with TRIDENT-CTR. The bulk of the effort devoted to the TRIDENT-CTR calculations was spent in producing this processor.

\section{A. NBI-Duct Calculations}

A spatial mesh was formed on the model shown in Fig 26 by dividing the problem domain into 62 bands of triangles. The number of triangles in the bands varied from 25 to 44 , with a total of 1948 triangles. This meant for a 42- 
group, $\mathrm{P}_{3}$ calculation, 2454480 flux moments had to be calculated and stored $(3 \times 10 \times 42 \times 1948)$. The actual mesh is shown in Fig. 28.

The boundary conditions were set as follows:

(1) A reflective boundary was set along the $z$-axis at $r=0.0$ (left boundary).

(2) An internal boundary source was set along the z-axis at $r=0.533 \mathrm{~m}$ (right internal boundary source), directed into the shield.

(3) A bottom boundary source was set along the $r$-axis at $z=0.0$, directed into the shield but not the NBI duct (reproducing the source on the outboard shield).

(4) A vacuum boundary was set along the top and right surfaces of the problem domain.

The total source was normalized to a total of 1.0 neutron and 0.2414 gamma rays incident on both source surfaces of the problem. Of these, $95.504 \%$ were contained in the bottom boundary source (incident on the outboard shield) and $4.496 \%$ were contained in the right internal boundary source (incident on the duct wall). Also, separate group spectra, as determined from the MCNP calculations, were given for the bottom source and the right interior source. The actual normalizations were performed in the source preprocessor code. To obtain flux values normalized to a $2.4 \mathrm{MW} / \mathrm{m}^{2}$ wall loading, the values obtained with the 1.0 normalization were multiplied by $8.68 \times 10^{19}$. This renormalization factor is equal to the product of the MCNP absolute neutron flow at these boundaries times the total boundary source surface area.

A group-dependent set of $S_{N}$ orders was used for the problem, with an attempt to use the highest orders in those groups where the source was most anisotropic and strongest. The $\mathrm{S}_{\mathrm{N}}$ orders for each group are shown in Table IV.

The problem was run on the CDC-7600 at the NMFECC in approximately 70 minutes. Several regions of the problem were edited for comparison with the MCNP results. 


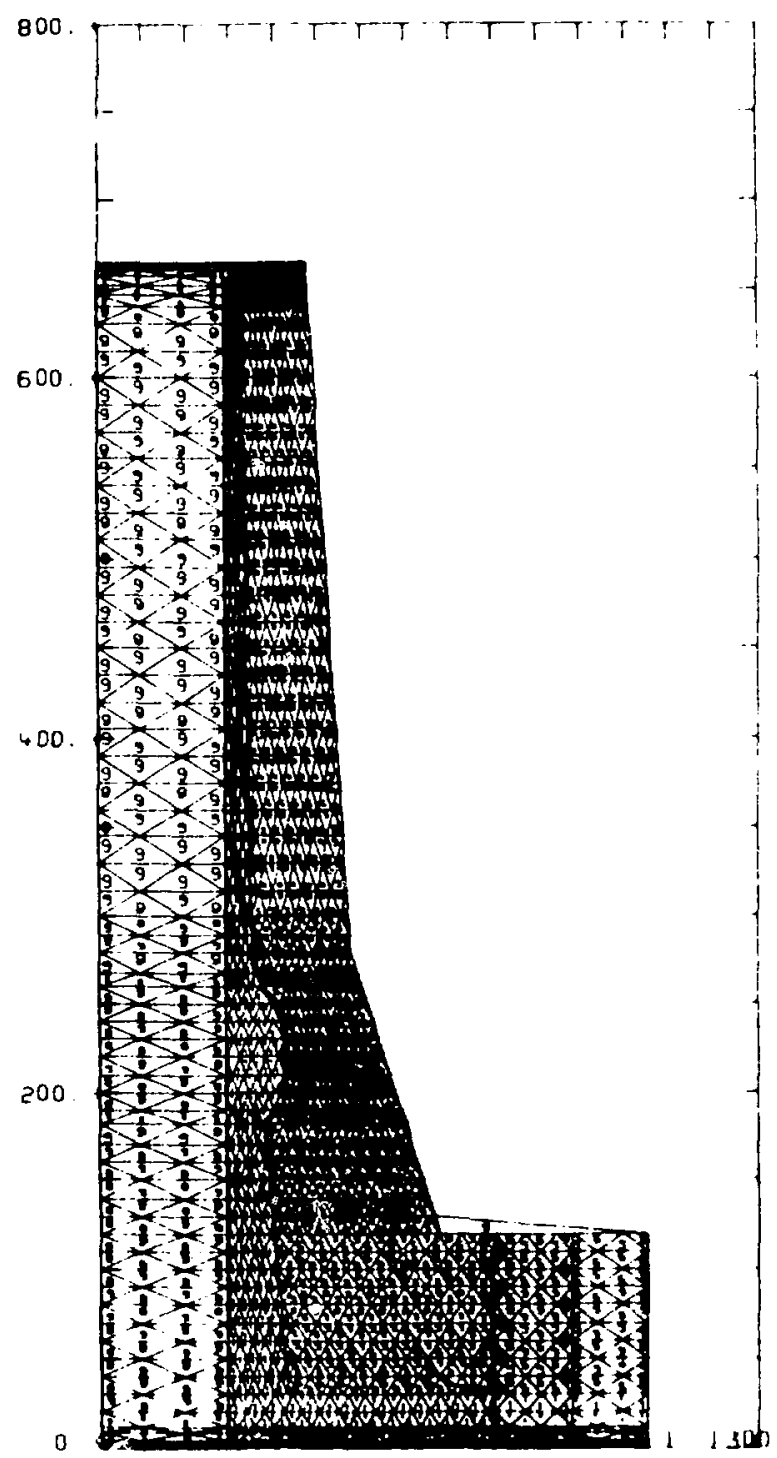

Fig. 28. TRIDENT-CTR spatial mesh for modeling the NBI duct, shielding, and cylindrical shutter. 
TABLE IV

GROUP DEPENDENCE OF QUADRATURE SETS FOR NBI-DUCT ANALYSIS

\begin{tabular}{|c|c|c|c|c|c|c|}
\hline Group & $\mathrm{S}_{\mathrm{N}}{ }^{\mathrm{a}}$ & Group & $\mathrm{S}_{\mathrm{N}}$ & Group & $\mathrm{S}_{\mathrm{N}}$ & \\
\hline 1 & 2 & 15 & 6 & 29 & 4 & \\
\hline 2 & 10 & 16 & 6 & $\begin{array}{l}30 \\
--\end{array}$ & $\begin{array}{c}4 \\
-\quad-\end{array}$ & - - \\
\hline 3 & 8 & 17 & 6 & 31 & 4 & G \\
\hline 4 & 6 & 18 & 4 & 32 & 4 & A \\
\hline 5 & 4 & 19 & 4 & 33 & 4 & M \\
\hline 6 & 4 & 20 & 4 & 34 & 4 & $M$ \\
\hline 7 & 4 & 21 & 4 & 35 & 4 & A \\
\hline 8 & 4 & 22 & 4 & 36 & 4 & \\
\hline 9 & 4 & 23 & 4 & 37 & 4 & $R$ \\
\hline 10 & 4 & 24 & 4 & 38 & 4 & A \\
\hline 11 & 4 & 25 & 4 & 39 & 6 & $\mathrm{X}$ \\
\hline 12 & 4 & 26 & 4 & 40 & 6 & $\mathrm{~S}$ \\
\hline 13 & 4 & 27 & 4 & 41 & 6 & \\
\hline 14 & 6 & 28 & 4 & 42 & 4 & \\
\hline
\end{tabular}

\footnotetext{
${ }^{\mathrm{a}_{2}}$ contains 4 directions.

$\mathrm{S}_{4}$ contains 12 directions.

$\mathrm{S}_{6}$ contains 24 directions.

$\mathrm{S}_{8}$ contains 40 directions.

$\mathrm{S}_{10}$ contains 60 directions.

${ }^{\text {All } 1 \text { quadrature sets are EQN sets. }} 9$
} 

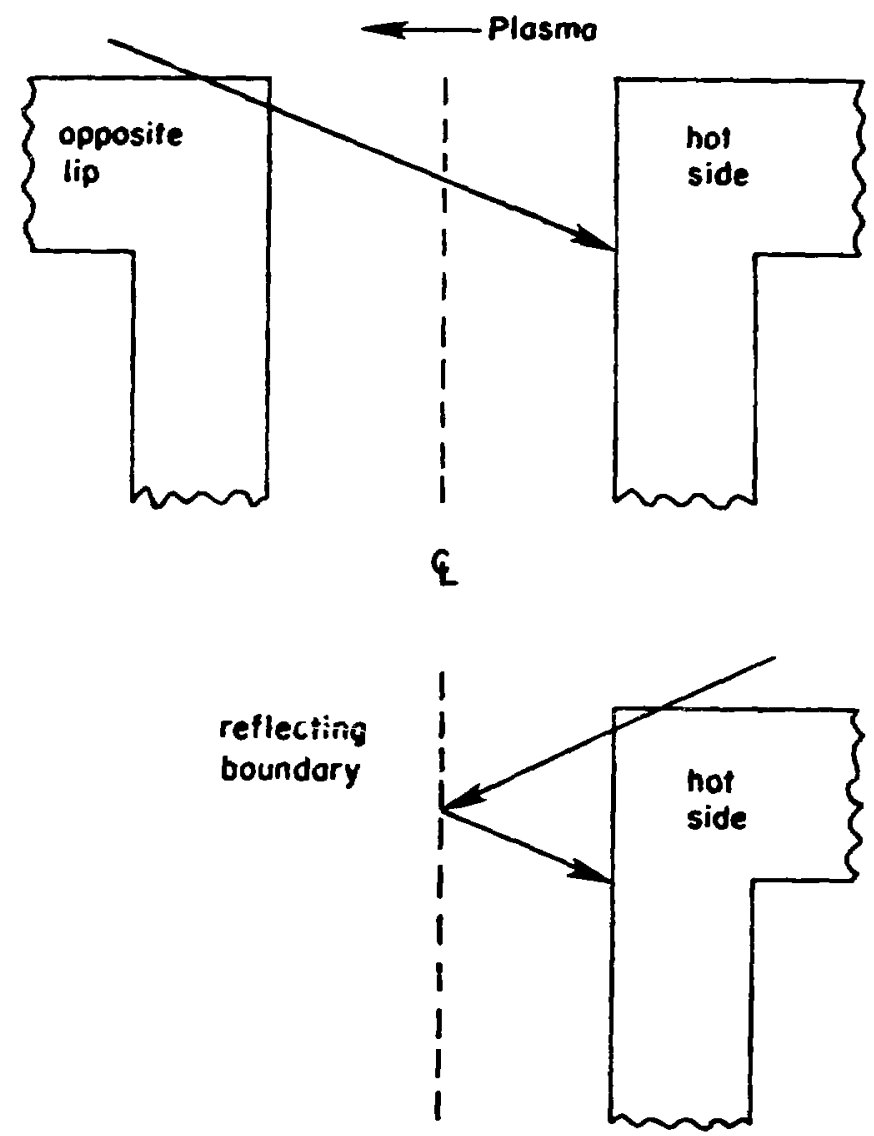

Fig. 29. TRIDENT-CTR VP-duct lip source illustrations.

\section{B. VP-Duct Calculations}

The vacuum-pumping (VP)-duct model shown in Fig. 27 was treated so as to yield conservative values (i.e., higher) for the the fluxes on the "hot" side of the VP duct (i.e., the side facing the plasma). This was accomplished by using an input source intensity on that wall based only on the neutrons hitting that wall in the MCNP calculations, and not on the duct average. Further, a lip source was constructed on the top boundary that, when coupled with a reflective boundary condition on the center line, represents the high-energy, anisotropic source on the lip opposite the "hot" wall. This was an attempt to account for those neutrons (especially the higher energy neutrons) that are incident on the lip opposite the "hot" wall, pass through the lip, and become incident on the "hot" wall. See Fig. 29. 
The TRIDENT-CTR spatial mesh for this problem was formed with 39 bands of triangles with between 35 to 51 triangles per band. A total of 1463 triangles were used, requiring the calculation of 1843380 flux moments. The mesh is shown in Fig. 30 .

The boundary conditions for the VP duct were set as follows.

(1) A reflective boundary was set along the $z$-axis at $r=0.0$ (left boundary.

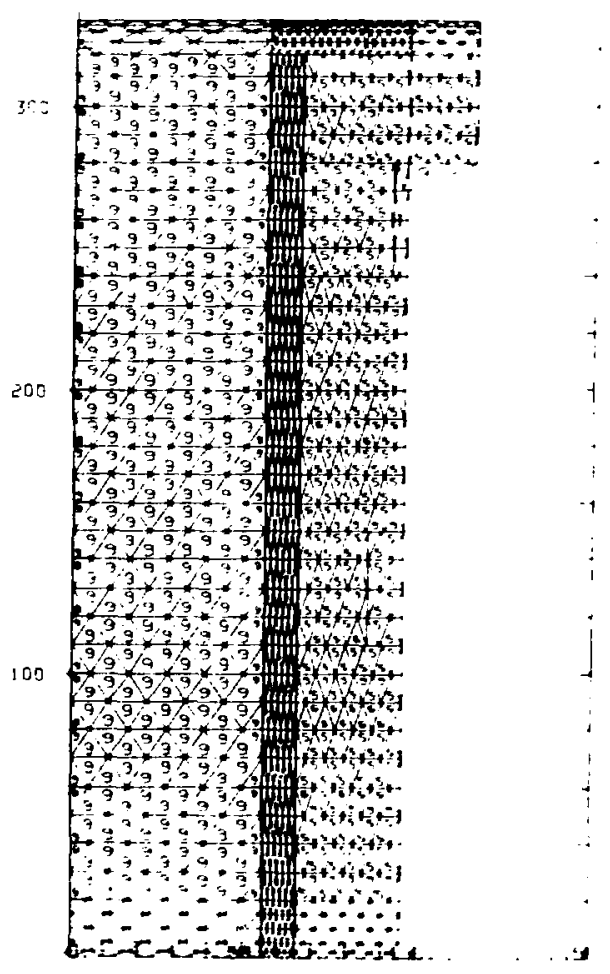

Fig. 30. TRIDENT-CTR spatial mesh for modeling the VP duct and shielding. 
(2) An internal boundary (right internal) source was set along the z-axis at $r=0.553 \mathrm{~m}$, directed into the shield.

(3) A top boundary source (lip source) was set along the $r$-axis at $z=3.3$ $m$, directed into the shield, but not down the VP duct.

(4) A vacuum boundary was set along the bottom and right surfaces of the problem domain.

The total source was normalized to 1.0 neutron and 0.1763 gamma rays. For this problem there were three different source spectra with three different angular distributions. The source spectra and angular distributions were obtained from the MCNP calculations. The orientation of each is shown in Fig. 31 and the amount of each in Table $V$. To obtain flux values normalized to a $2.4 \mathrm{MW} / \mathrm{m}^{2}$ wall loading in the plasma chamber, multiply the values obtained with the 1.0 neut ron normalization by $5.05 \times 10^{17}$. This renormalization factor is equal to the MCNP neutron flow at these boundaries times the total boundary source area.

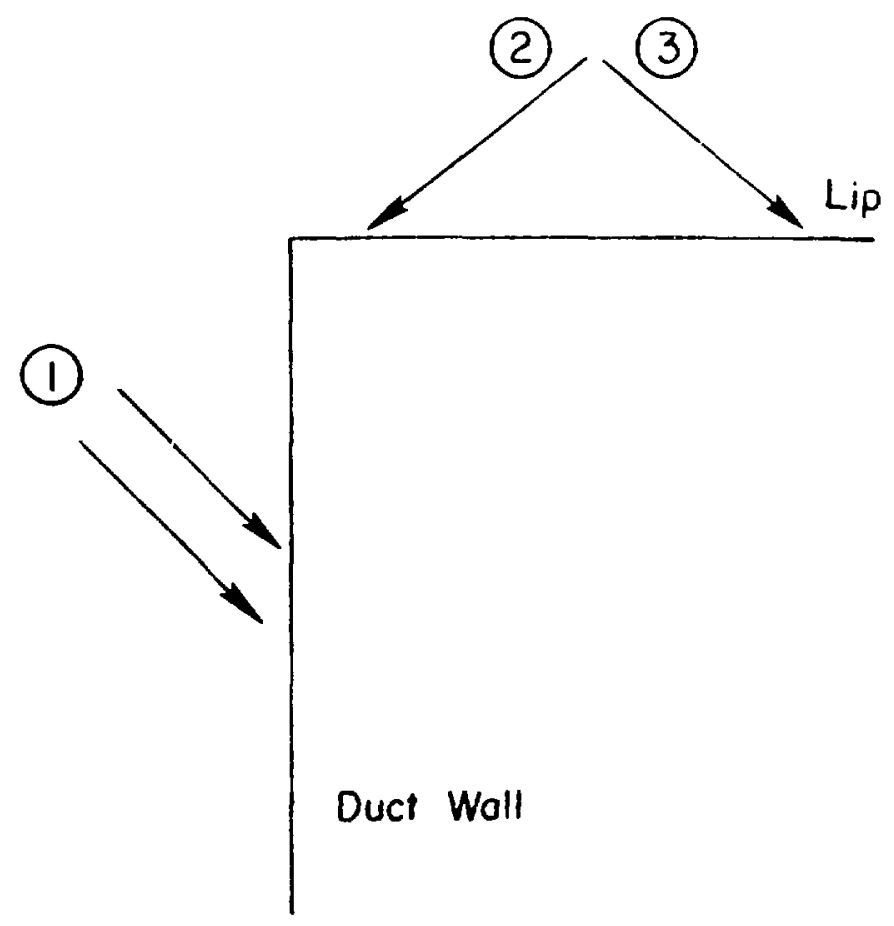

Fig. 31. TRIDENT-CTR VP-duct source orientation. 
TABLE V

VP-DUCT SOURCE NORMALIZATIONS

\begin{tabular}{|c|c|c|c|c|}
\hline & $\begin{array}{c}1 \\
\text { Down Duct }\end{array}$ & Inward on Lip $^{2}$ & Outward on Lip & Totals \\
\hline Neutrons & 0.2895 & 0.5194 & 0.1911 & 1.0 \\
\hline Gamma Rays & 0.0445 & 0.0885 & 0.0433 & 0.1753 \\
\hline
\end{tabular}

This problem required approximately 100 minutes of CDC-7600 time to run. The increased run times for this problem were a result of using a somewhat higher average $\mathrm{S}_{\mathrm{N}}$ order over all the energy groups. The group dependence of the $\mathrm{S}_{\mathrm{N}}$ orders used is shown in Table VI.

One of the interesting discoveries in this calculation was the emergence of negative scalar fluxes, generated by the inadequacy of the anisotropic scattering approximation in the $13.5-$ to $15 .{ }^{n}-\mathrm{MeV}$ energy group. This phenomenon had been discussed by Beranek and Conn ${ }^{10}$ in connection with time-dependent transport. It is believed that the highly anisotropic localized, 13.5- to 15.0MeV source at the top of the duct was responsible for the generation of these negative scalar fluxes. Refinement of the mesh in regions of negative flux had no effect, whereas switching to isotropic scattering order did eliminate the negative fluxes. For the final calculation a negative source fix-up was inserted for the appropriate energy group.

\section{DISCUSSION OF RESULTS}

Results from the MCNP and the hybrid MCNP/TRIDENT-CTR calculations for the NBI and VP ducts are presented in this section, along with a comparison of the results between the two calculational methods. The results from the intermediate calculations were presented in Sections III.B, III.C, and III.D, where they were used to clarify the calculational approach that led to the NBI- and VP-duct shield calculations. Unless otherwise indicated, the data presented in this section are normalized to a neutron-flow incident on the torus shield from neutrons with energies between 13.5 and $15.0 \mathrm{MeV}$ of $2.4 \mathrm{MW} / \mathrm{m}^{2}$. 
TABLE VI

GROUP DEPENDENCE OF QUADRATURE SETS FOR VP-DUCT ANALYSIS

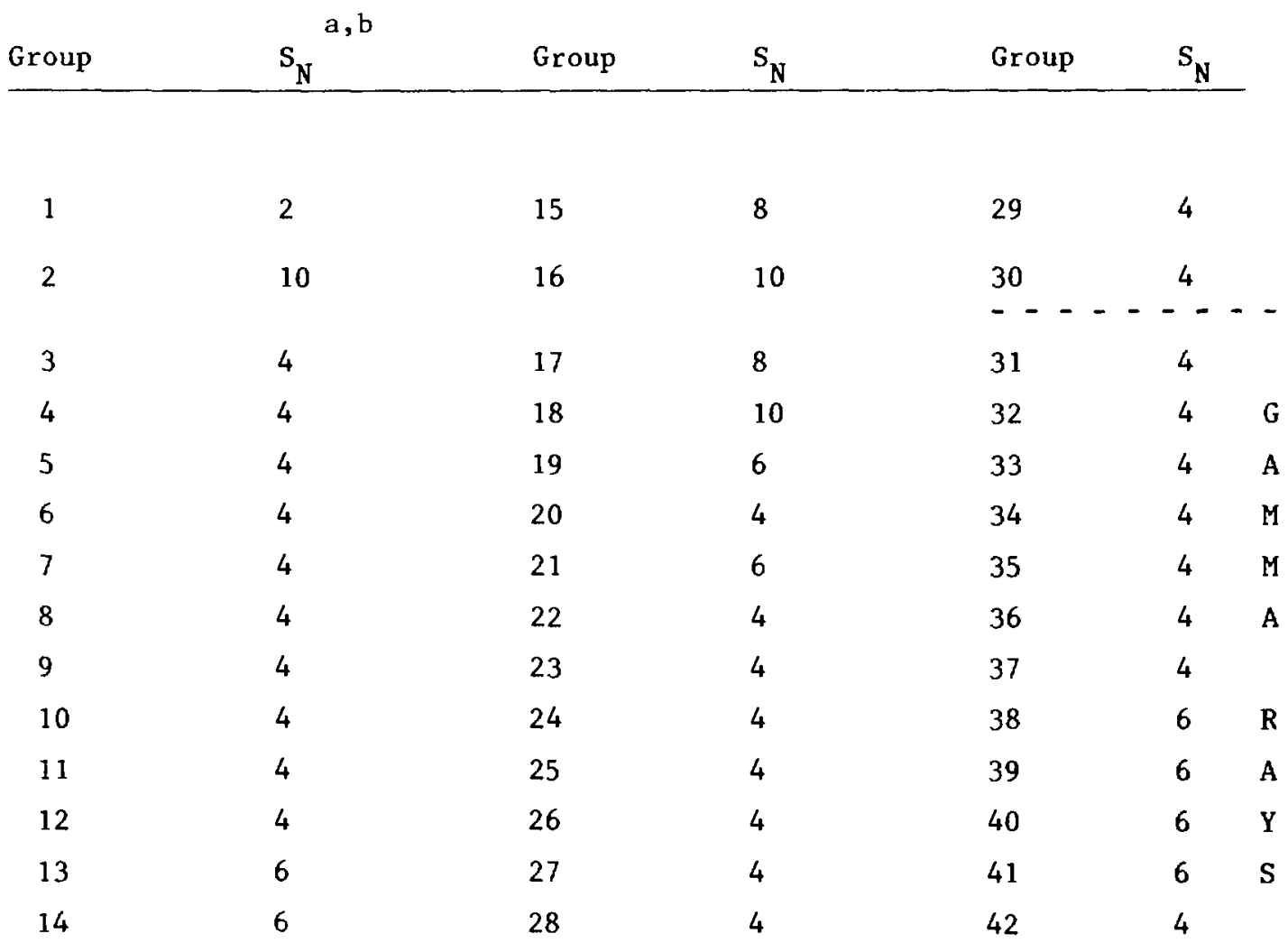

\footnotetext{
${ }_{\mathrm{S}_{2}}$ contains 4 directions.

$\mathrm{S}_{4}$ contains 12 directions.

$\mathrm{S}_{6}$ contains 24 directions.

$\mathrm{S}_{8}$ contains 40 directions.

$S_{10}$ contains 60 directions.

${ }^{\mathrm{b}} \mathrm{A1} 1$ quadrature sets are EQN sets. ${ }^{9}$
} 
A. NBI Duct

1. MCNP Results. Information regarding the neutron flux in the NBI duct is contained in Figs. 32 through 34. Figure 32 shows a comparison of the total neutron flux profile down the duct to the flux resulting from the first flight, in the NBI duct, of neutrons direct from the torus. The difference between these two histograms represents the flux resulting from neutrons which have scattered from the duct walls. The contribution to the total flux from the first flight of the torus neutrons is about $50 \%$ at the duct mouth, $15 \%$ at $3.0 \mathrm{~m}$, and $25 \%$ at the end of the duct. The large decrease in the middle of the duct results from the scattered contribution to the total flux going through a maximum. The scattered contribution decreases at the end of the duct because only the inboard surfaces of the duct walls can contribute. In this calculation no scattered neutrons return from the NBI chamber. Figure 33 presents the total neutron flux profile down the duct, plus a three-energy-group breakdown of the total flux. This figure illustrates the spatial dependence of the energy distribution in the duct and also the large contribution from neutrons with $\mathrm{E}<0.5 \mathrm{MeV}$. Also, it shows in detail the effect of duct-wall scattering on the neutron energy distribution. A detailed neutron flux spectrum averaged over the last $0.04 \mathrm{~m}$ of the duct is presented in Fig. 34. Integrating this spectrum over energy yields a total flux of $9.41 \times 10^{12}$ neutrons $/ \mathrm{cm}^{2} \mathrm{~s}$. Upon comparison with Fig. 9 , which is the neutron flux spectrum at the torus outboard shield first wall, it is apparent that the 13.5 to $15.0 \mathrm{MeV}$ neutrons are greater contributors to the total flux at the end of the duct $(\sim 21 \%)$ than at the torus outboard shield first wall ( $\sim 11 \%$ ). However, when one compares the flow of neutrons (in the outboard direction) across the torus outboard shield first wall to that at the end of the duct the contribution of the high-energy neutrons to the total is similar; i.e., $\sim 25 \%$. These observations relating to the flux and flow at the torus outboard shield first wall have been discussed earlier and result, in part, because the flow is only for neutrons traveling in the outboard direction, whereas the flux takes into account neutrons crossing the surface with both inboard and outboard directions.

Fractional errors for the fluxes in Fig. 32 vary from less than $1 \%$ to $5 \%$. The groupwise fluxes of Fig. 33 all have fractional errors of less than $10 \%$ with the majority being less than $5 \%$. The total (energy integrated) flux represented by Fig. 34 has a fractional error of $3 \%$. 


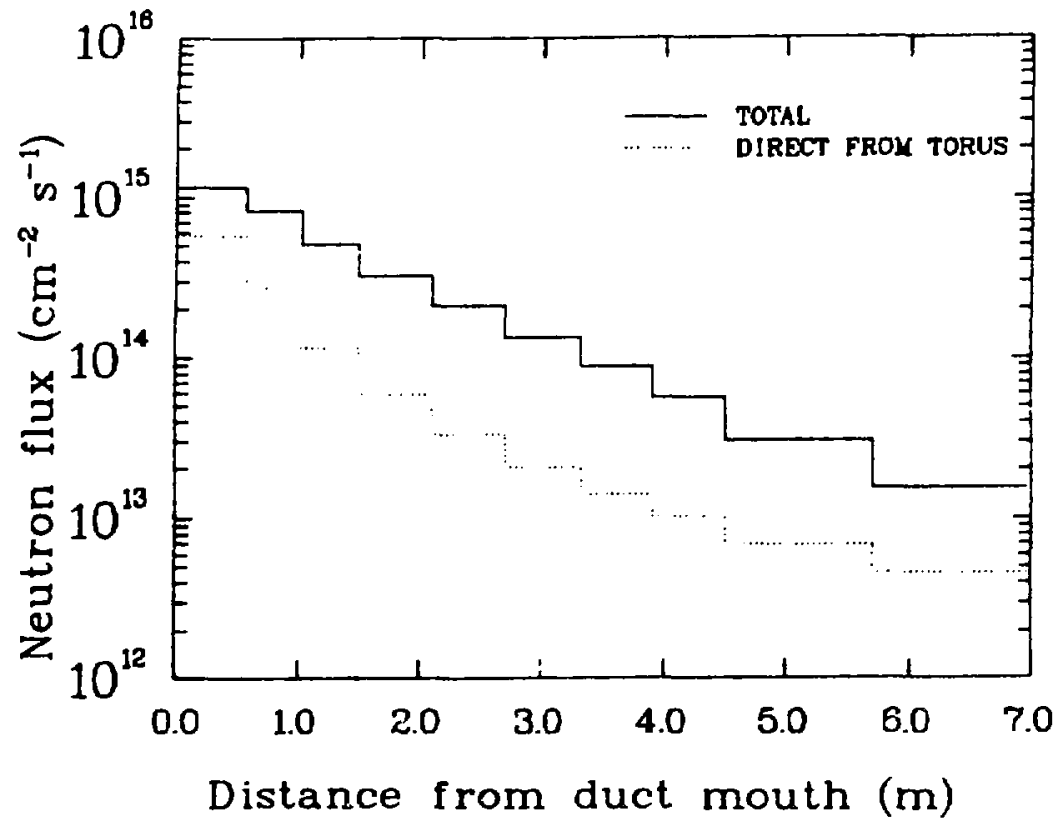

Fig. 32. Comparison of the total neutron flux down the NBI duct to the flux from the first flight in the NBI duct of neutrons direct from the torus as determined from MCNP calculations.

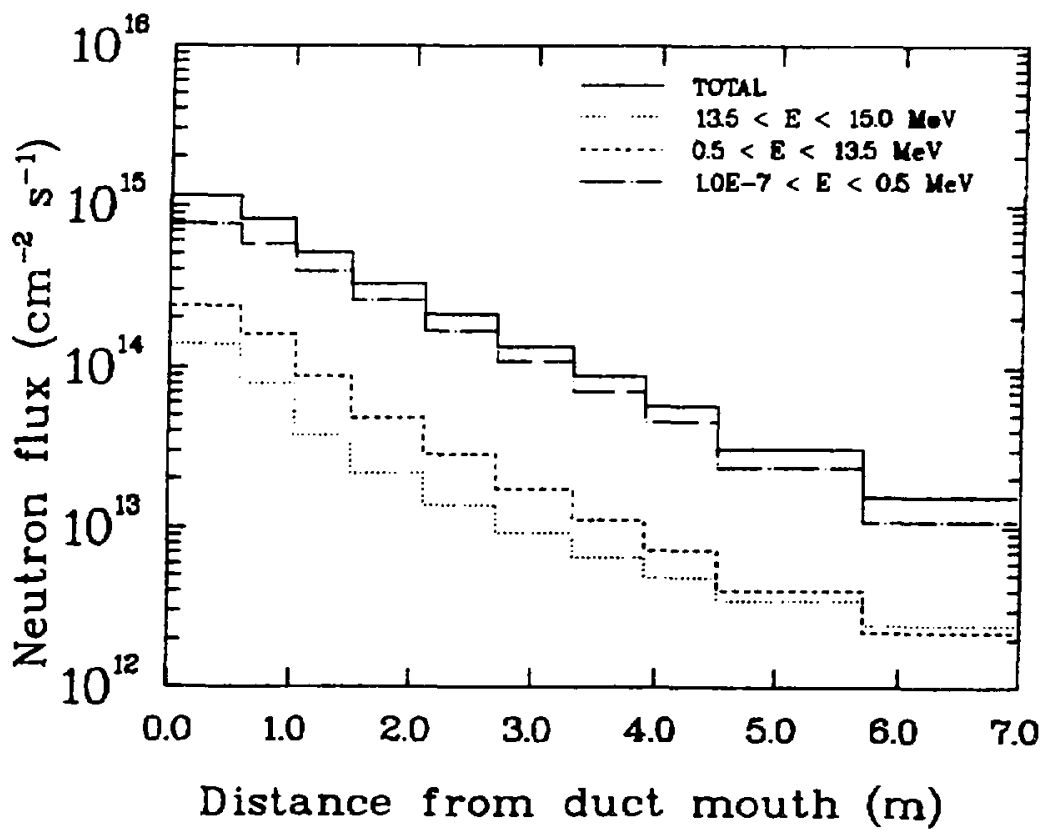

Fig. 33. Energy-dependent neutron flux profiles down the NBI duct as determined by MCNP. 


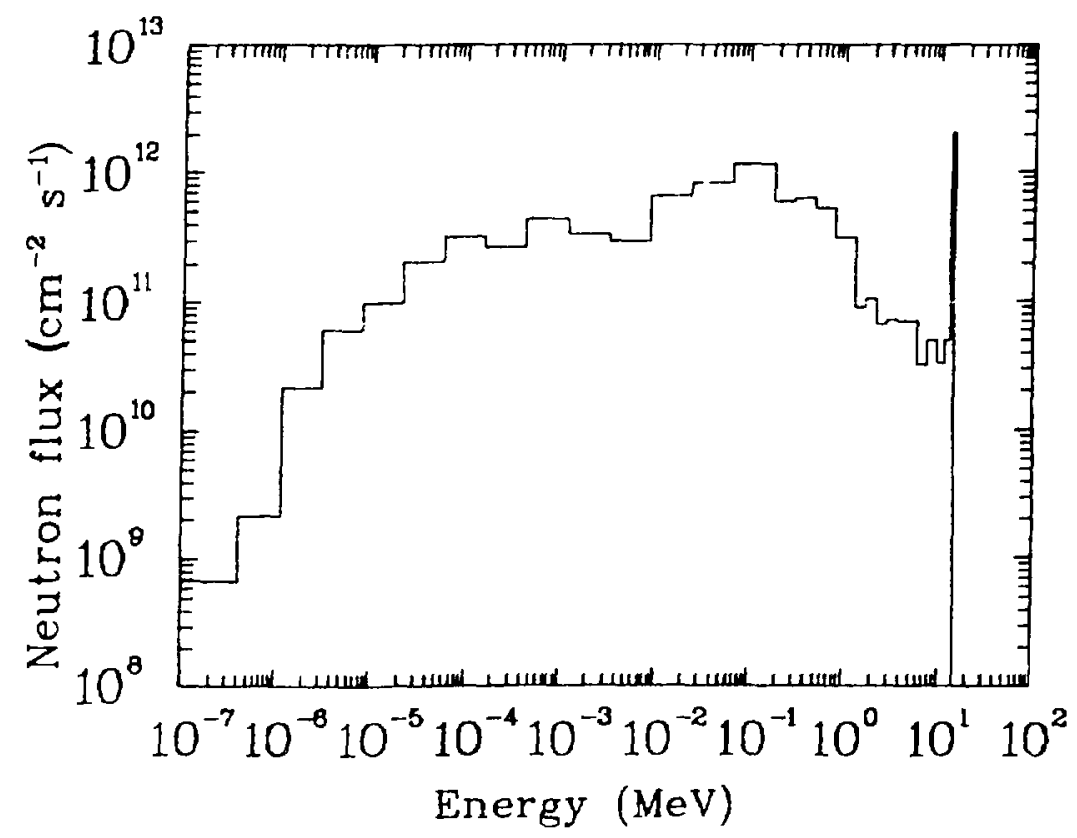

Fig. 34. Average neutron spectrum in the last $0.04 \mathrm{~m}$ of the NBI duct as determined by MCNP.

An important consequence of the NBI-duct shield calculations is the information obtained regarding the neutrons and gamma rays which enter the NBI chamber. Figures 35 through 38 contain this information. The data represent the average values over the duct cross-sectional area; i.e., $0.8 \times 1.2 \mathrm{~m}$. The angular distribution and energy spectrum of the gamma rays flowing from the NBI duct into the NBI chamber are presented in Figs. 35 and 36 , respectively. Both figures show the total flow (i.e., gamma rays originating in the NBI - duct shield plus the torus inboard and outboard shields as well as the flow from the NBI shield only. The angular distribution is sharply peaked in the forward direction (i.e., along the duct axis toward the NBI chamber), and approximately $65 \%$ of the gamma rays entering the NBI chamber enter with cosine(theta) $>0.96$. The last three cosine(theta) breakpoints are at $0.9,0.96$ and 0.99 with 1.0 the upper limit of the last bin. Gamma rays from the torus shields predominate 


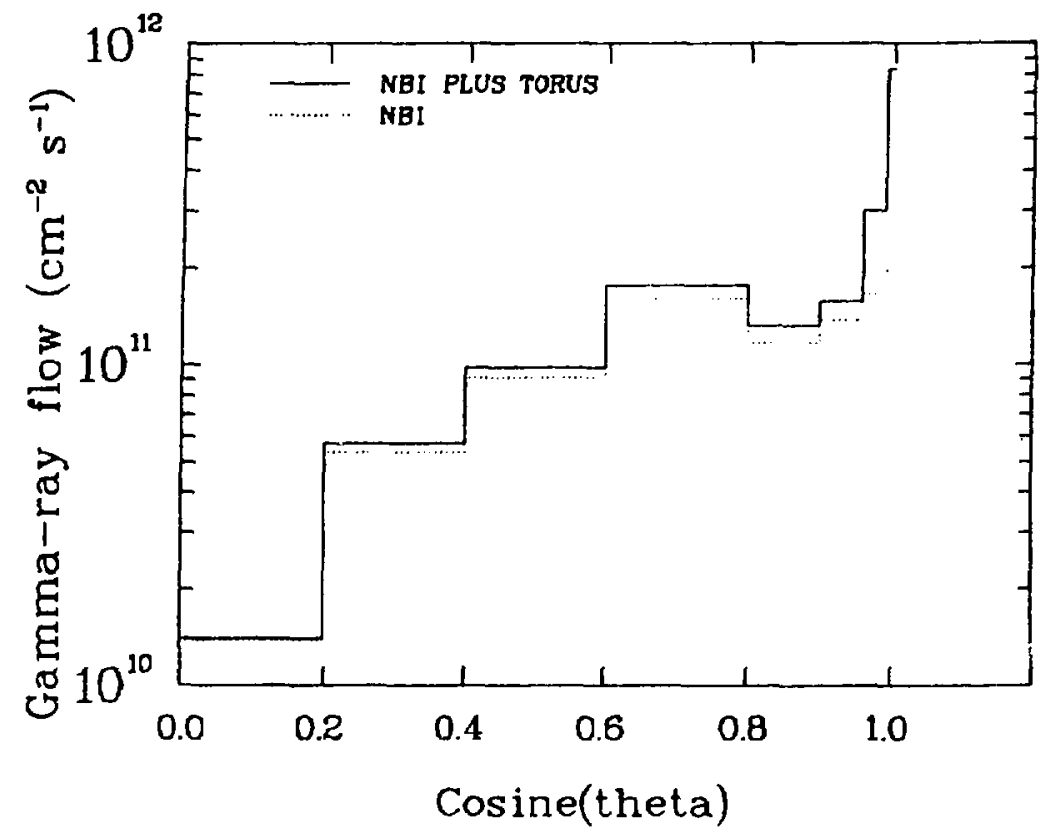

Fig. 35. Angular distribution of gamma rays flowing from the NBI duct into the NBI chamber for gamma rays originating in the NBIduct shield, and the sum for those originating in both the torus inboard and outboard shields plus the NBI-duct shield. (MCNP results.)

for cosine(theta) $>0.96$, which is reasonable because the torus gamma rays are effectively a source over the opposite end (the mouth) of the duct. Conversely, the duct walls are parallel to the duct axis, thereby requiring the gamma rays originating in the duct walls to enter the duct in a direction nearly parallel to the duct walls if they are to contribute to cosine(theta) bins with values greater than 0.96. The energy distributions of gamma rays entering the NBI chamber for the same sources of gamma rays are presented in Fig. 36, where the two spectra are seen to be very sinilar. Of the total gamma rays entering the NBI chamber, $53 \%$ originate in the NBI-duct shielding and $47 \%$ in the torus shields.

The reader is cautioned in using these gamma-ray data on a detailed basis because some of the individual flow bins have fractional errors exceeding $10 \%$. Until better data become available, the authors are of the opinion that it is of value to provide this information, even with the realization that some parts of the histogram are statistically suspect. 


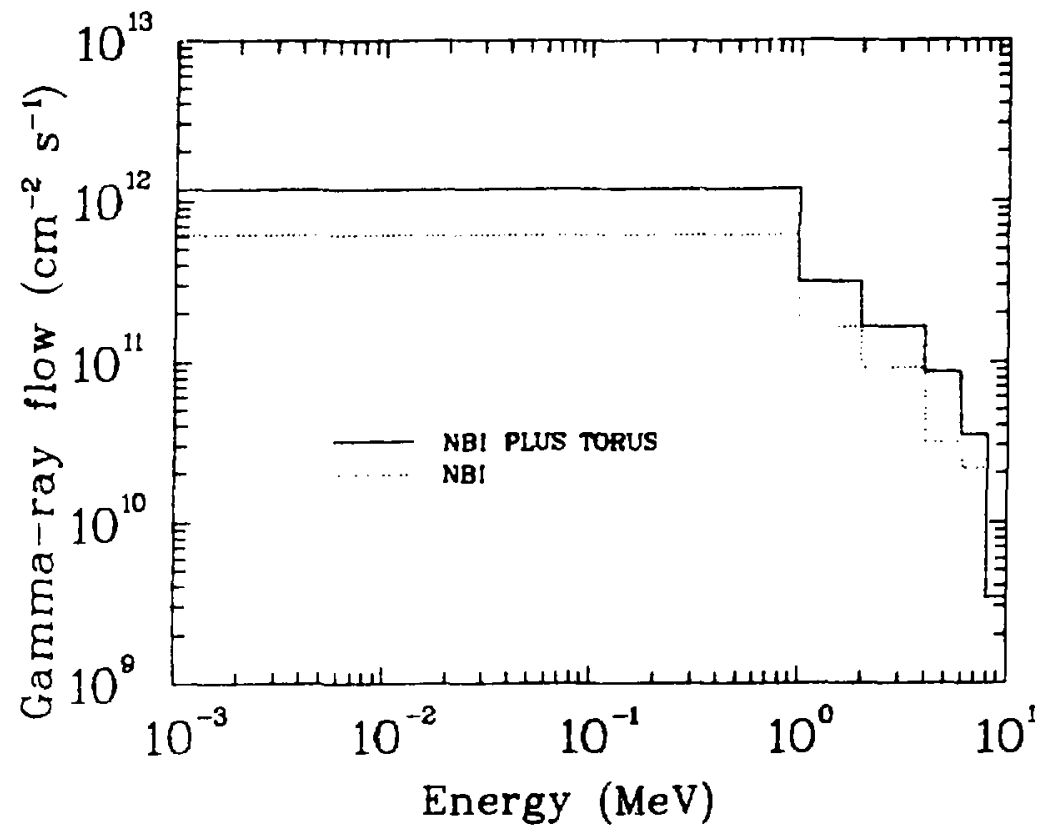

Fig. 36. Energy spectrum of gamma rays entering the NBI chamber from the NBI duct for gamma rays originating in the NBI-duct shield as well as the sum of those originating in the torus inboard and outboard shields plus the NBI duct shield. (MCNP results).

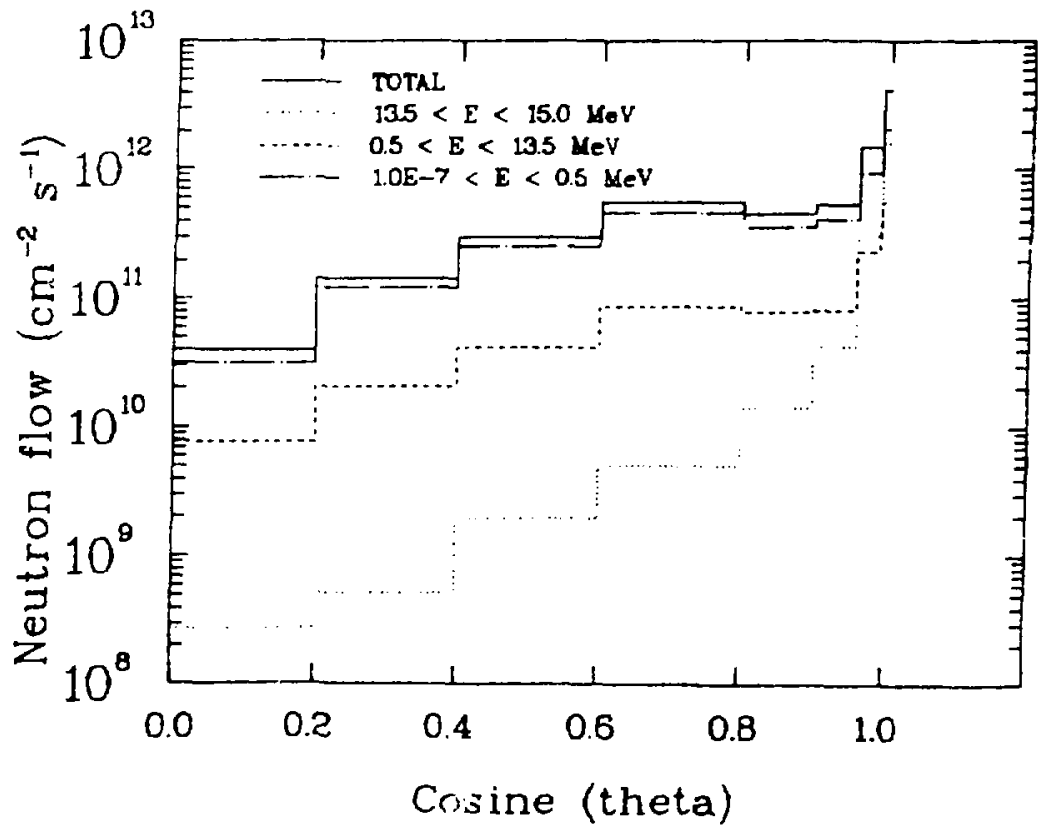

Fig. 37. Energy-dependent angular distribution of neutrons entering the NBI chamber from the NBI duct. Theta is the angle between the duct axis and the neutron direction. (MCNP results.) 


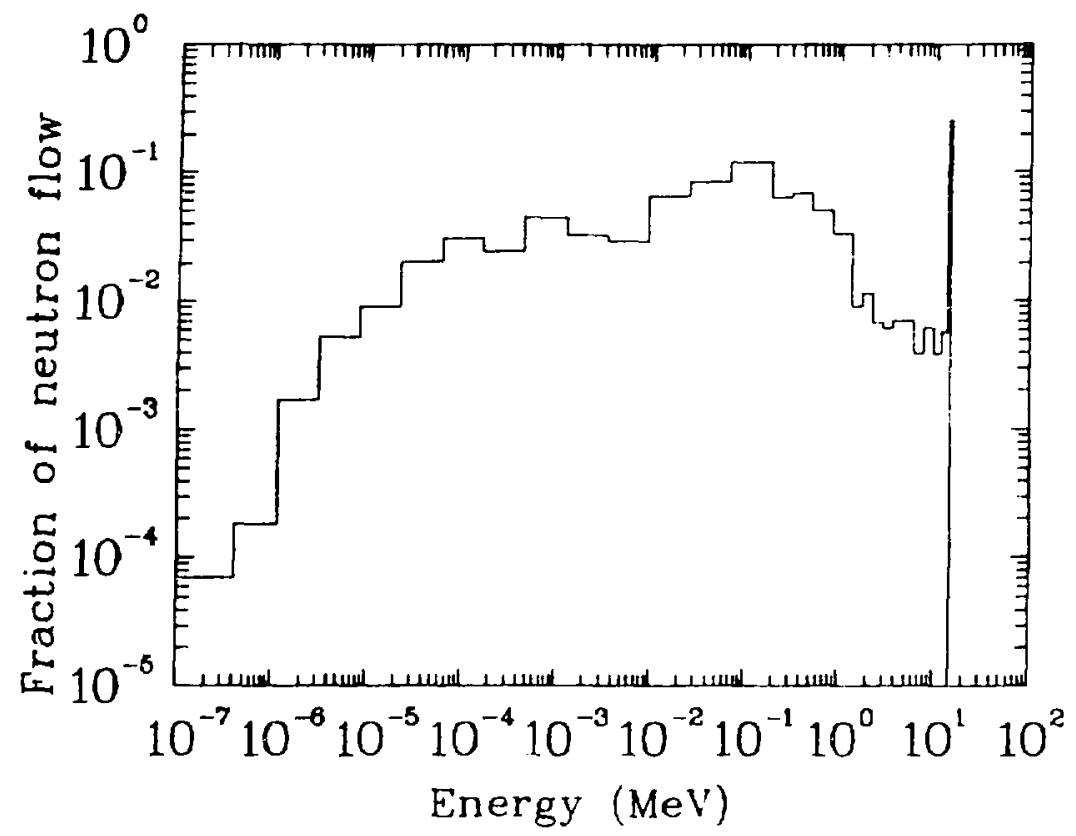

Fig. 38. Spectrum of neutrons entering the NBI chamber from the NBI duct as determined by MCNP. Normalized to one neutron entering the chamber.

Figures 37 and 38 present the energy-dependent angular distribution and spectrum, respectively, of neutrons flowing from the NBI duct into the NBI chamber. The angular distribution data clearly show that the forward peaking increases with increasing neutron energy. This is to be expected because the higher the neutron energy the fewer collisions a neutron has undergone and thus, its angular distribution more closely approximates that resulting from neutrons which travel from the toroidal plasma chamber to the NBI chamber without an interaction in the NBI-duit walls. From the histogram for the total flow (energy integrated), it can be determined that $\sim 75 \%$ of the neutrons enter the chamber with values of cosine(theta) greater than 0.96 . The energyintegrated neutron flow into the NBI chamber is $7.74 \times 10^{12}$ neutrons $/ \mathrm{cm}^{2} \mathrm{~s}$.

The neutron flow spectrum of Fig. 38 is very similar in shape to the average neutron flux spectrum in the last $0.04 \mathrm{~m}$ of the duct as shown in Fig. 34 . The magnitude of the flux of Fig. 34 in the energy range 13.5 to $15.0 \mathrm{MeV}$ is $2.01 \times 10^{12}$ neutrons $/ \mathrm{cm}^{2} \mathrm{~s}(\sim 21 \%$ of the total), whereas the magnitude of the 
flow in this same energy interval is $1.99 \times 10^{12}$ neutrons $/ \mathrm{cm}^{2} \mathrm{~s}(\sim 26 \%$ of the total). Because all of these neutrons are traveling toward the NBI chamber, then their average cosine relative to the NBI-duct axis would be cosine(theta) $=\left(1.99 \times 10^{12} / 2.01 \times 10^{12}\right)=0.99$. Looking back at the angular distribution data for this energy range as given in Fig. 37 one indeed finds that the average cosine(theta) value is 0.99 . The fractional error of the total spectrum is $3.5 \%$ and the binwise fractional errors over the major contributing portion of the spectrum (i.e., $3.06 \times 10^{-6}$ to $1.353 \mathrm{MeV}$ ) vary from 10 to $15 \%$, and that for the $13.5-$ to $15.0-\mathrm{MeV}$ bin is $9 \%$. Errors in the remaining energy bins are higher than $15 \%$.

These data on the flow of neutrons and gamma rays entering the NBI chamber are of importance in evaluating the potential for radiation damage in the chamber. The total number of neutrons entering the chamber would be $\left(7.74 \times 10^{12}\right.$ neutrons $\left./ \mathrm{cm}^{2} \mathrm{~s}\right)\left(80 \times 120 \mathrm{~cm}^{2}\right)=7.43 \times 10^{16}$ neutrons $/ \mathrm{s}$, or $1.91 \times 10^{16}$ neutrons/s with energies between 13.5 and $15.0 \mathrm{MeV}$. Components of the NBI directly in Iine with the duct would therefore be subjected to a very intense beam of high-energy neutrons, which might affect the planned operation of the NBI due to radiation damage or cryopanel nuclear heating. The values just cited were for an open duct. Use of a $0.6-\mathrm{m}$-thick stainless steel sibutter shield might reduce the $13.5-$ to $15.0-\mathrm{MeV}$ flux by a factor of approximately $\mathrm{e}^{-\mathrm{N} \sigma_{\mathrm{T}} t}=\exp (-0.0858 \mathrm{x}$ $2.5 \times 60)=2.6 \times 10^{-6}$. However, this would still result in a high-energy neutron leakage into the NBI chamber of $\sim 5 \times 10^{10}$ neutrons/s, which still appears to be quite high. Although it is beyond the scope of this effort to assess the po-natial damage in the NBI chamber caused by this intense beam of neutrons, it is the opinion of the authors that the radiation effects questions must be resolved soon because they might easily prove to be a limiting factor with respect to the NBI concept.

2. TRIDENT-CTR Results. TRIDEITT-CTR results presented here are the end prod:st of the hybrid MCNP/TRIDENT-CTR calculations. These calculations yielded point-wise neutron and gamma-ray fluxes from which it is possible to generate various neutron and gamma-ray responses such as heating, exposure rate, etc. Figures 39 and 40 contain neutron flux profiles over the entire geometry and a blow-up in the vicinity of the shutter. Neutron, gamma-ray, and total heating profiles for these geometries are presented in Figs. 41 through 46. 


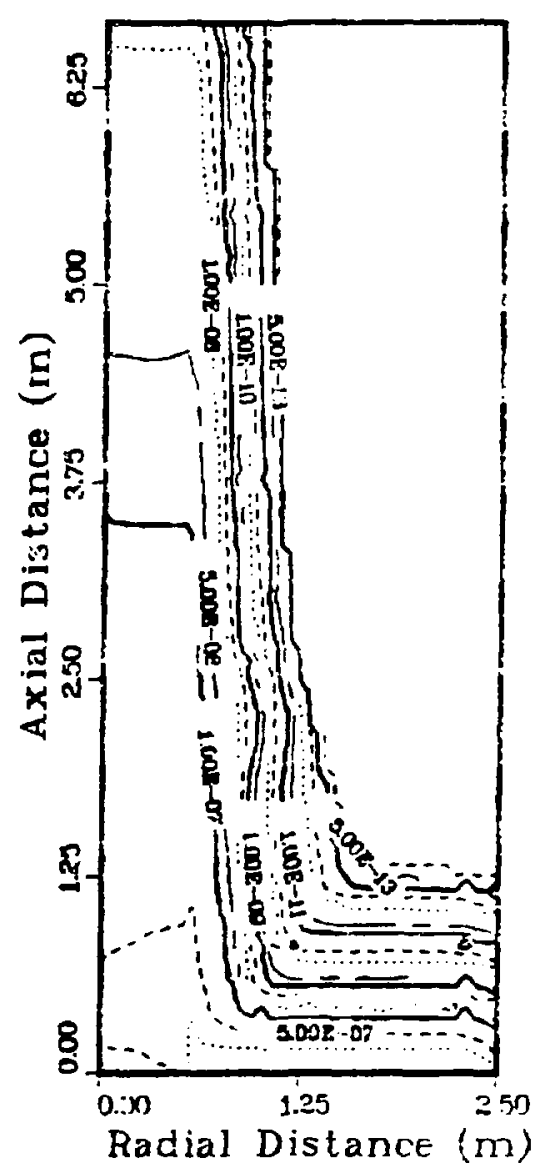

Fig. 39. TRIDENT-CTR total neutron flux $\left(\mathrm{cm}^{-2} s_{9}^{-i}\right)$ in the NBI-duct shield. Multiply values by $8.68 \times 10^{19}$. See Fig. 26 for geometric model. 


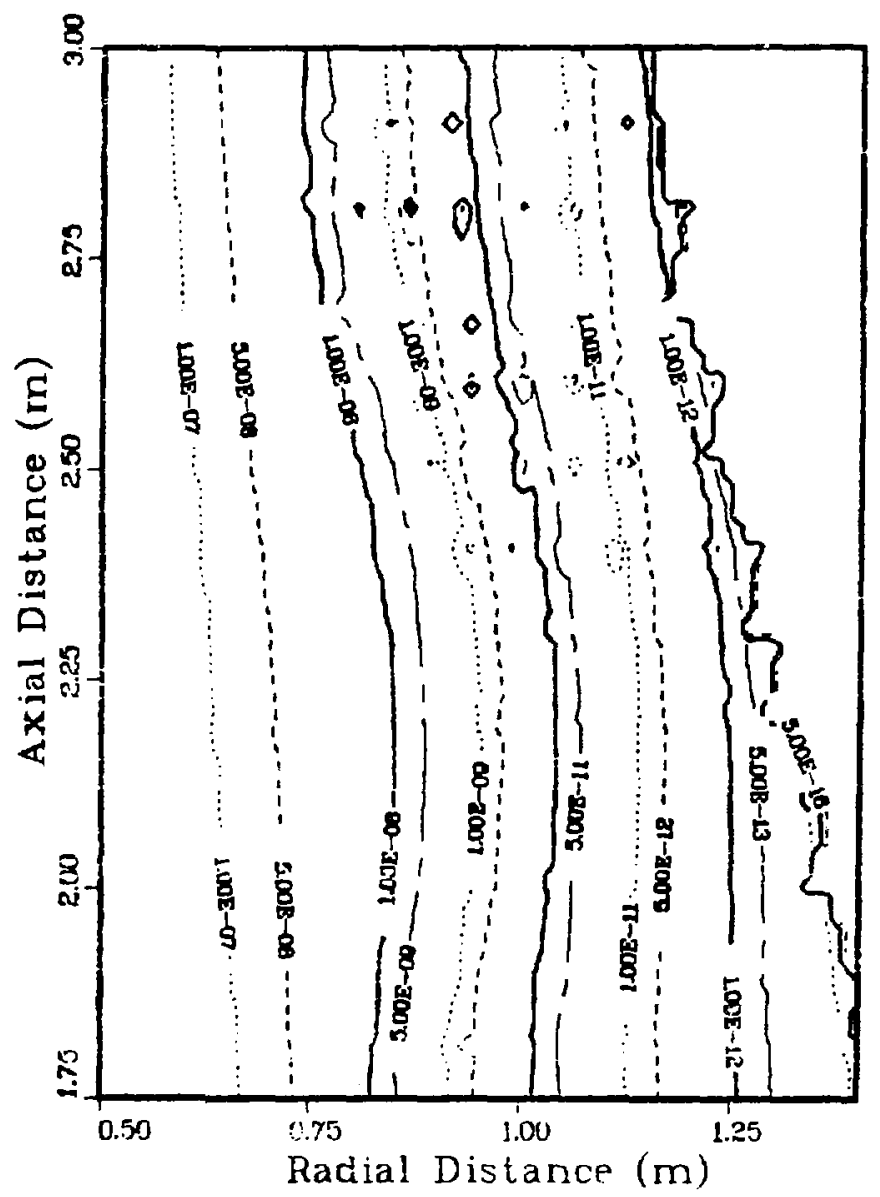

Fig. 40. TRIDENT-CTR total neutron flyg $\left(\mathrm{cm}^{2} \mathrm{~s}^{-1}\right)$ at the NBI-duct shutter. Multiply values by $8.68 \times 10^{19}$. See Fig. 26 for geometric model. 


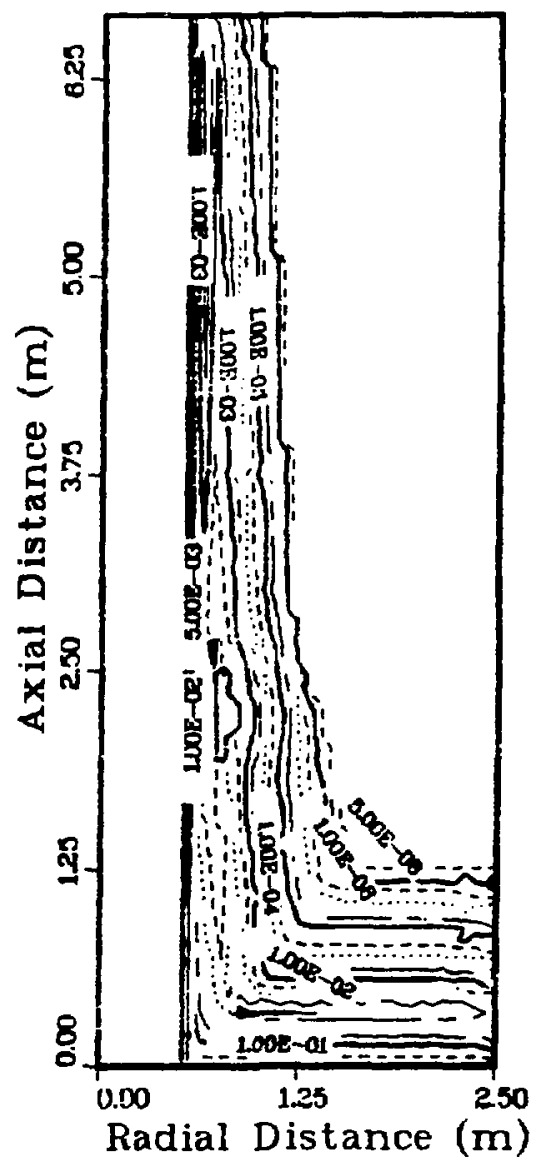

Fig. 41. TRIDENT-CTR neutron heating $\left(\mathrm{MW} / \mathrm{m}^{3}\right)$ in the NBI-duct shield. See Fig. 26 for geometric model. 


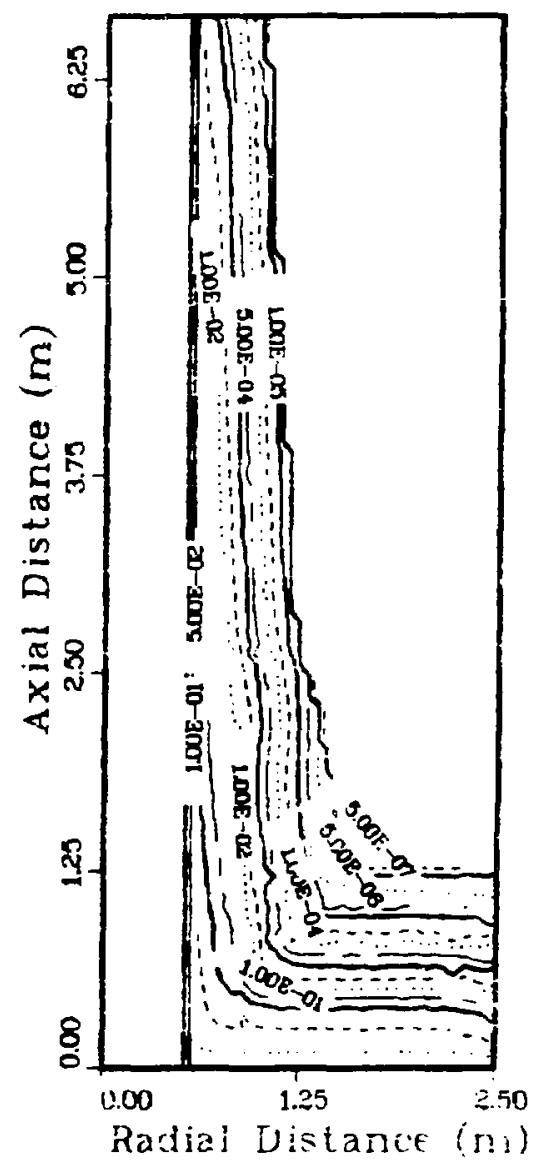

Fig. 42. TRIDENT-CTR gamma-ray heating $\left(\mathrm{MW} / \mathrm{m}^{3}\right)$ in the NBI-duct shield. See Fig. 26 for geometric mode1. 


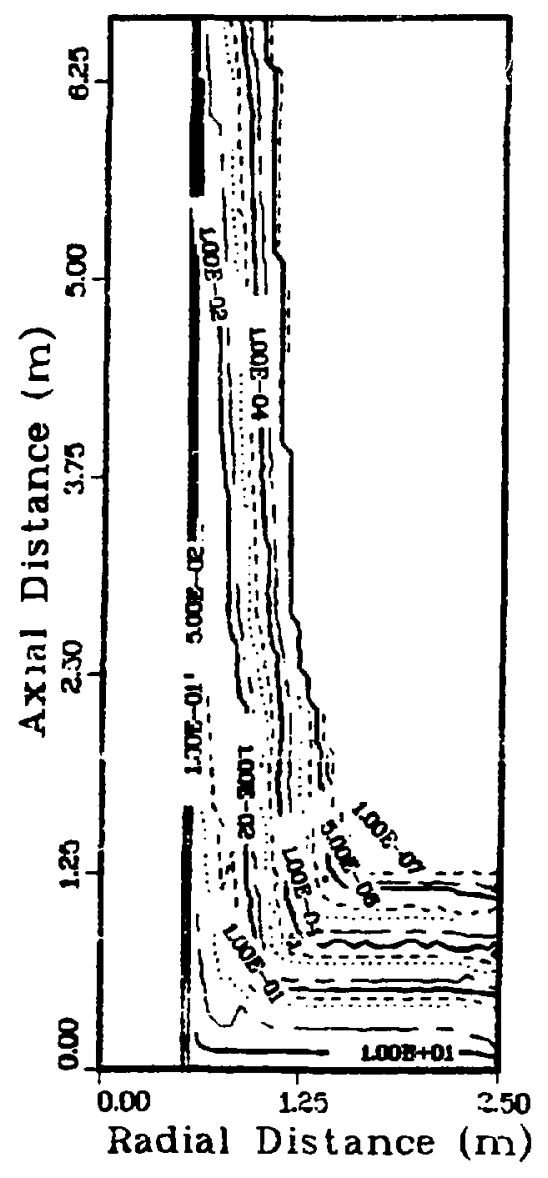

Fig. 43. TRIDEHT-CTR total (neutron plus gamma-ray) heating $\left(\mathrm{MW} / \mathrm{m}^{3}\right)$ in the NBI-duct shield. See Fig. 26 for geometric model. 


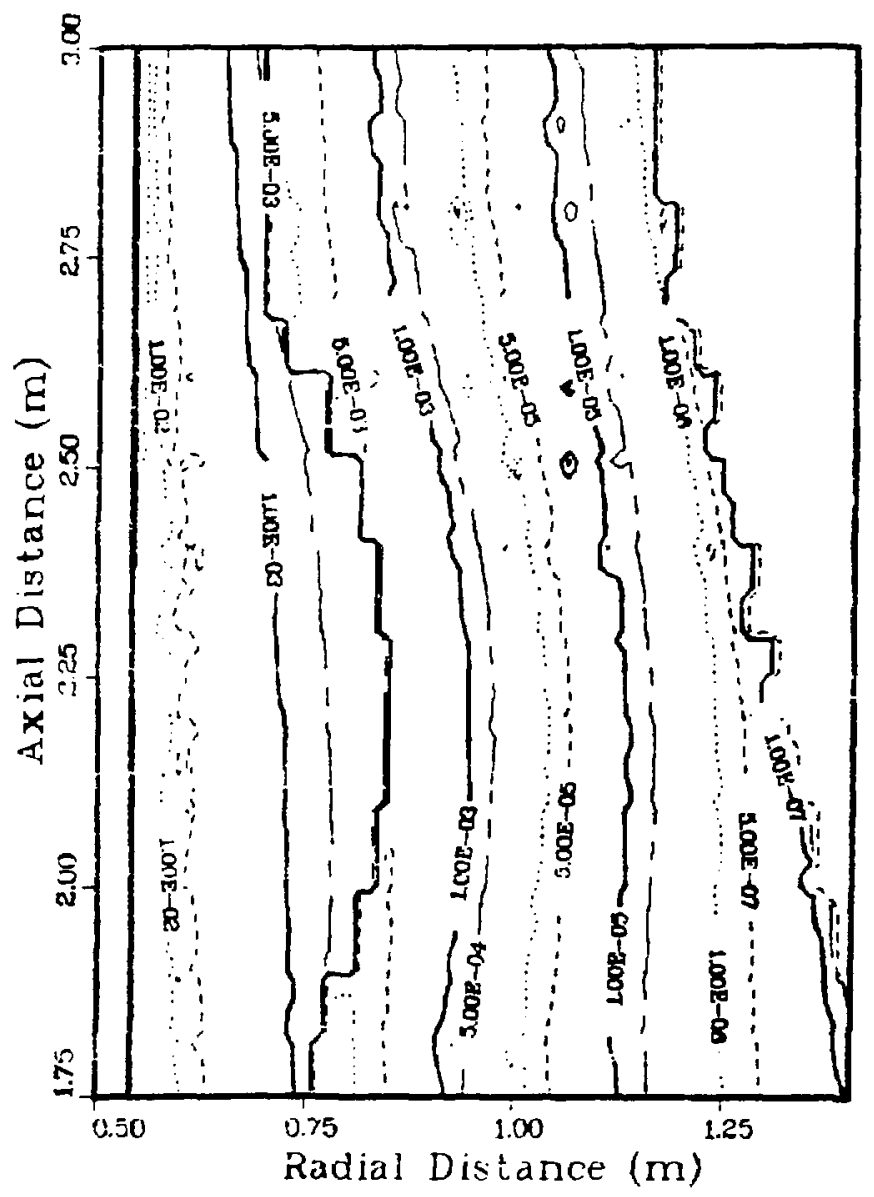

Fig. 44. TRIDENT-CTR neutron heating $\left(\mathrm{MW} / \mathrm{m}^{3}\right)$ at the NBI-duct shutter. See Fig. 26 for geometric model. 


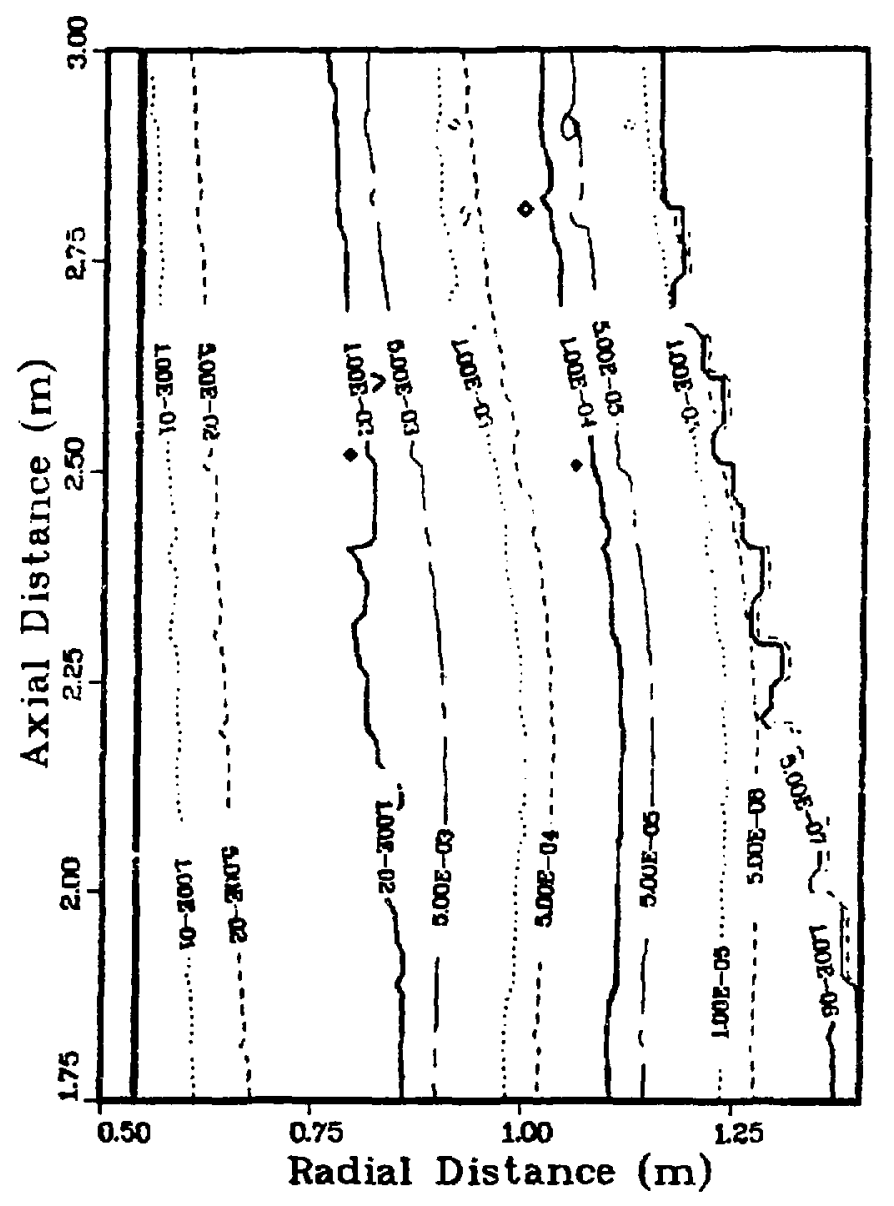

Fig. 45. TRIDENT-CTR gamma-ray heating $\left(\mathrm{MW} / \mathrm{m}^{3}\right)$ at the NBI-duct shutter. See Fig. 26 for geometric model. 


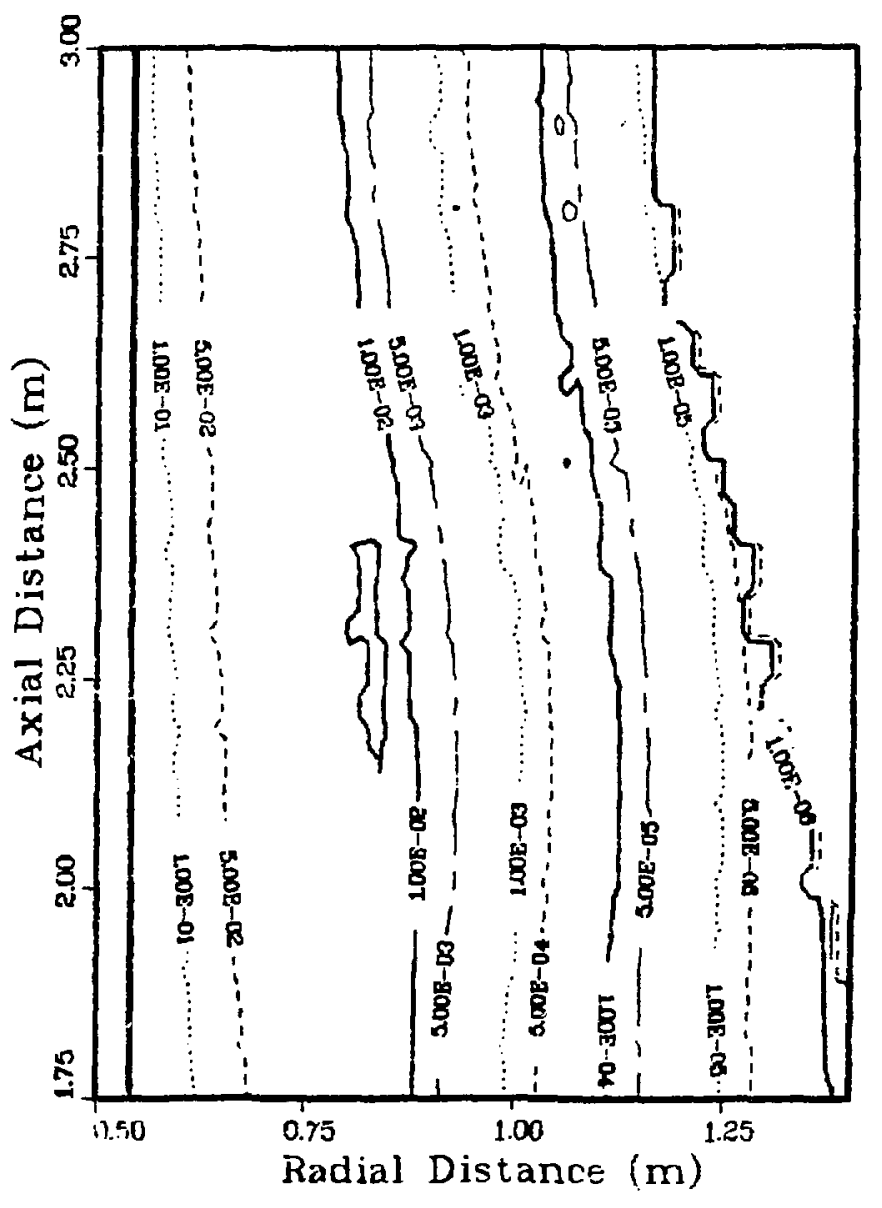

Fig. 46. TRIDENT-CTR total (neutron plus gamma-ray) heating $\left(M W / m^{3}\right)$ at the NBI-duct shutter. See Fig. 26 for geometric model. 
The reader is reminded that the neutron flux values in the duct do not include either the tracklength for neutrons prior to their first incidence on the duct wall, or the tracklength for neutrons which travel in the duct from the plasma chamber to the NBI chamber without having an interaction with the duct shielding. The reason for this is that the generation of the internal boundary source was based on only those neutrons incident on the duct walls; therefore. the calculation precludes the two contributions cited above to the flux in the duct. In addition, when examining these curves, the stepped boundary on the right hand side of the contour plots (i.e., outside boundary of the NBI shield) is the result of overlaying the tapered shield boundary of the TRIDENT-CTR model. with a rectangular plotting grid. Finally, the contour plots are not intended to provide detailed quantitative information but rather a qualitative overview. However, such detailed information is avajlable from the TRIDENT-CTR output files. The total neutron flux contour plot of Fig. 39 provides an overview of the neutron population throughout the NBI shield, whereas a blowup of the flux in the region of the shutter shield is contained in Fig. 40. From these figures it is seen that the NBI shield provides an attenuation of the total flux that varies from $\sim 10^{7}$ near the torus outboard shield to $\sim 10^{4}$ at the end of the duct. Attenuation through the torus outboard shield is about eight orders of magnitude.

Contour plots of the neutron, gamma-ray and total heating as shown in Figs. 39 through 43, respectively, and their corresponding blow-ups in the vicinity of the shutter shield, Figs. 44 through 46 , are self-explanatory. The predominant contributors to the total heating are the gamma rays. Neutron heating at the shutter suield as shown in Fig. 44 illustrates the maxima caused when the neutrons, which have been degraded in energy through scattering in the steel, transport from the steel into a mixture of steel and borated water.

Routines for using TRIDENT-CTR data to generate 30-color contour plots have been developed. Such color contour plots were made for the NBI-duct results but could not be incorporated into this report because of color reproduction limitations. It has been our experience that color contour plots are considerably easier to evaluate on a qualitative and quantitative basis than black and white contour plots.

3. Comparison of MCNP and TRIDENT-CTR. A comparison of the NBI results using the hybrid MCNP/TRIDENT-CTR procedure and the all-MCNP procedure was made 
to provide a means of evaluating the MCNP/TRIDENT-CTR procedure. This comparison is not, however, simply a comparison of two different calculational methods but also includes the differences in the geometric models. Before examining the comparison of these NBI results, it is of value to reiterate these differences.

Figures 19 and 26 are the geometric models for the MCNP and TRIDENT-CTR calculations. The MCNP duct and shield are rectangular in cross section, whereas for TRIDENT-CTR they are modeled as cylindrical. The MCNP model includes the VP-duct penetration of the NBI-duct shield, which is not included in the TRIDENTCTR model of the NBI-duct and shield. The model of the torus outboard shield in TRIDENT-CTR was necessarily squared off; i.e., the inboard boundary of the outboard torus shield was perpendicular to the duct centerline, whereas in the MCNP model it is modeled in accordance with the actual conceptual design. The duct shield thicknesses were stepped in the MCNP model and tapered (more realistic) in the TRIDENT-CTR model. The locations for comparison were chosen to minimize the effect of these differences in shield configurations.

Differences in the calculational procedures include not only the differences in the numerical methods used, Monte Carlo versus discrete ordinates, but also differences in the source treatment and the use of different cross-section sets. In particular, the internal boundary source along the duct wall as used in TRIDENT-CTR was an average value for the four walls of the rectangular duct.

Hence, the comparison which follows does not just provide an evaluation of different numerical techniques (Monte Carlo versus discrete ordinates), but rather of two different approaches for solving a complex three-dimensional problem. As a result, the comparison provides a means of evaluating the hybrid MCNP/TRIDENT-CTR method and its applicaton in a full-scale design problem.

The comparison of the hybrid MCNP/TRIDENT-CTR calculational results to those from the all-MCNP calculations are presented in Table VII. Locations for the regions used in this comparison are identified in Fig. 19. In general, the comparison between the results for the two methods is quite good. The region III results are somewhat different from the others in that the TRIDENTCTR flux is lower and the heating is much higher than the corresponding MCNP values. At present, a quantitative explanation for this anomaly is not available. It is believed to be, in part, a result of several factors, including the perturbation introduced by the vacuum duct penetration, which is contained in the MCNP model and not in the TRIDENT-CTR model, geometric differences 
TABLE VII

MCNP/TRIDENT-CTR NBI-DUCT COMPARISONS

\begin{tabular}{|c|c|c|c|c|}
\hline Region $^{a}$ & Quantity & MCNP & TRIDENT - CTR & $\frac{\text { TRIDENT -CTR }}{\text { MCNP }}$ \\
\hline \multirow[t]{2}{*}{ I } & Flux & $3.26+13(0.0152)^{d}$ & $4.19+13^{e}$ & 1.29 \\
\hline & Heating ${ }^{c}$ & $3.60-1 \quad(0.0249)$ & $3.43-1$ & 0.96 \\
\hline \multirow[t]{2}{*}{ I I } & Flux & $1.46+12(0.0571)$ & $1.83+12$ & 1.25 \\
\hline & Heating & $2.19-2(0.1040)$ & $2.02-2$ & 0.92 \\
\hline \multirow[t]{2}{*}{ I I I } & Flux & $1.58+9(0.0892)$ & $1.54+9$ & 0.97 \\
\hline & Heating & $8.80-5(0.0876)$ & $1.46-4$ & 1.66 \\
\hline IV & Flux $\mathrm{f}^{\mathrm{f}}$ & $5.45+12(0.0653)$ & $5.78+12$ & 1.06 \\
\hline
\end{tabular}

${ }^{a}$ See Fig. 19 for region logation.
$b_{\text {Neutron flux, neutrons } / \mathrm{cm}^{2} \mathrm{~s} \text {. }}$

Neutron plus gamma-ray heating, $\mathrm{MW} / \mathrm{m}^{3}$.

Fractional error.

$\mathrm{e}^{4.19}+13=4.19 \times 10^{13}$.

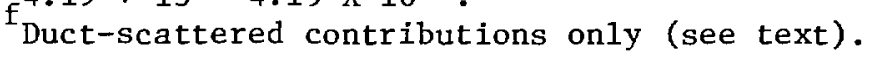

between the cylindrical and rectangular shield models, and differences in the cross sections (e.g., self-shielding effects) used by the two codes. The gamma rays produced in the torus shield have little impact on the NBI shielding, as evidenced by the fact that in region I these gamma rays contribute only $\sim 8 \%$ of the total heating and are a negligible contributor to the region II total heating. The region IV flux given in Table VII is not the same as that shown in Fig. 32. The flux values shown in Fig. 32 are total fluxes according to the normal definition, whereas the region IV values are not total fluxes in either case. The TRIDENT-CTR neutron flux values in the duct do not include either the tracklength for neutrons prior to their first incidence on the duct wall, or the tracklength for neutrons which travel in the duct from the plasma chamber to the NBI chamber without having an interaction with the duct shielding. Thus, the comparable MCNP values for region IV were computed in a consistent manner to TRIDENT-CTR. 
B. VP Duct

1. MCNP Results. Results of the VP-duct MCNP calculations are presented in Fig. 47 through 52. Data are presented concerning the flux in the VP duct and the flow of radiation from the VP duct into the VP chamber. A discussion of these figures follows.

The decrease in the neutron flux as a function of the distance from the duct mouth is shown in Fig. 47. Included on this figure is the contribution to the Lotal flux arising from neutrons direct from the NBI duct that do not interact with the VP-duct walls. The difference between the two curves is the contribution from neutrons that have scattered from the duct shielding. The variation of the scattered contribution to the flux as a function of the distance from the duct mouth is similar to that observed for the NBI duct; i.e., Fig. 32. Fractional errors for the total fluxes are less than $3 \%$ and those for the flux contributions direct from the NBI duct are less than $6 \%$. A combination of the neutron flux falloffs in both ducts is provided in Fig. 48. For this figure, the flux in the VP duct starts at the middle of the NBI-duct segment where the two ducts interface. What appears to be a discontinuity in the fluxes at the intersection of the two ducts, i.e., at $\sim 5.1 \mathrm{~m}$, results from the fluxes being volume-averaged values. The flux falloff down both ducts is very similar; i.e., on the average in the NBI duct the flux decreases by a factor of $\sim 10.6$ per metre, whereas in the VP duct it decreases by $\sim 8.4$ per metre. Errors for the data on this figure are less than $3 \%$.

A comparison of the neutron flux spectra over the first $0.3 \mathrm{~m}$ and last $0.1 \mathrm{~m}$ of the VP duct is provided by Fig. 49. The spectrum at the end of the duct is significantly softer than the spectrum at the entrance to the duct. The spectrum at the end of the duct contains a very small high-energy component, which is to be expected because the only way plasma neutrons can arrive at this location is through multiple scattering that degrades their energies. Total error of the spectrum at the duct mouth is less than $1 \%$ and the individual energy bin errors are all less than $8 \%$. At the end of the duct, the total error is less than $2 \%$ and the individual values are less than $10 \%$ for energies less than $0.823 \mathrm{MeV}$. Above $0.823 \mathrm{MeV}$ the values are statistically suspect but are very small contributors to the total flux. 


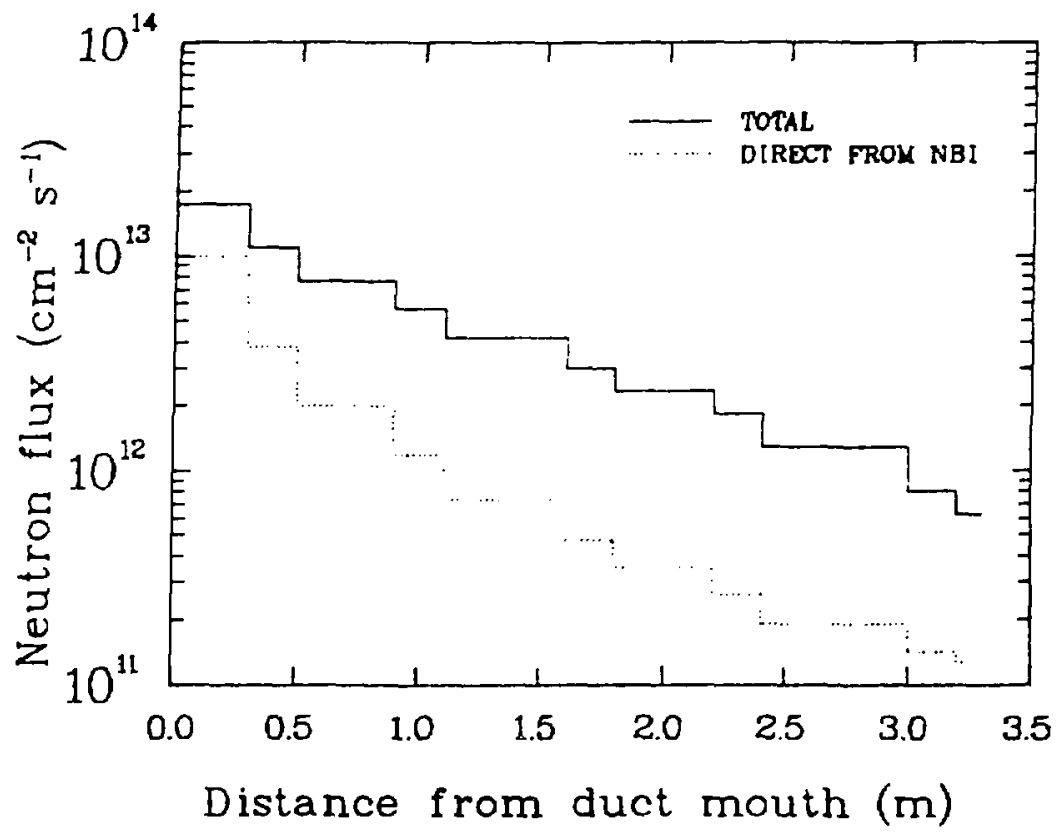

Fig. 47. Comparison of the total neutron flux down the VP duct to the flux from the first flight in the VP duct of neutrons direct from the NBI duct.

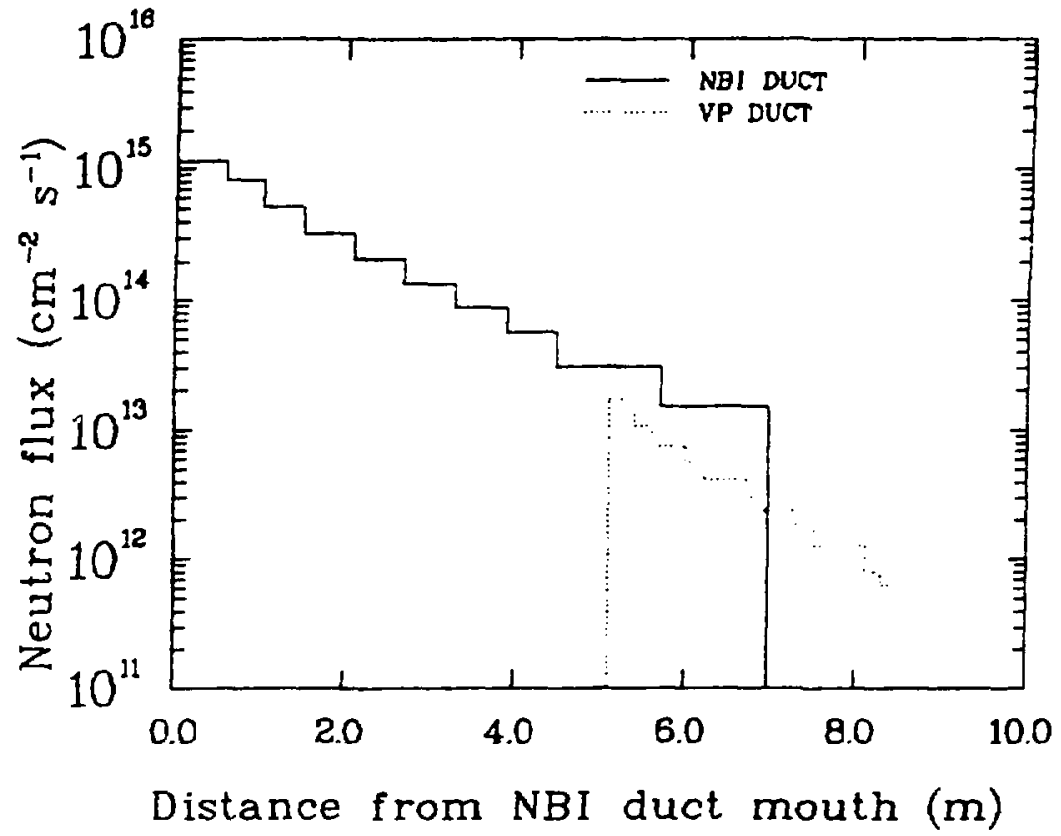

Fig. 48. Neutron flux as a function of the distance from the NBI-duct mouth for both the NBI and VP ducts. 


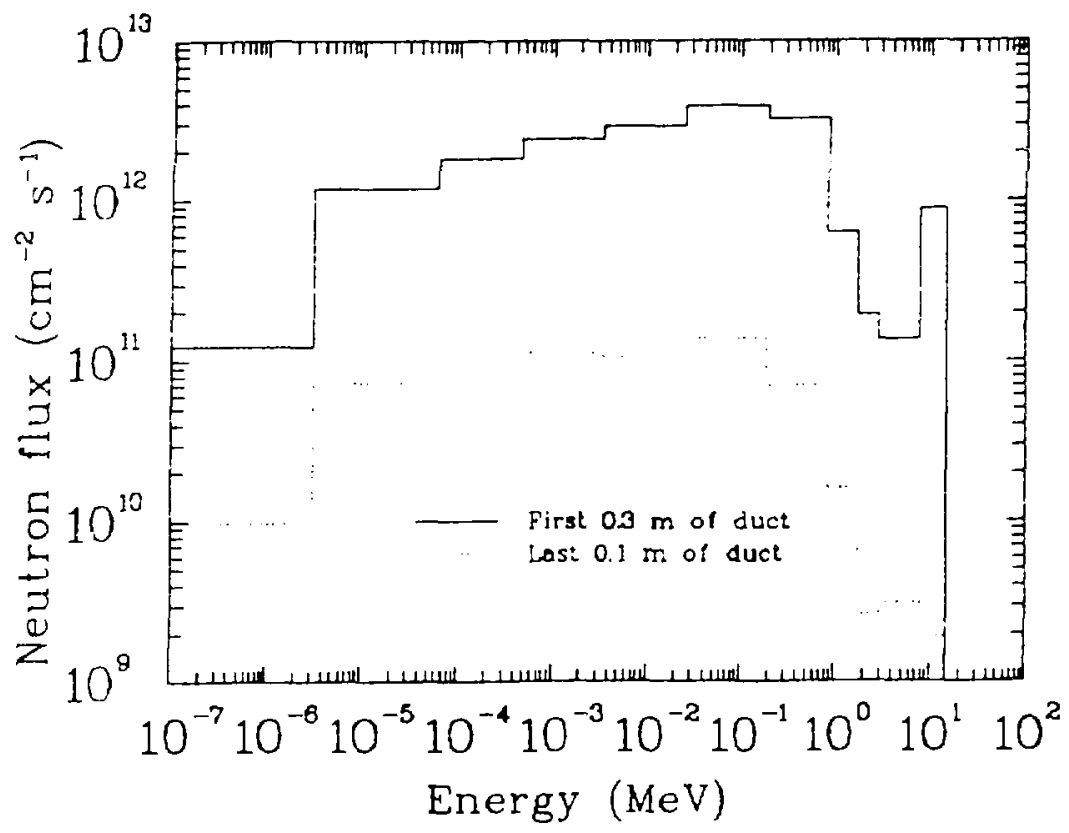

Fig. 49. Comparison of neutron flux spectra at the beginning and end of the VP duct.

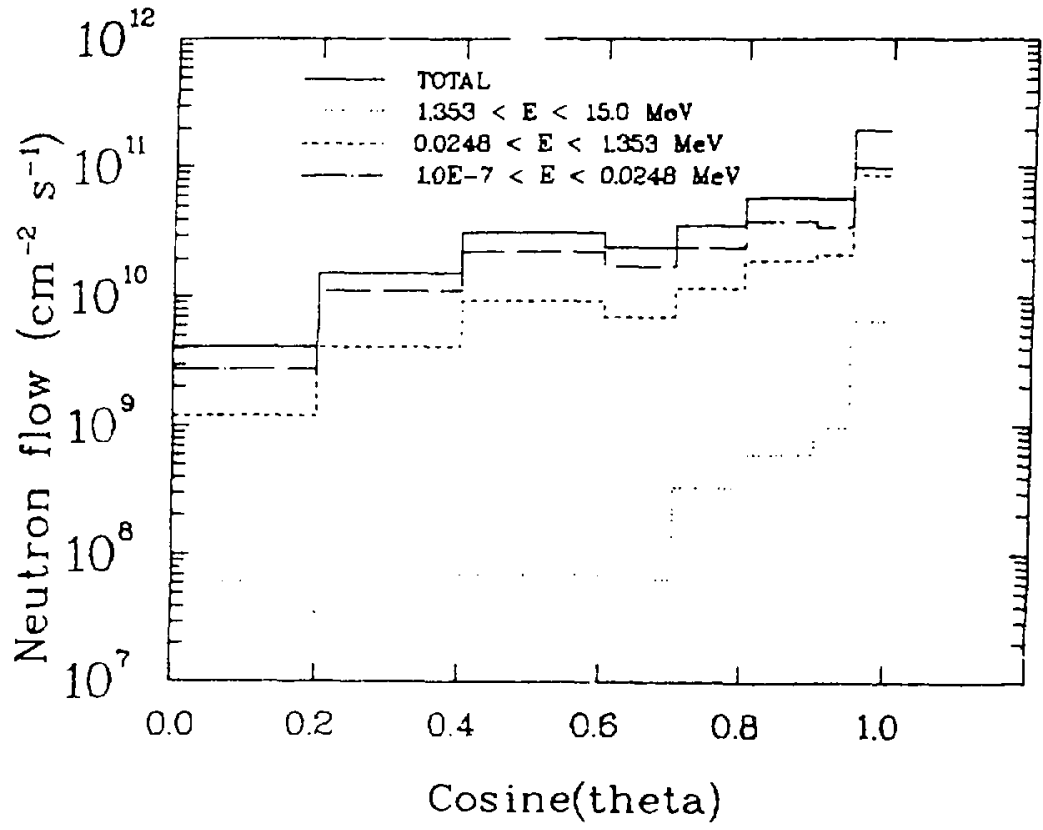

Fig. 50. Energy-dependent, angular distribution of neutrons entering the VP chamber from the VP duct. Theta is the angle between the neutron direction and the duct axis; i.e., a polar angle. 


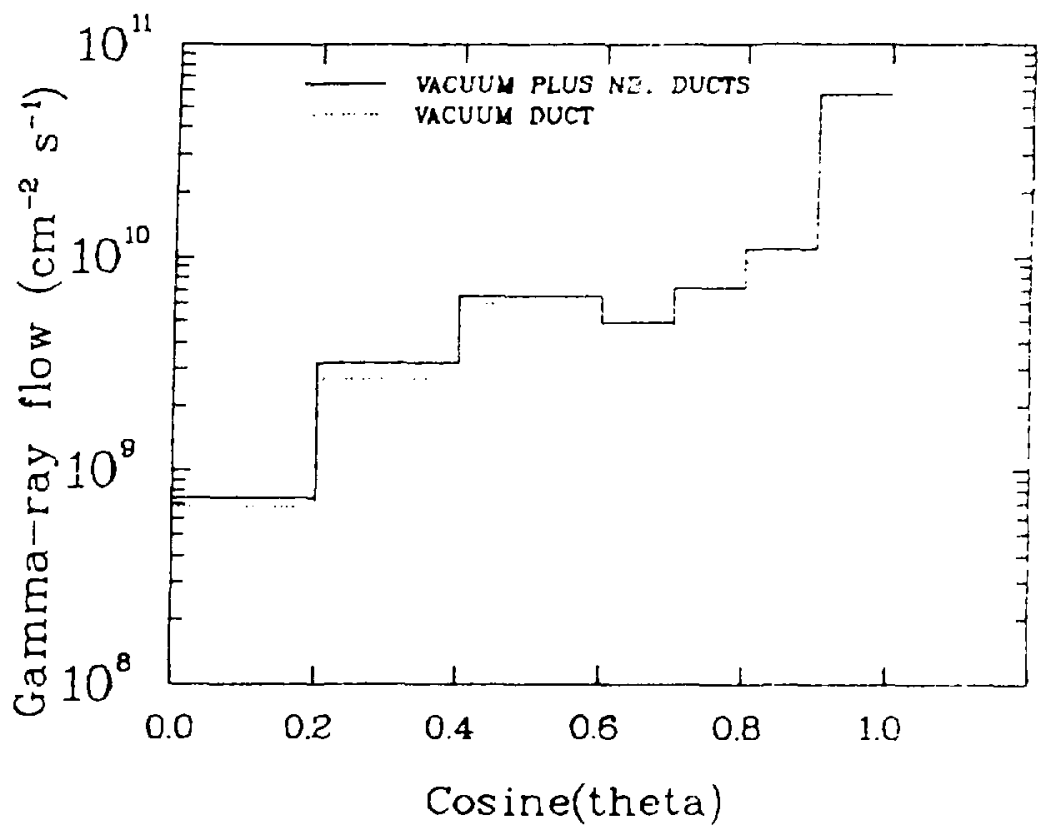

Fig. 51. Angular distribution of gamma ray flowing from the VP duct into the VP chamber and the sum of those originating in both the NBI- and VP-duct shields.

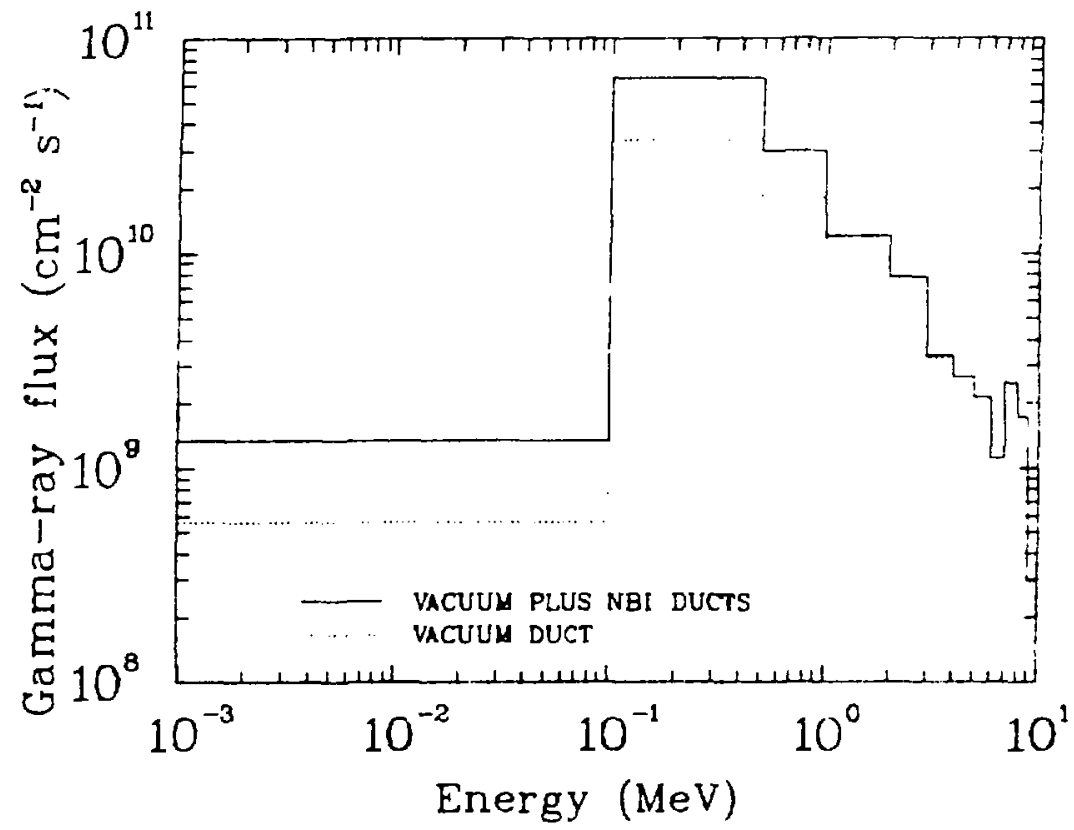

Fig. 52. Energy-spectrum of gamma-ray flux in the last $0.1 \mathrm{~m}$ of the VP duct for gamma rays originating in $l$-duct shield, and the sum of those orig nating in the NBI- and VP-duct shields. 
Figure 50 is a plot of the energy-dependent angular distribution of neutrons flowing into the VP chamber from the VP duct. These histograms are similar to those for the neutrons entering the NBI chamber except that the angular distributions are not as highly peaked into the VP chamber. Again, it is observed that the higher the energy the more strongly peaked is the angular distribution, and also that the flow of neutrons is dominated by those with low energies. Errors for the total flux angular distribution are all less than $5 \%$, except for the first interval where it is $8 \%$. Errors for the two lowest energy curves are, in general, less than $10 \%$ except for the first two cosine bins, where the errors are slightly greater than $10 \%$. The highest energy curve is statistically less reliable.

The angular distribution of the gamma-ray flow from the VP duct into the VP chamber is presented in Fig. 51. This distribution is characteristically forward peaked and it can be seen that the gamma rays originating in the VP-duct shield are predominant contributors to the flow. As expected, gamma rays originating in the NBI duct contribute, with any significance, only to the largest cosine bin, and in fact dominate the contribution in that bin. However, because the error associated with this contribution is $16 \%$, it is less reliable and the total for the two ducts is likewise less reliable for this cosine bin. With the exception of the lowest cosine bin, the errors associated with the VPduct curve are a11 less than $10 \%$.

The average gamma-ray flux spectrum in the last 0.1 metre of the duct is presented in Fig. 52. Again, the spectra for the gamma rays originating in the VP-duct shield and those originating in both the VP-duct and NBI-duct shield are shown. For the VP duct the data have errors of 1ess than $10 \%$ for energies below $2.0 \mathrm{MeV}$, but above this energy they become progressively larger and thus statistically unreliable.

2. TRIDENT-CTR Results. Contour plots of the neutron flux, neutron heating, gamma-ray heating and, total heating are presented in Figs. 53 through 56, respectively. The geometric model corresponding to these results is shown in Fig. 27. Recall from Section IV.B that this calculation is a conservative representative of the "hot" wall of the VP duct. The ordinate on these curves extends from 0.0 to 3.3 metres with the VP-duct mouth at 3.3 metres and the end of thr: VP duct at 0.0 . 


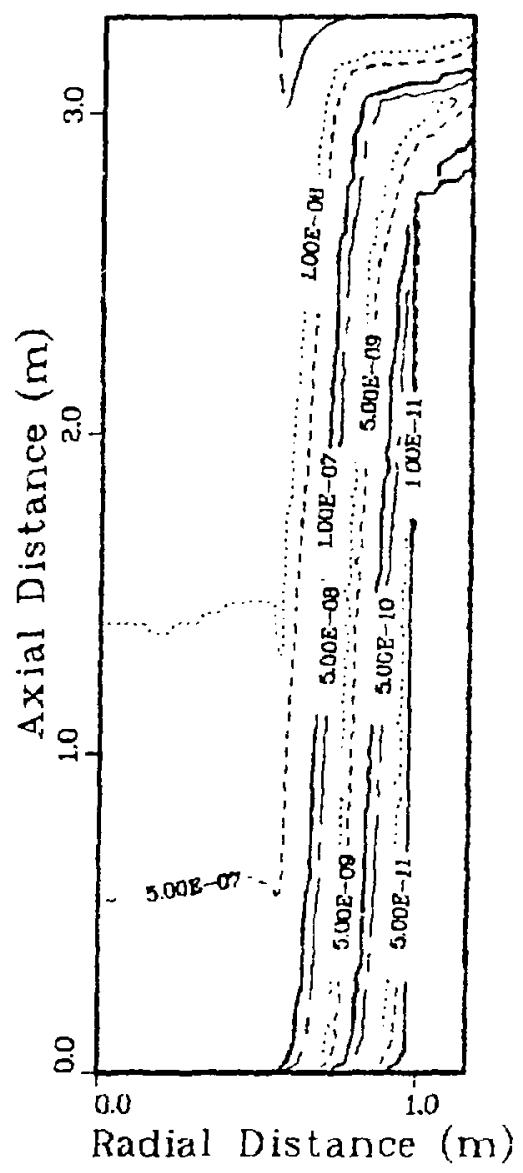

Fig. 53. TRIDENT-CTR total neutron flux $\left(\mathrm{cm}^{-2} \mathrm{~s}^{-1}\right)$ in the VP-duct shield. Multiply values by $5.05 \times 10^{17}$. See Fig. 27 for
geometric mociel. 


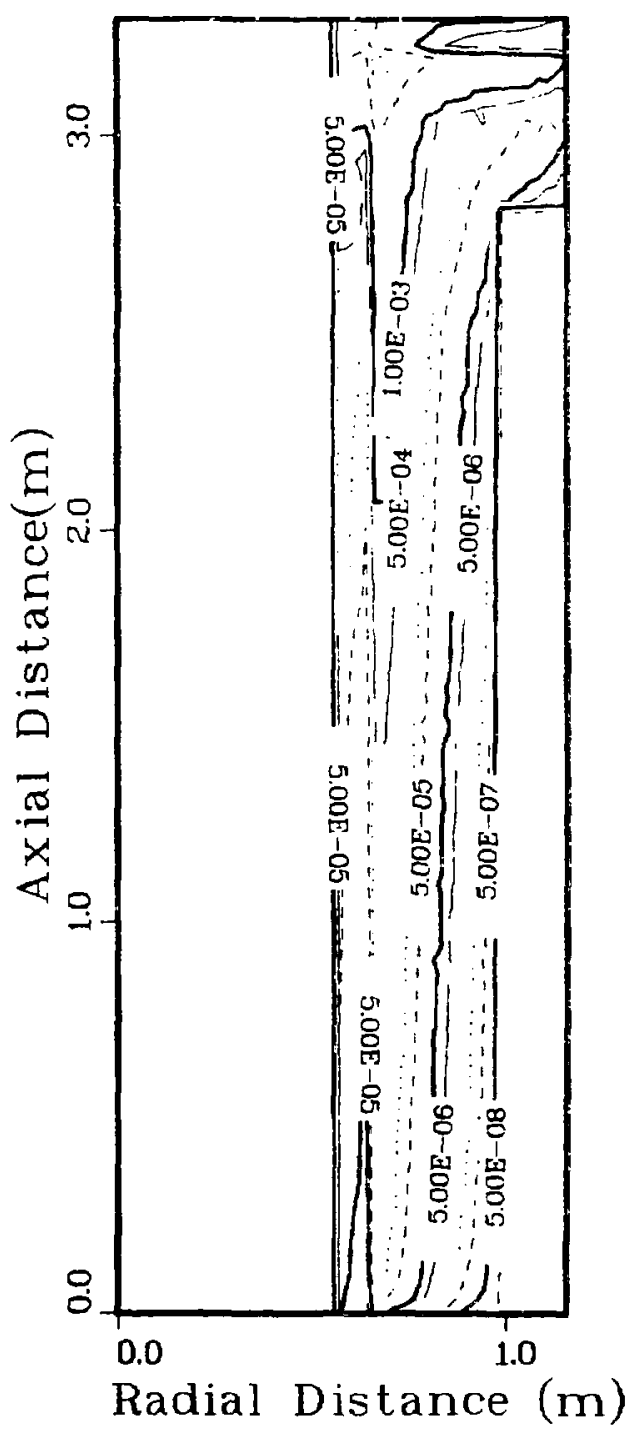

Fig. 54. TRIDENT-CTR neutron heating $\left(\mathrm{MW} / \mathrm{m}^{3}\right)$ in the VP-duct shield. See Fig. 27 for geometric model. 


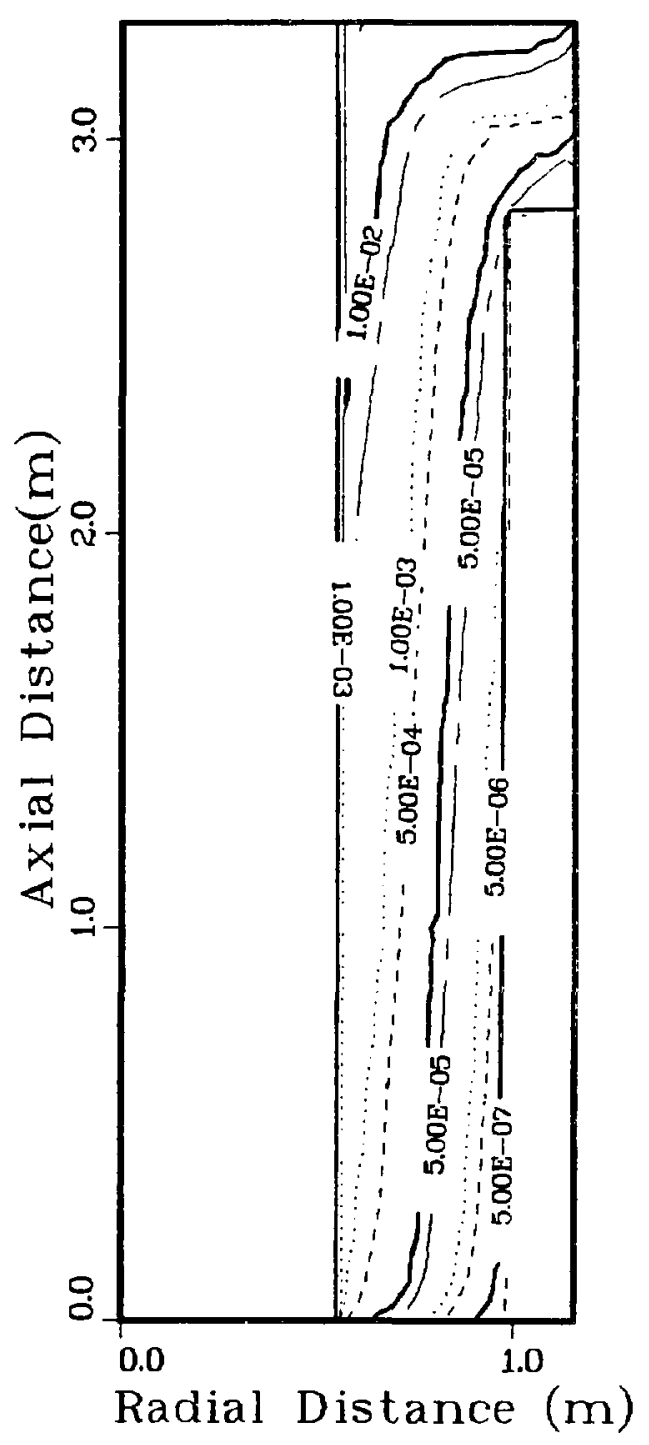

Fig. 55. TRIDENT-CTR gamma-ray heating $\left(\mathrm{MW} / \mathrm{m}^{3}\right)$ in the VP-duct shield. See Fig. 27 for geometric model. 


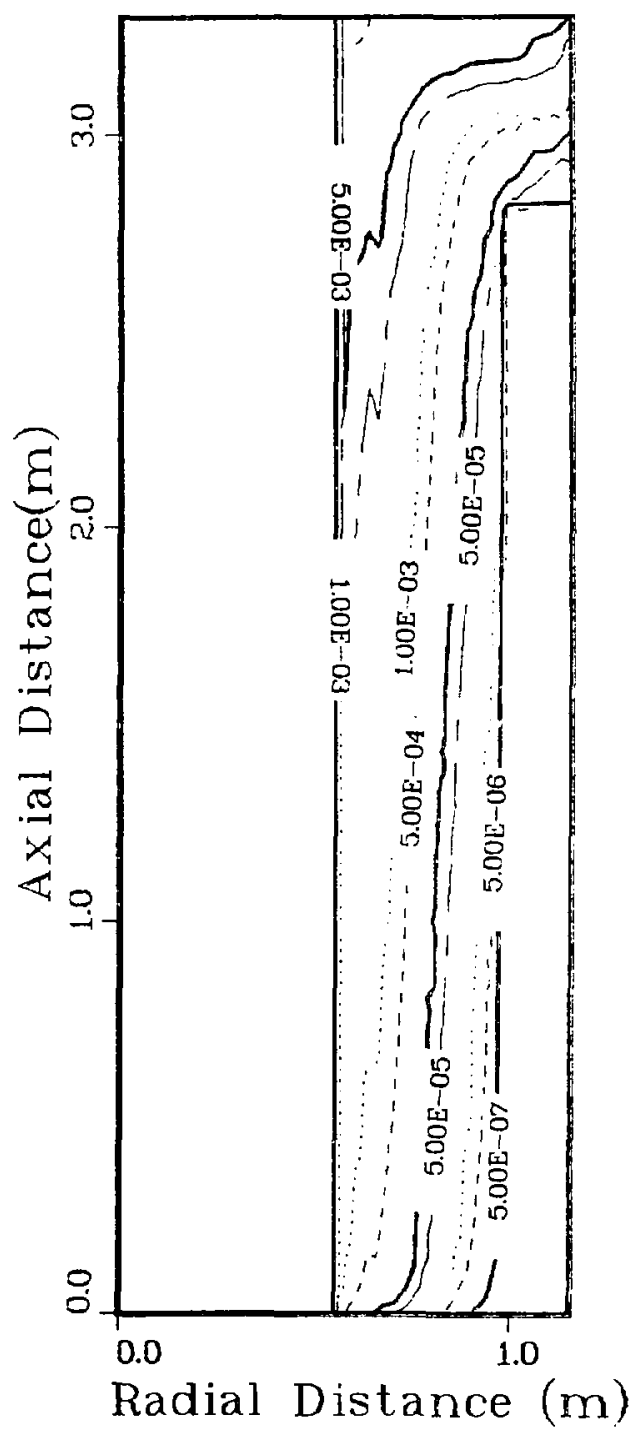

Fig. 56. TRIDEATT-CTR total (neutron plus gamma-ray) heating $\left(\mathrm{MW} / \mathrm{m}^{3}\right)$ in the VP-duct shield. See Fig. $27 \mathrm{f} \cdot \mathrm{r}$ geometric model. 
These contour plots are intended to present a qualitative overview of the flux and heating in the VP-duct shield. Also, the neutron flux values in the duct do not include either the tracklength for neutrons prior to their first incidence on the duct wall, or the tracklength for neutrons that travel in the duct from the NBI duct to the VP chamber without having an interaction with the VP-duct shielding. The reason for this is that the generation of the internal boundary source was based on only those neutrons incident on the duct walls and therefore the calculation precludes the above two cited contributions to the flux in the duct. The contour plots are generally straightforward and self-explanatory. Detailed values from the TRIDENT-CTR output files are available from the authors upon request.

3. Comparison of MCNP and TRIDENT-CTR Results. Section III.A.3 contained a discussion of the comparison of the MCNP results with those from the hybrid MCNP/TRIDENT-CTR calculation for the NBI duct. This section provides a similar comparison for the VP duct. In that previous section the implications of the comparison were discussed and thus will not be repeated here, even though most. of them are applicable to the VP duct as well. However, the difference in the sources used for the VP-duct MCNP and MCNP/TRIDENT-CTR calculations are significantly different and are discussed below.

The VP-duct TRIDENT-CTR internal boundary source was not an average for the four duct walls. Rather, it was based on the energy spectrum and angular distribution of neutrons incident on duct wall 2 (see Fig. 20), which is the "hot" wall. This approach was taken because it would ensure conservatism of the calculation by considering the most intense source. This source was subsequently renormalized to represent the maximum intensity on the rectangular duct wall, but in so doing it results in a total incidence on the cylindrical wall that is $\sim 2.1$ times greater than that which is incident on the four rectangular walls in the MCNP model. Furthermore, there is a significant difference between the incident energy spectrum of the TRIDENT-CTR calculation and that incident on the four walls of the MCNP calculation. In particular, only the "hot" wall has a significant incident high-energy component of neutrons (see Fig. 24.) The net result is tiat in the TRIDENT-CTR calculation $\sim 13 \%$ of the neutrons incident on the duct walls have energies in the range 13.5 to $15.0 \mathrm{MeV}$, while for the MCNP calculation only $\sim 6 \%$ are in this energy range. 
Further conservatism is included in the TRIDENT-CTR boundary source at the lip of the VP duct. This TRIDENT-CTR source is azimuthally symmetric in intensity and angular distribution about the VP-duct axis, whereas in reality the source is severely truncated on two sides by the NBI-duct shield walls. Furthermore, a most conservative angular distribution was used, as discussed in Section IV.B.

From the foregoing it would appear that the TRIDENT-CTR results should be larger than the corresponding MCNP results. However, it should be emphasized that the internal boundary source on the duct cylindrical wall was correct in intensity for the "hot" wall and therefore the conservatism, to a large part, of the calculated quantities results from the correlation of one part of the wall with the rest of the wall; e.g., through the scattering of neutrons back into the duct. With TRIDENT-CTR this correlation is most probably too strong as a result of the source used.

Comparison of the MCNP results with those from the hybrid MCNP/TRIDENT-CTR calculation are presented in Table VIII. The hybrid MCNP/TRIDENT-CTR method results are referred to as TRIDENT-CTR results. The regions used for the comparison are indicated in Fig. 25. Regions VI, VII, X, and XI are approximately the same width as the duct "hot" wall; i.e., $0.8 \mathrm{~m}$. In general, the MCNP neutron fluxes and total (neutron plus gamma-ray) heating are lower than those from the TRIDENT-CTR calculation, as would be expected because of the reasons previously cited. Regions VI, VIII and XI all border the outside of the shield and have TRIDENT-CTR to MCNP flux ratios that are fairly constant; i.e., they vary from 1.52 to 1.67 . Further detailed edits were made for Region VI to determine the longitudinal variation in the flux for this region, as indicated in Fig. 57, which is a cross-sectional view through the duct at the axial location of Region VI. There appears to be a significant variation in the flux, suggesting that agreement between the MCNP and TRIDENT-CTR results is strongly dependent on the size of the MCNP edit region. Evaluation of this inference would require subdividing Region VI into even smaller regions than shown in Fig. 57, and, of course, running many more particle histories to obtain reasonable statistics.

Although Regions VI and VIII have flux ratios that are nearly the same, the ratio increases when Regions IX, $X$ and $X I$ are considered. The Region IX values are the ratio of fluxes that do not include either the tracklength for neutrons prior to their first incidence on the duct wall, or the track- 


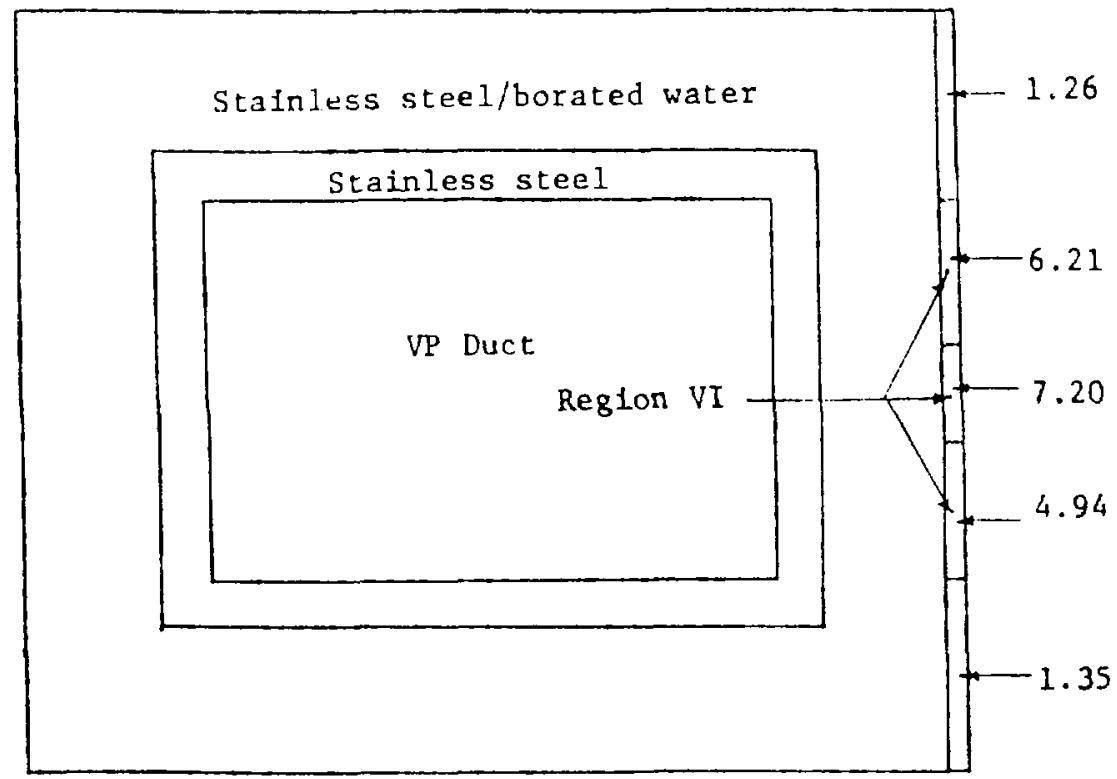

Fig. 57. Cross section of the MCNP, VP-duct model at the axial location of Region VI (see Fig. 25.) Numbers to the right are volume averaged neutron fluxes times $10^{-9}$.

length for neutrons which travel in the duct from the NBI duct to the VP chamber without having an interaction with the VP-duct shielding. These modified fluxes were compared in the duct because this is the quantity calculated by TRIDENTCTR in the duct. This flux ratio, however, is the largest ratio quoted in Table VIII. In general, we note the flux ratio decreases toward unity with distance from the duct into the shield. At large distances into the shield, the neutron population is strongly dependent on the high-energy component of the neutrons incident on the shield wall. Therefore, at locations deep within the shield, the flux will be weakly dependent on the backscatter from the other walls of the duct as such scattering will decrease their energy such that they will be more strongly attenuated. On this basis one could expect, if all other things were equal, that the TRIDENT-CTR to MCNP flux ratio would converge toward unity deep within the shield as the data of Table VIII appears to indicate. 
MCNP AND TRIDENT-CTR VP-DUCT COMPARISONS

\begin{tabular}{|c|c|c|c|c|}
\hline \multirow{2}{*}{ Region ${ }^{a}$} & \multirow{3}{*}{$\begin{array}{l}\text { Quantity } \\
\text { Flux }\end{array}$} & \multirow{3}{*}{$\begin{array}{l}\text { MCNP } \\
5.98+9(0.0741)^{d}\end{array}$} & \multicolumn{2}{|c|}{ TRIDENT-C' } \\
\hline & & & TRIDENT - CTR & MCNP \\
\hline \multirow[t]{2}{*}{ VI } & & & $9.11+9^{e}$ & 1.52 \\
\hline & Heating ${ }^{c}$ & $2.87-4(0.0572)$ & $5.83-4$ & 2.03 \\
\hline \multirow[t]{2}{*}{ VI I } & Flux & $2.64+12(0.0156)$ & $2.29+12$ & 0.87 \\
\hline & Heating & $2.77-2(0.0207)$ & $2.30-2$ & 0.83 \\
\hline \multirow[t]{2}{*}{ VIII } & Flux & $9.58+8(0.1427)$ & $1.48+9$ & 1.54 \\
\hline & Hearing & $6.58-5 \quad(0.0893)$ & $1.44-4$ & 2.19 \\
\hline IX & $\operatorname{Flux} f$ & $5.00+11(0.0268)$ & $1.17+12$ & 2.34 \\
\hline \multirow[t]{2}{*}{$\mathrm{X}$} & Flux & $2.22+11(0.0646)$ & $4.38+11$ & 1.97 \\
\hline & Heating & $1.96-3(0.0873)$ & $4.87-3$ & 2.48 \\
\hline \multirow[t]{2}{*}{$X I$} & Flux & $8.25+9(0.0936)$ & $1.38+10$ & 1.67 \\
\hline & Heating & $1.98-4(0.0811)$ & $4.05-4$ & 2.05 \\
\hline \multicolumn{5}{|c|}{$\begin{array}{l}a_{\text {See Fig. }} 25 \text { for region location. } \\
b_{\text {Neutron flux, neutrons } / \mathrm{cm}^{2} \mathrm{~s} .} \\
c_{\text {Neutron plus gamma-ray heating, } \mathrm{MW} / \mathrm{m}^{3} \text {. }} \text {. } \\
d_{\text {Fractional error. }} \\
e_{9} 1+9=9.11 \times 10^{9} .\end{array}$} \\
\hline
\end{tabular}


Examination of Table VIII reveals that Region VII is anomalous with respect to the other data. In particular, the flux and heating ratios indicate that MCNP is slightly higher than TRIDENT-CTR for this region, which is the opposite of the case at each of the other regiors. A detailed examination of both the MCNP and TRIDENT-CTR data was made, but all data examined appeared internally consistent and also consistent with the physics of the problem. All explanations for this anomaly so far investigated are not compatible with the remaining data. Because of the limited information obtained from the MCNP calculations, it has not been possible to isolate the scurce of this anomaly.

A comparison was also made between the MCNP and TRIDENT-CTR neutron fluxes in the duct, as a function of distance from the VP-duct mouth. These fluxes, like those reported for Region IX in Table VIII, do not include the tracklength for neutrons prior to their first incjdence on the duct wall, or the tracklength for neutrons that travel in the VP duct from the NBI duct to the VP chamber without having an interaction with the duct shielding. The ratio of these TRIDENT-CTR fluxes to the corresponding MCNP fluxes was essentially constant over the length of the duct; i.e., the ratios varied from 2.24 to 2.39 and the error associated with the MCNP values was less than $3 \%$. These ratios are consistent with those reported for Region IX in Table VIII, and further illustrate the conservatism introduced by the TRIDENT-CTR source as previously discussed.

\section{Comments on Accuracy.}

Descriptions of the calculations made using the purely Monte Carlo approach and the hybrid Monte Carlo/discrete-ordinates method have been presented together with the results obtained. Two central questions relate to how good the results are in an absolute sense, and how meaningful are the comparisons between the two methods.

Ideally, one could list all the approximations made in geometric modeling, numerical methods, cross sections, etc., and the per cent error introduced in the various problem results by each. However, such an analysis is far beyond the scope of this report. What follows is a discussion to provide the reader a "feel" for the accuracy of the results and the meaning of the comparisons.

The question of accuracy can be considered in relation to the 3-D Monte Carlo results because they were taken as the standard values for this effort. The accuracy of the Monte Carlo results can only be discussed in terms of a particular geometric configuration, plasma source, and shield design. Thus, how 
well the results for the conceptial design used here agree with, for example, those for some future as-built design is irrelevant; knowledge of this agreement is, of course, important to the ETF effort, but not in determining the accuracy of the Monte Carlo results.

There are three major factors that have an impact on the Monte Carlo results: (a) the use of a uniformly distributed plasma source, (b) homogenization of the laminated shields, and (c) the cross sections. The radial plasma distribution is certainly not uniform, and the plasma probably also has poloidal and toroidal variation, especially near the NBI's. The neutrons entering the NBI duct can be divided into two classes; i.e., a high-energy component (unscattered) and a low-energy component (scattered). The scattered component is likely to be only slightly affected by toroidal and radial variations, and possibly highly affected by large poloidal variations. The highenergy component, however, could be strongly affected by variations in any of the three spatial parameters. One can easily visualize exotic plasma distributions which could cause a severe departure from the results that were obtained with a uniform distribution. However, within the framework of reasonable plasma distributions, it is estimated that the plasma distribution would not result in more than a factor of two difference in the results. Given a reasonably well-defined plasma distribution, it could be modeled in 3-D, thereby eliminating, for the most part, this error.

Treatment of the laminated shields as homogeneous slabs is probably the least of the three approximations except insofar as the ratio of metal to water may vary throughout the laminated shield. However, it could possibly have a significant effect on the heating in the shields immediately adjacent to the ducts, which were taken to be solid steel. The neutron flux and total heating deep within the shields are probably not highly sensitive to this approximation and it is estimated that the error introduced is probably not more than a factor of 1.5. In any event, this is another removable error in that the zone homogenization could be refined.

Error introduced through the cross sections is not easily quantified either because it is dependent on space, energy, and the response function of interest. However, based upon other uncertainty analyses, ${ }^{11}$ it is not unrealistic to estimate a factor of two as a standard uncertainty (i.e., standard deviation). It is worth noting that the uncertainty caused by the variances 
and covariances of the cross sections and secondary emission spectra is irremovable tor practical purposes; i.e., removal would require a massive technological effort of measurement, evaluation, and processing, or expensive ad hoc integral experiments. Also, this error is equally manifested and correlated in direction in both our calculation methods insofar as the same evaluated data (ENDF-B/IV and $V$ ) are, in general, used for deterministic and stochastic transport calculations.

An attempt to combine these uncertainties using a simplistic quadraticaverage or root-mean-square approach is inappropriate because the uncertainties themselves are not entirely independent. Furthermore, the overall uncertainty is a function of space, energy and the response of interest. Under these conditions, our best "guess" is that the Monte Carlo results are within a factor of a few of those for the design configuration we considered. This should not be interpreted to mean that the Monte Carlo results are too high or too low by that factor but rather that, with a nonuniform plasma distribution and distinct shield laminations, the results could differ by that much.

The comparison between the hybrid method and the MCNP results is not simply a comparison between iumerical methods (combination stochastic-deterministic versus stochastic). Rather, it is a comparison between the application of two different (numerical, dimensionality, modeling, data) calculational methods to the same design configuration. Thus, the comparison provides a means of evaluating both the numerical methods and their application, but we are not able to distinguish completely whether it is the method or the application which is the cause of differences between the two sets of calculated results. In this context, the comparisons are indeed meaningful. In fact, it is especially valuable to the designer to know how good his overall design procedure is, not for some idealized configuration, but rather when applied to a full-scale 3-D design configuration.

\section{CONCLUSIONS}

The analysis has demonstrated that a marriage of MCNP and TRIDENT-CTR (i.e., Monte Carlo and discrete ordinates) can be effectively used in full-scale design applications for the analysis of large duct shields. The validation of 
this procedure was provided through comparison with selected 3-D Monte Carlo results. Although currently existing Monte Carlo and discrete-ordinates codes were used in the malysis, special purpose rutines were written to facilitiate linkage of the tho codes. Codes for linking Monte Carlo and discrete ordinates are problein dependent; however, it is our upinion that some of the linkage can be generalized and the procejure standardized to the point whereby the method can be appinahle lo a variety of problems without undue difficulty. Total computer costs were found to be a very minor portion of the total effort.

Besides proviling neutron and gamma-ray fluxes and heating rates in the NBl-and VP-cuct shields, this effort has aided the effort to identify potential radiation effects problems in the NBI and VP chambers. In particular, the high neutron fluences entering the NBl chamber, even with the use of the present conceptual shutter shield, may couse serious difficulties.

\section{VIJ REFERENCES}

1. Donald J. Dudziak and P.G. Young, "Review of New Developments in Fusion Nuclcunics," Proc. Pourth ANS Topical Meeting Technol. of Controlled Nucl. Fusion (King uf l'russia, Penn., Oclober 1980), Pp. 14-17.

2. Jos Alamos Ciroup X-6, "MCNP - A General Monte Carlo Code for Neutron and Photon "Transport," Los Alamos National Laboratory report LA-7396-M, Revised (Vovenber 1979).

3. T.J. Seed, "TRIDFN-("l'R liser's Manual," Los Alamos National Laboratory report LA-7835-M, Revised (November 1979).

4. P. D. Soran and R. E. Seamon, "Graphs of the Cross Sections in the Recommended Monte Carlo Crcss-Section Library at the Los Alamos Scientific Laborator; " Los Alamos National. Laboratory report LA-8374-MS (May 1980).

5. H.A. Sandmeier, G.E. Hansen, R.E. Seamon, T. J. Hirons, and A. H. Marshall, "Coupled Neutron-Gamma Multigroup-Multitable Cross Sections for 29 Materials Pertinent to Nuclear Weapons Effect Calculations Generated by LASL/TD Divisiun," Los Alamos National Laboratory report LA-5137 (February 1974).

6. C.I. Baxman and P.G. Young, "Applied Nuclear Data Research and Development January ] - March 31, 1977," Los Alamos National Laboratory report LA-6893-Pti (July 1977) .

7. R. E. MacFarlane and R. J. Bitrett. "TRANSX," Los Alamos National Laboratory Mejnorandum T-2-L-2923 to Discribution, Aug. 24, 1978. 
8. G. I. Bell, .. E. Hansen, and H. A. Sandmeier, "Multitable Treatments nf Anisotropic sattering in $\mathrm{S}_{\mathrm{N}}$ Multigroup Transport Calculations," Nur: Sci. Eng. 28, $376\left(1967^{\circ}\right.$

9. B. G. Carlson, "Tables of Symmetric Equal Wuight Quadrature $\mathrm{EQ}_{N}$ Over the Unit Sphere," Los Alamos National Laboratory report LA-4734 (July 1971).

10. Fred Beranek and R. W. Conn, "Time-Dependent Neutrunics for Laser Fusion Blanket and Materiais Analysis." University of Wisconsin Nucleai Engireering Departinent report UWFDM- $=10$ (June 1977).

11. S. A. W. Gerstl, R. J. LaBauve, and P. G. Young, "A Compchensive Neutron Cross-Section and Secondary Enerğy Distribution Licertainty Analysis for 3 Fusion Reactor," Los Alamos National Laboratory report LA-8333-MS (May 1980). 